\title{
Making Sense of Food Risk Information The Case of Organic Food
}
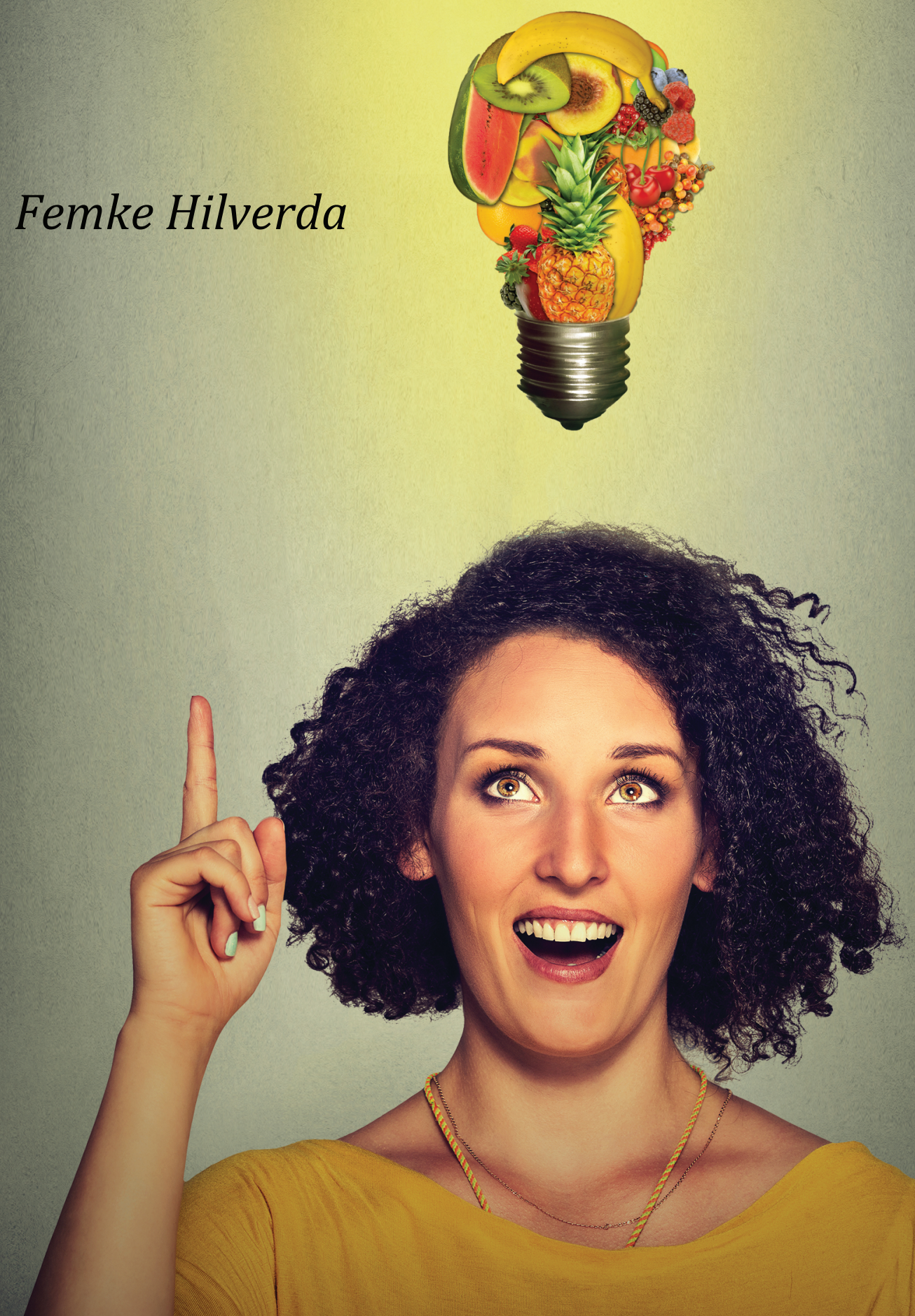


\section{MAKING SENSE OF FOOD RISK INFORMATION:}

The case of organic food

FEMKE HILVERDA 
The research presented in this dissertation was funded by the Netherlands Food and Consumer Product Safety Authority.

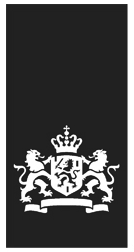

Nederlandse Voedsel- en

Warenautoriteit

Ministerie van Economische Zaken

Cover design: Inga Schwabe

Print and layout: Gildeprint - The Netherlands

ISBN: 978-90-365-4276-0

DOI: $10.3990 / 1.9789036542760$

Thesis, University of Twente, 2017

Copyright (C) 2017 Femke Hilverda, Enschede, the Netherlands.

All rights reserved. No parts of this dissertation may be reproduced or transmitted in any form or by any means without prior permission of the author. 


\title{
MAKING SENSE OF FOOD RISK INFORMATION: \\ THE CASE OF ORGANIC FOOD
}

\author{
PROEFSCHRIFT \\ ter verkrijging van \\ de graad van doctor aan de Universiteit Twente, \\ op gezag van de rector magnificus, \\ Prof. dr. T.T.M. Palstra, \\ volgens besluit van het College voor Promoties \\ in het openbaar te verdedigen \\ op donderdag 23 februari 2017 om 14.45 uur \\ door \\ Marie-Susanne Dieudonnée Hilverda \\ geboren op 6 november 1986 \\ te Breda
}


This dissertation has been approved by the promotor prof. dr. E. Giebels and the copromotor dr. M. Kuttschreuter. 
PROMOTIECOMMISSIE

\section{Promotor}

Prof. dr. E. Giebels

Universiteit Twente

\section{Copromotoren}

Dr. M. Kuttschreuter

Universiteit Twente

\section{Voorzitter}

Prof. dr. Th.A.J. Toonen

Universiteit Twente

\section{Leden}

Prof. dr. B. M. Fennis

Rijksuniversiteit Groningen

Prof. dr. J.E.W.C. van Gemert-Pijnen

Universiteit Twente

Prof. dr. A. Opperhuizen

Universiteit Maastricht

Prof. dr. P.J. Taylor

Lancaster University \& Universiteit Twente

Prof. dr. ir. W. Verbeke

Universiteit Gent 



\section{Table of Contents}

Chapter 1: $\quad$ Introduction 9

Chapter 2: Word associations with "Organic" $\quad 23$

Chapter 3: $\quad$ The effect of online social proof regarding organic food 45

Chapter 4: $\quad$ Social media mediated interaction with peers, experts and anonymous authors 65

Chapter 5: $\quad$ Online information sharing about risks 91

\begin{tabular}{ll} 
Chapter 6: & Discussion \\
\hline
\end{tabular}

References 133

$\begin{array}{lr}\text { Summary } & 147\end{array}$

Samenvatting $\quad 153$

Dankwoord 159

KLI Dissertation Series $\quad 163$ 



\section{CHAPTER 1}

INTRODUCTION 
New information about food issues is in abundance available to individuals, coming from a variety of sources, such as television and newspapers, and also friends and family. Individuals have to make sense of this information to make well-informed decisions about their food intake (Van Dijk, Fischer, \& Frewer, 2011). Sense-making is an important process in a complex society (Colville, Brown, \& Pye, 2012). It involves recognizing a problem, seeking, finding and integrating new information in a way that there is no tension between the newly encountered information and one's own vision and beliefs (Weick, 1995; Weick, Sutcliffe \& Obstfeld, 2005). When making sense of information, individuals do not only use their own observations, but also use the observations of others (Dervin, 1983), and sense-making is likely influenced by these others.

Results of the FP-7 project 'FoodRisC' (Barnett et al., 2011) already showed that social media and online news channels are important during food incidents and that the social context is an important determinant of the individual's response in terms of information seeking behaviour. Building on FoodRisC, my PhD-project aims to gain insight in the way individuals make sense of (organic) food risk information and in the way their (online) social environment shapes the way in which they respond to the available information. As such, it combines insights from (risk) communication science with social psychological theories. Outcomes from this dissertation provide insight in the associations individuals have with respect to eating organic, the way individuals make sense of risk and benefit information in an (online) environment, and in the predictors of online risk information sharing.

The Internet is one of the main sources currently used by individuals to search for information about food (Jacob, Mathiasen, \& Powell, 2010; Kuttschreuter et al., 2014; Redmond \& Griffith, 2006; Tian \& Robinson, 2008). When browsing the Internet, individuals are increasingly likely to end up on social media sites where they encounter the opinions of others. Compared to traditional media and face-to-face communication, online interaction has different characteristics (Dellarocas, 2003). For example, social media offers new possibilities for information transfer (Rutsaert et al., 2013a; Veil, Buehner, \& Palenchar, 2011). Social media, for instance, empower individuals to interact with others and express their own opinion (Shao, 2009), resulting in an increase of available information, public involvement and interaction (Rutsaert et al., 2013b). Also, on social media, such as blog-sites, users are essentially anonymous or can pretend to be someone other than who they really are (Dellarocas, 2003; Rutsaert et al., 2013a). One consequence may be that individuals act differently when they are anonymous in an online setting compared to being not anonymous in a face to face setting. For example, they may express themselves more freely and feel less restricted to behave in line with social norms (Bordia, 1997). Research also indicates that in online social 
interactions individual differences (in position or status) are less pronounced and thus shape the interaction less, leading to a more equal distribution of information sharing in groups that communicate in an online anonymous environment compared to face to face situations (Straus, 1996).

An important feature of the research presented in this dissertation is its emphasis on the exchange of risk information online with others. Previous risk research mainly focused on intra-personal processes connected to the searching for risk information, whereas the function of inter-personal sharing and exchange of online risk information and its determinants has not been given much attention yet. Based on the new opportunities that social media offer to (risk) communication, we make a distinction in three types of online information exchange.

First, we investigate social influence on social networking sites, focusing on Facebook. On these sites individuals can read and respond to comments and information posted by peers and other individuals or organisations in their network. Secondly, we focus on online interaction via a chat. Virtual chats are one of the new possibilities of the Internet and social media. This type of information exchange calls for a higher level of involvement compared to merely reading (and possibly being influenced by) information on social networking sites. Third, we focus on online information sharing and its determinants. This type of information exchange involves individuals actively sharing information they encounter with others via online media, for example by means of Twitter, Facebook, email or online chats.

Generally, examining information exchange is important because individuals are influenced by their social environment, which is convincingly and consistently demonstrated by research in other domains such as e.g. the effects of electronic word of mouth on product sales (e.g. Davis \& Khazanchi, 2008), and the success of new product introductions (e.g. Clemons, Gao \& Hitt, 2006). If it is known how individuals are influenced by social media information and what motivates individuals to share information themselves, information sharing behaviour can be enhanced, which arguably benefits to informed decision-making regarding food choices. To get insight in these processes, online information exchange about organic food risks is a central aspect in my dissertation.

\section{Organic food as topic}

The research is outlined along the topic of technological developments in food processing. Nowadays, one regularly observes (re)new(ed) food products in grocery stores. They often have a clear logo or label on the packaging, for example "organic", 
"natural" or "healthy choice". Some of these products are presented as healthier for individuals, for example, potato chips prepared with sunflower oil. Others emphasize that the products are produced in a way that is more efficient to the producer, better for animal welfare or the environment, such as free-range chickens. When individuals are unfamiliar with these products, they usually have not formed solid opinions about them yet and are inclined to use heuristics (i.e. mental shortcuts) to process information and evaluate the risks (Tversky \& Kahnemann, 1974). How individuals perceive risks of new food production techniques has an effect on the acceptance of the production method (Köhler \& Som, 2008; Sjöberg, 2004). Individuals might become unwilling to buy the products, because they may perceive new food producing methods as more dangerous compared to traditional ones (Siegrist, 2000). Therefore, it is important to examine individual's reactions to these products to be able to adequately anticipate on the information the individuals needs to make well-informed choices.

Especially in the food domain, there is a preference for naturalness and foods produced without human intervention (Rozin et al., 2004). Sjöberg $(2000,2004)$ argued that new technologies, such as nuclear technology and gene technology, are often perceived as unnatural and immoral. To what extent a technology is associated with 'tampering/inference with nature' appears to be an important predictor of risk perception, even more important than the factors that constitute perceived dread (e.g. perceived lack of control, catastrophic potential, fatal consequences) of the risk (Sjöberg, 2000; Sjöberg, 2004).

How individuals perceive the chance of a risk to occur and its consequences, e.g. their risk perception, is a complex process. Based on Slovic's research and his psychometric paradigm (e.g. Slovic, 2000, Slovic, Finucane, Peters, \& MacGregor, 2004), it is assumed that risk perception includes both cognition as well as affect. Especially anxiety is an important emotion related to risk perception. Risk-as-analysis means that a cognitive, logical, effortful and deliberate process results in risk perception, while risk-as-feelings states that how we feel about a certain risk directly influences our risk perception.

Though new technologies are often perceived as dangerous (e.g. Siegrist, 2000), organic food is generally perceived as positive (e.g. Magnusson et al., 2001; Saba \& Messina, 2003). This contradiction makes organic food an interesting research topic. On the one hand one would expect individuals to be hesitant towards organic food as this is a new technology used for food production, on the other hand organic food production is a technique that scores low on the tampering with nature dimension, and therefore is likely to be perceived as positive.

Organic products need to meet criteria regarding production methods, labeling and check-ups. These criteria are described by the European Commission in a regulation document (EC Regulation No. 834/2007). Organic foods are grown without synthetic 
pesticides, without synthetic fertilizers, and with extra attention for the environment, biodiversity, and animals (Ahmad, 2010). Animals can be breed in an organically responsible way. For example, these animals can roam freely. The downside of this is that they have a higher risk to get infected. In addition, on average, organic eggs have a larger negative effect on the climate compared to non-organic eggs (Tidwell, 2009). While organically produced foods are becoming more and more common and people generally have a positive attitude towards them, focussing on organic food also allows us to get insight in individuals' reactions and information behaviour related to a new risk that is likely to be underestimated.

The main research question of this dissertation is: How do individuals make sense of (online) risk information about (organic) food issues? The answer to this question is of particular importance for the Netherlands Food and Consumer Product Safety Authority. One of the tasks of the Netherlands Food and Consumer Product Safety Authority and equivalents in other countries, is to ensure that the products that are sold in grocery stores, kitchens in restaurants or institutions, and specialty stores are safe to eat. They also inform individuals about the possible risks of food products. To accomplish this goal and to adequately anticipate on individuals' needs, it is valuable for the authorities to know how individuals deal with food risk information. Information about how individuals make sense of food risk information, creates the opportunity to food communicators to adjust information messages in a way that individuals are better able to understand them, more easily make sense of the provided information, and are consequently better able to make well-informed decisions. It is yet unclear how individuals' sense-making process is influenced by their social environment in an online context.

\section{The sense-making theory}

To understand how individuals make sense of risk information I will take a usercentred approach. The development of the Internet and social media has created the possibility for individuals to seek, find, and share information themselves rapidly. It is thus important to look at the active role of individuals in the communication process of food information.

A user-centred approach is also the main focus of the sense-making theory (Dervin, 1983; Klein et al., 2006a, 2006b; Russell, Stefik, Pirolli, \& Card, 1993; Weick, 1995). Sense-making is the process by which individuals give meaning to the world around them, and sense is the outcome of this process. Sense-making involves recognizing a problem, seeking, finding and integrating new information in a way that there is no 
tension between the newly encountered information and one's own vision and beliefs (Weick, 1995; Weick, Sutcliffe \& Obstfeld, 2005). Individuals construct their reality by using their own observations and the observations of others (Dervin, 1983). The sense-making theory was introduced by Brenda Dervin $(1983,1992,1998,1999)$ to information science. Subsequently, it has been approached as macro-cognition theory by Klein et al. (2006a, 2006b), used in intelligence analysis by Pirolli and Card (2005), and applied in organisational studies by Weick (1995). The abundance of research on sense-making can be subdivided in three substantially different perspectives on sensemaking, which are discussed below (see also Pirolli \& Russell, 2011).

The first perspective is the representation construction model of sense-making. According to Russell, Stefik, Pirolli, and Card (1993) 'Sense-making is the process of searching for a representation and encoding data in that representation to answer task-specific questions' (p.269). Their model proposes that individuals sequentially develop more refined representations and use these representations to organize newly encountered information. The sense-making process consists of two loops of activities: the foraging loop (Pirolli and Card, 1999), in which information is encountered, searched and filtered, and the sense-making loop (Russell et al., 1993), in which this information is put in schema, hypotheses are derived, evidence is collected and a representation is formed (Qu and Furnas, 2005).

The representation construction model has been used in a variety of studies. For example, research examined self-directed learning (Butcher \& Sumner, 2011), the use of external representations in conducting large legal investigations (Attfield \& Blandford, 2011), and the use of external representations to make sense when searching online (Abraham, Petre \& Sharp, 2008).

The second perspective views sense-making as a cognitive activity. In this perspective sense-making is an individual cognitive activity that has meaning and knowledge as an outcome. Within the second perspective there are two major approaches. The first approach was developed by Brenda Dervin and departs from the notion that gapbridging is a useful metaphor to understand how individuals seek, interpret and design information (Savolainen, 2006). It consist of three elements: the situation, the gap, and uses/help. The context in which the sense is created is called situation. The gap refers to the information an individual needs or the questions ahead that need to be answered to achieve 'sense'. The gap is a state in which sense or meaning is lacking or incomplete, and is defined by discontinuity. The uses/helps refer to the cognitive bridges that are formed, e.g. the cognitive activities that involve how the individual interprets the information and the problem-solving activity.

The second approach extended the situation-gap-uses/help approach and transformed this model into a macro cognitive theory of sense-making (Klein et al., 
2006a, 2006b). This approach is characterised by the data/frame model. Klein et al. (2006a, 2006b) argue that individuals start with a minimal framework when they try to make sense of all kinds of data. Frames define what data is relevant and shape how we interpret the data. Sense-making involves connecting with a frame, elaborating a frame, questioning a frame, and reframing if necessary.

The second perspective on sense-making is mainly used in communication, library and information technology sciences, for example to understand what gaps individuals encounter when searching online for information (Savolainen, 2006), to test how women make sense of health information related to the menopause transition (Genuis, 2012), and to understand decision making of submarine commanding officers (Dominguez, Long, Miller, \& Wiggins, 2006).

The third perspective, collaborative sense-making, is often studied in organisational studies examining the ways in which groups of individuals create and structure information together. Karl Weick (1995) introduced the concept of sense-making on an organisational level, stressing the importance of social interaction and arguing that sense-making is closely related to the general process of organizing (Weick et al, 2005). He focuses on how individuals in teams structure chaos, unknown, uncertain and ambiguous events and rationalize what they are doing.

Researchers used the collaborative perspective for various goals, such as explaining leadership and the organizing of music teams (Humphreys, Ucbasaran \& Lockett, 2012), experiences of business leaders (Maclean, Harvey \& Chia, 2012), and the effect of storytelling within companies on sense-making (Näslund \& Pemer, 2012). This perspective was also used to get insight in collaborative sense-making of rally races to prevent incidents (Wahlström, Salovaara, Salo \& Oulasvirta, 2011), sense-making in collaborative web search (Paul \& Morris, 2011), and investigating the stories surrounding the financial crisis and how they shaped how the crisis was made sense of and acted upon (Whittle \& Mueller, 2012).

The first and second perspective focus on the individual but differ in the sense that the first perspective focuses on representation creating and using, while the second perspective focuses more on meaning and knowledge as outcome of the sense-making process. The third perspective focuses on group dynamics, and stresses how individuals structure unknown events to be able to act.

Although the three perspectives are somewhat different, the core is the same: Individuals encounter something (information, gap, data, unknown event) and try to create meaning and understanding. This process involves cognition (thoughts) and affect (emotions), as well as behaviour (actions) (Dervin, 1998; Weick et al., 2005). Fundamental elements of the sense-making process are information seeking, finding, processing, using, creating, and sharing (Dervin, 1992; Pirolli and Russell, 2011; 
Savolainen, 1993). Insights from these sense-making perspectives are useful to examine how individuals deal with food risk information regarding organic produce. Taken the insights from the three sense-making perspectives together, I view sense-making as the process of integrating and interpreting information to create a coherent entirety.

\section{This dissertation}

In relation to sense-making of food risk information, I argue that the following subtopics are important: initial attitudes and associations, and social influences in relation to information exchange. The sense-making process starts with information encountering. However, how individuals respond to this new information is predicted to be influenced by one's initial attitudes and viewpoints (Van Dijk, Fischer, De Jonge, Rowe, \& Frewer, 2012). A very first start to studying sense-making is thus making the initial attitudes, ideas, and associations of individuals regarding organic food explicit.

After encountering information, individuals process the information. Information processing is an individual cognitive process. These processes are likely to be influenced by the social environment and social media interaction. When individuals have processed the information, they can decide to share (one-way) or exchange (twoway interaction) this information with others. In a similar vein, other individuals in one's social environment can share their knowledge as well. This information exchange can elicit information seeking behaviour, and the process may restart.

In this dissertation, I particularly focus on the effects of the online social environment on information behaviour. In six empirical studies reported in four empirical chapters (chapter 2-5), I examine word associations with organic food, how individuals are influenced in their risk perception and sense-making by their online social environment (networking sites and online chat), and what motivates them to share information online with others. Please see Table 1.1 for an overview of the research questions, methodological approach, and outcome variables per chapter.

The first two empirical studies (reported in chapter 2) are conducted to provide insight in the initial attitude that individuals have towards organic food. We examine what word associations individuals have with organic food and which are most central, how associations differ between food type and consumer group, and what characteristics (psychological distance, values and socio-demographics) differentiate consumer groups (based on purchasing behaviour). This research extends the scope of current consumer research about organic food and provides new insight in the word associations consumers have with eating organic. 
How individuals make sense of food risk information is likely influenced by their initial attitude. By examining the words that come to individuals' mind when they think of organic food, insight is gained into how organic food is perceived. Construal Level Theory (Trope \& Liberman, 2010) is used as a theoretical framework to interpret the associations. This theory states that objects can vary from psychological close to distant. Psychological distant concepts can be defined as entities that are not present in the direct experience of reality, and can be distant on temporal (time), spatial (space/ location), social (relatedness to someone else/another group), and hypothetical (likelihood of an imaginary entity becoming reality) dimensions. Using Construal Level Theory is one of the novel aspects of our investigation.

Previous studies on organic food mainly focused on motives and barriers to buying organic food. How word associations differ between broad categories (food) and more narrow categories (meat and vegetables) and how psychological distance differs across consumer groups who differ in the frequency of buying organic foods is still unclear. In the first study of chapter 2 ( $n=154)$, we use a free response format to examine what word associations come to mind if consumers think of organic food, organic meat, or organic vegetables. In addition, consumer groups are compared on psychological distance, values, age and gender. In the second study reported in chapter 2 ( $n=52$ ), consumers' word associations from the first study were rated on centrality by an independent participant sample. Centrality can be defined as how characteristic consumers perceive the word associations to be to the concept of organic food/meat/ vegetables. Both studies make use of convenience samples.

In the third and fourth empirical study (reported in chapter 3 ) we focus on the influence of the social environment on sense-making. As social media offer new possibilities regarding information communication, it is decided to firstly focus on responses to information on social networking sites. To get an idea of how individuals are influenced by risk and benefit information that they encounter on social networking sites, two studies are performed. Facebook is used as social networking site in these two studies. Facebook gained popularity in the Netherlands the last years and is currently the most popular social media channel among university students (Cheung, Chiu \& Lee, 2011). Individuals use Facebook for various reasons, including interpersonal communication, maintaining relationships (Cheung et al., 2011), and information seeking (Basilisco \& Cha, 2015) about, for example, consumer trends (Asghar, 2015). The popularity and possibilities of Facebook make it relevant to examine to what extent individuals are influenced by proof of the opinions of others, in particular online comments and the number of likes at a statement. 


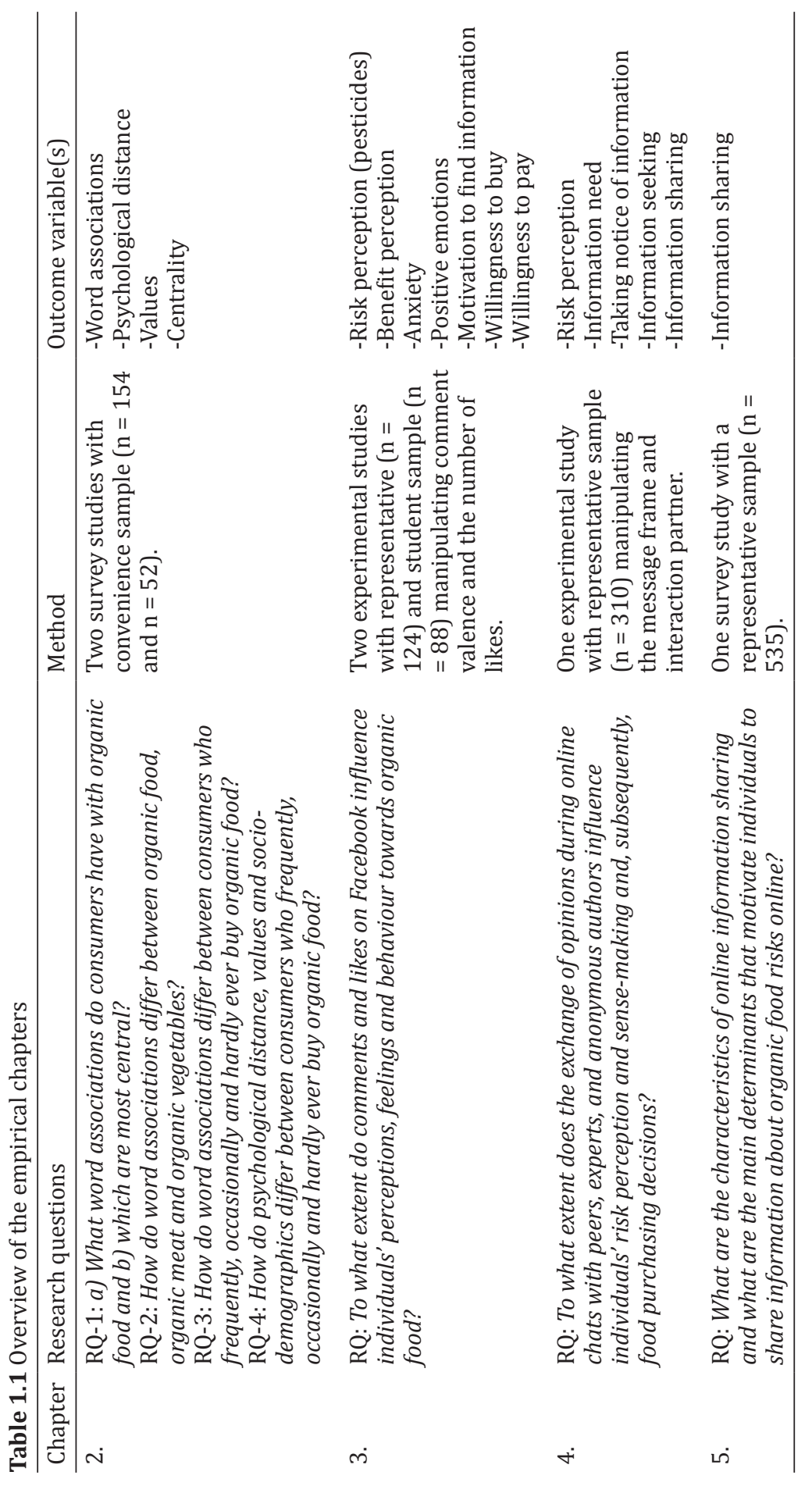


Individuals can post a statement or message on Facebook (a post), respond to these posts (the comments) and indicate that they agree with the post and/or comment by using the "thumb up" symbol (the likes). Comments on Facebook are often in favour or against a certain topic. This positivity or negativity is called 'valence'. My co-authors and I investigate the effect of Facebook comments and likes on perceptions, feelings, and behavioural intentions towards eating organic food in two online experimental studies. Specifically, we focus on how reactions on Facebook influence the way individuals make sense of food risk information, looking at both the comment valence and number of likes on Facebook pages. In previous research, comment valence has been found to have an effect on risk perception, while the results of the number of likes were mixed in previous studies.

The Facebook chapter consists of two experiments. In the first experimental study comment valence (positive versus negative) and the number of likes (high versus low) are manipulated. The evaluation of the comments in terms of clearness and usefulness is included as a moderator based on research of Slater and Rouner (1996). They argued that the evaluation of a message has an effect on source credibility and in turn influences message acceptance and belief change. In this study a representative sample of Dutch internet users $(n=124)$ with respect to gender and age is used.

In the second study, a full Facebook page with positive and negative statements is shown, and either the positive or the negative statements are reinforced by likes. This Facebook page is expected to be a closer resemblance of reality. A student sample $(n=88)$ is used to examine the effect of using likes as reinforcement of statements. Combining these two studies provides insight in the effects of comment valence, the number likes, and the combination of comments and likes.

In the fifth study (reported in chapter 4) my co-authors and I want to go one step beyond social networking sites and aim to tap into online sense-making and risk perception by letting participants evaluate the risks associated with organic food products in collaboration with virtual others (peer versus experts versus anonymous author). In this study, online information exchange is operationalized as an online chat simulation. We examine whether 1) the message frame, 2) the conversation partner; his/her perceived similarity and expertise, and 3) the individual's pre-experimental attitudes affect how individuals deal with food risk and benefit information in an online communication setting.

Nowadays, the Internet is one of the main sources to search for information about food (Jacob, Mathiasen, \& Powell, 2010; Kuttschreuter et al., 2014; Redmond \& Griffith, 2006; Tian \& Robinson, 2008) and individuals may end up on social media sites when searching. Social media contain the opinions of others, for example peers, experts or anonymous authors. Both peers and experts have been found to be influential on 
individuals' attitudes and behaviour in an offline context (e.g. Griskevicius, Cialdini, \& Goldstein, 2008; Pornpitakp, 2004; Andsager, Bemker, Choi, \& Torwel, 2006), while the influence of anonymous others is rather understudied. The current importance of social media as an online information source raises the question: to what extent does the exchange of opinions during online chats with peers, experts and anonymous authors influence individuals' risk perception and sense-making and, subsequently, food purchasing decisions? The opinion of the conversation partner can be presented in different ways. This is called message framing (Chong \& Druckman, 2007). We use three types of frame in our study: gains frame (e.g. emphasis on advantages), losses frame (e.g. emphasis on disadvantages), and uncertainty frame (no clear emphasis). This is in line with the distinction between promotion-focus and prevention-focus made in Higgens' Regulatory Focus Theory (Higgens, 1997).

An online interaction experiment, including a simulated chat in which we manipulate the conversation partner (expert vs peer vs anonymous interaction partner) and the message frame (gains vs losses vs uncertainty) is conducted to get insight in this issue using a representative sample of Dutch internet users $(n=310)$.

In the sixth study (reported in chapter 5), the third new opportunity of social media, information sharing, is examined more closely. In my opinion, information sharing is an essential aspect of the sense-making process as it is both a means to sensemaking as well as an outcome of the process (Yang, Kahlor, \& Griffin, 2013). Sharing (one-way communication) or exchanging information (two-way interaction) can be seen as a way in which the interaction among individuals enhances sense-making (Caughron et al., 2013) and the social construction of meaning (Miranda \& Saunders, 2003). Information seeking and processing are extensively studied (Yang, Aloe \& Feeley, 2014), but (online) information sharing has been given little attention. Therefore we examine what determinants are important in predicting online information sharing with others and to what extent information sharing behaviour is influenced by close others. Most research on information sharing has been conducted in relation to the sharing of information in teams or workgroups in order to study its impact on group performance. Little attention has been given to what motivates individuals to share information online in a broader societal context (Yang, Kahlor, \& Griffin, 2013). The available research on the motives to share information via social media mostly focused on the characteristics and gratifications of the media channel (e.g. Oh \& Syn, 2015), and it did not specify the topic and content of the information that was to be shared. This study adds to the existing literature by examining online information sharing behaviour about organic products and their risks. We use variables from the RISP-model, the Theory of Planned Behaviour and constructs from previous studies on information seeking and/or sharing to explain information sharing, giving special attention to the role of the interest within 
the social environment and social norms to see if sharing behaviour is influenced by close others. An online survey among a representative sample of 535 respondents is conducted to examine the determinants information sharing behaviour, and their relationships. Structural equation modelling is applied to test both the measurement model and the structural model. 
The sales of organic food products are increasing and eating organic is becoming more popular (Hughner et al., 2007). Research about organic food is extensive, mainly focusing on the reasons for buying organically produced food. These reasons are called "motives". This research showed that the concern for health and wellbeing is central to consumers' purchase motives (Hughner et al., 2007), that consumers differ in their level of experience and knowledge regarding organic food, that consumers with different levels of experience perceive organic food differently (Zanoli \& Naspetti, 2002), and that these perceptions have an effect on attitudes towards eating organic, which in turn influence the intention to purchase organic food (Lee \& Yun, 2015). Though previous research provides insight into the motives to buy organic food and how individuals perceive organic food, consumers' word associations with organic food and how central these word associations are perceived to be haven't been given much attention.

Developing a coherent framework of consumers' word associations with the concept of organic food is important as this gives insight into how consumers make sense of this type of products. This insight would enable authorities to adapt their information supply to empower consumers to make well-informed choices about purchasing organic food. It might also help to understand why certain products are preferred over others.

This article presents the results of two studies. In study 2.1 it was examined what word associations come to mind if consumers think of organic food, organic meat, or organic vegetables. It was also investigated how psychological distant (or close) consumers experience organic food. Psychological distant concepts can be defined as entities that are not present in the direct experience of reality, and can be distant on temporal (time), spatial (space/location), social (relatedness to someone else/ another group), and hypothetical (likelihood of an imaginary entity becoming reality) dimensions (Trope \& Liberman, 2010).

A distinction between consumers who frequently, occasionally and hardly ever buy organic food was made to examine how these groups differ regarding their word associations, psychological distance, their values, gender, and age. In study 2.2, consumers' word associations from the first study were rated on centrality by an independent participant sample. Centrality can be defined as how characteristic consumers perceive the word associations to be to the concept of organic food/meat/ vegetables.

To our best knowledge, our psychological approach and methodology has not yet been used to analyse how people think of organic food, organic meat and organic vegetables. Our procedure of writing down word association in a first study and then have an independent sample rate these associations on centrality in a second study is an innovative approach in this research field. 


\section{Organic food}

Organic products need to meet criteria regarding production methods, labelling and check-ups. These criteria are described by the European Commission in a regulation document (EC Regulation No. 834/2007). Organic foods are grown without synthetic pesticides, without synthetic fertilizers, and with extra attention for the environment, biodiversity, and animals (Ahmad, 2010).

According to Hughner et al. (2007) consumers interpret the term "organic" in a variety of ways and in a multitude of differing contexts. To understand how consumers perceive organic food it is important to examine what word associations they have when they think of the concept and what associations are perceived to be most central.

\section{Word associations}

Consumers' word associations may depend on the broadness (i.e. diversity of products) of a food category. Organic food, organic meat, and organic vegetables differ in levels of broadness and thus word associations may differ with respect to the inclusion of specific features. This idea is reflected in the Construal Level Theory (Trope \& Liberman, 2010) that states that the more psychologically distant an object is, the more abstract the individuals' thoughts are, while objects that are close are related to concrete thoughts. We assume that broad categories, such as "food" are perceived as more distant than narrow categories, such as meat or vegetables, leading to possibly different word associations.

Psychological distance was subdivided in four dimensions by Trope and Liberman (2010). First, they distinguish temporal distance, which indicates whether an object is distant or close in terms of time. Objects in the present are low in temporal distance, while objects in the future are temporal distant. Other dimensions of psychological distance include spatial distance (distance in space/location), social distance (relatedness to someone else/another group), and hypothetical distance (likelihood of an imaginary entity becoming reality).

Fiedler (2007) adds informational and affective distance. Informational distance is expressed as the amount of information a consumer has about decision options, with the more dense the information the lesser the distance. If consumers feel uninformed about organic food, thinking of organic food might result in other word associations compared to consumers who are feel well-informed. Affective distance is the 'warm' or 'cold' feeling towards organic food (Fiedler, 2007). Emotional closeness, for example shown in perceiving eating organic as a way of life (Schifferstein \& Ophuis, 1998), might differ across consumer groups and elicit different word associations. 
Besides predicted differences in psychological distance, different aspects of the organic production may be relevant for different product types. Animal welfare is related to meat production, while cultivating crops without pesticides is relevant for organic vegetables. Because these two types of food products tap into different aspects of organic food production, the word associations connected to the food types are predicted to vary.

\section{Motives to buy organic}

Hughner et al. (2007) performed a review study investigating why consumers buy organic food. This review extracted nine motives for buying and six motives for not buying, that were supported in more recent literature (e.g. Lee \& Yun, 2015). Consumers were mostly motivated to buy organic for reasons related to health (Harper \& Makatouni, 2002; Roitner-Schobesberger et al., 2008; Padel \& Foster, 2005; Zanoli \& Naspetti, 2002), including wellbeing (Zanoli \& Naspetti, 2002) and nutritional values (Lea \& Worsley, 2005). Secondly, taste was found to be an important motive to buy organic (Lea \& Worsley, 2005; Roitner-Schobesberger et al., 2008; Schifferstein \& Ophuis, 1998). Other important motives were environmental concern (Lea \& Worsley, 2005; Magnusson et al., 2001; Roitner-Schobesberger et al., 2008) and animal welfare (Harper \& Makatouni, 2002).

Motives that were more peripheral for consumers to purchase organic food included concern over food safety, supporting local economy, being wholesome, nostalgia and fashionableness (Hughner et al., 2007).

Besides motives to buy organic food, consumers experience several motives to not buy organic. Most important motives that prevent consumers from buying organic are price and lack of availability of organic food (Lea \& Worsley, 2005; Magnusson et al., 2001; Zanoli \& Naspetti, 2002). Other motives include being sceptic towards organic food authenticity, being satisfied with conventional food, and perceiving organic food to be less attractive compared to conventional food (Hughner et al., 2007). Researchers also inferred that insufficient marketing of organic products was a motive not to buy (Hughner et al., 2007).

While motives and barriers to buy organic food provide some insight in how consumers make sense of organic food, they only give limited information about the word associations that come to consumers' minds. 


\section{Differences between consumers}

The concept of organic food can be viewed as a relational concept (Eden, 2009). This means that, besides common sense-making (Miranda \& Saunders, 2003), the meaning of the concept is created by the consumers' perspectives, implying that different consumer groups might differ in word associations. Consumers may vary in levels of experience and knowledge of organic food, for example observable in purchasing habits and psychological distance.

Consumers may also differ with respect to values and sociodemographic-background.

\section{Consumers' values}

Values are beliefs about what is important in our lives, and motivate action (cf. Schwartz, 2012). Three main categories of life values were defined by Makatouni (2002): human, animal, and environment centred values. These values were found to be predictive of organic food purchase behaviour. Lea and Worsley (2005) found that the universal personal value factor, which included human and animal centred values, was the most important predictor of positive beliefs regarding organic food. Gender was found to be the second dominant predictor.

Values were also found to differ across consumer groups. Harper and Makatouni (2002) differentiated three types of consumers: non-organic produce consumers, occasional organic produce consumers, and regular organic produce consumers. Regular consumers were more concerned about their health and were more interested in ethical issues compared to the other two groups. Non-organic produce consumers were not concerned about food safety, while the other groups were, and their food choice was mainly driven by financial reasons. Other research showed that frequent and occasional buyers perceived organic food differently (Zanoli \& Naspetti, 2002). While both frequent and occasional buyers valued health, the occasional consumers lacked values like altruism and the realisation of a sustainable future in comparison with frequent buyers, who had a more idealistic value pattern. The occasional consumers were particularly attracted by taste and pleasure resulting from eating organic produce.

\section{Research questions}

This research aimed to answer the following four research questions:

RQ-1: a) What word associations do consumers have with organic food and b) which are most central?

RQ-2: How do word associations differ between organic food, organic meat and organic vegetables? 
RQ-3: How do word associations differ between consumers who frequently, occasionally and hardly ever buy organic food?

RQ-4: How do psychological distance, values and socio-demographics differ between consumers who frequently, occasionally and hardly ever buy organic food?

\section{Method study 2.1: Word associations, psychological distance and values}

\section{Participants and Design}

This study was performed in accordance with the ethical standards of the institutional research committee. A convenience sample was recruited via the social network of the researchers, and blogs and fora that discuss food in general and/or organic food in particular. Participants were requested to fill out an online questionnaire in Dutch, which took about 20 minutes. Data collection took place in June/July 2015.

Participants were divided into three consumer groups based on their reported purchase behaviour regarding organic food: consumers who frequently $(n=46)$, occasionally $(n=60)$, or hardly ever bought organic food $(n=48)$. We aimed at an equal distribution of participants across these groups. Therefore participants were excluded from participation when their consumer group already consisted of 60 participants. The sample consisted of 154 participants who were aged between 15 and 77 years old $(M=36)$. The sample consisted of 41 males $(27 \%)$ and 113 females $(73 \%)$. The participants mainly had a higher education (76\%). A total of $19 \%$ had a professional education and $6 \%$ a secondary education. A total of $52 \%$ of the participants could live (very) comfortably with their financial situation, $38 \%$ could get by, $9 \%$ had difficulty or struggled to get by, and $1 \%$ responded with "I don't know".

To avoid a bias in listing the word associations between the broader concept of organic food and specific product types, two conditions were used. Half of the participants were asked to generate word associations with organic food $(n=80)$ and the other half with organic meat and organic vegetables $(n=74)$. In the latter case the two product types were counterbalanced. A randomisation check was performed to ensure that observable participant characteristics were balanced across the studied food types (Bruhn \& McKenzie, 2009). This check showed that there were no differences between the three groups with respect to purchase behaviour, gender, age, financial situation and education. 


\section{Measurement}

All measures, scale characteristics and analysis procedure per construct are shown in Appendix 2.A. Cronbach's alpha was computed for all constructs as a lower bound estimate of reliability. A reliability of .60 is considered to be acceptable (Loewenthal, 2004).

Word associations. Participants were requested to "list as many single word associations or sentences up to 6 words as came to mind, and to include the obvious, though not to take more than five minutes for this task". This question was based on Fehr (1988).

Psychological distance. Psychological distance was subdivided in temporal distance $(\alpha=.76)$, social distance $(\alpha=.82)$, spatial distance $(\alpha=.81)$, hypothetical distance $(\alpha=$ $.91)$, informational distance $(\alpha=.90)$, and affective distance $(\alpha=.92)$. Items were newly created for the purpose of this study using insight from Trope and Liberman (2010) and Fiedler (2007) as a starting point. An explanatory factor analysis confirmed the expected structure, albeit that hypothetical and affective distance loaded on the same factor.

Values. Values regarding the environment ( $\alpha=.87)$, human health $(r=.59)$, animal welfare $(\alpha=.86)$, price/quality $(\alpha=.85)$, and attractiveness of food products $(\alpha=.58)$ were measured. Items were newly created for the purpose of this study using insight from Makatouni (2002) as a starting point. The price and attractiveness items loaded on separate factors; environmental, human health and animal items loaded on the same factor. As environmental, human and animal values differed substantively content wise, these subscales were kept separate in the analyses.

\section{Procedure}

Study 2.1 was an online survey. Before starting the online survey, participants gave informed consent to participation and were informed about the purpose of this study. Word associations with 'the Netherlands' were provided as an example. After the listing of word associations, psychological distance, values, and socio-demographic variables were measured.

\section{Data-analysis}

The procedure for coding the word associations was adapted from Fehr (1988). A total of 1088 associations were obtained. Responses that concerned comments or were unrelated to the topic were deleted, resulting in 1066 usable responses. All responses were categorised by two judges; a graduate and a postgraduate student in psychology. Categorisation was discussed until consensus was reached. 
The coding of the word associations resulted in a total of 29 categories (Appendix 2.B). The coded associations were listed for each of the food types (food, meat, vegetables) and compared across consumer groups (frequently, occasionally and hardly ever organic buyers). Because both food types as well as consumer groups differed with respect to the number of participants, these frequencies were adjusted in a way that each group represented 100 participants. The frequencies were adjusted to avoid that the differences in number of word associations between groups (food types, and consumer groups) could be explained by the number of participants in each group. The unit of analysis is the list of associations per subcategory of food and consumer group rather than the individual participant.

The consumer groups were also compared on psychological distance, values and socio-demographics.

\section{Results Study 2.1}

\section{Word associations}

The absolute and adjusted frequencies of the word associations were listed per food type (Table 2.1) and consumer group (Table 2.2). With respect to RQ-1, overall, word associations concerning "animal welfare", "price", "health", "pesticide use", and "naturalness" were mentioned most often.

\section{Food types}

Of the categories that were mentioned most often, "animal welfare" was more often mentioned regarding meat than food or vegetables. Meat scored significantly lower on "naturalness" and "health" compared to both food and vegetables, while there was no difference between food and vegetables. Vegetables showed the highest frequency for "pesticide use". No differences in frequency of "price" were found.

With respect to the other word associations (nr. 6-29), respondents least often mentioned the "environment" and specific "products" regarding meat compared to food and vegetables, and significantly more often "medicine" and "sceptical remarks". Respondents most often mentioned the "origin", "appearance of food", "nutrition" and "safety" in relation to vegetables. "Additives" were mentioned most often when the cue was food or meat. "Shops and brands" were most often mentioned regarding food, but this was not significantly different from the frequency of vegetables. 
Table 2.1 The absolute and adjusted (between brackets; $n=100$ ) frequencies per food type

\begin{tabular}{|c|c|c|c|c|c|c|}
\hline \multirow[t]{2}{*}{ Category } & \multirow[t]{2}{*}{ Total } & \multicolumn{5}{|c|}{ Food type } \\
\hline & & $\begin{array}{c}\text { Food } \\
(\mathrm{n}=80)\end{array}$ & $\begin{array}{c}\text { Meat } \\
(n=74)\end{array}$ & $\begin{array}{l}\text { Vegetables } \\
(\mathrm{n}=74)\end{array}$ & $\chi^{2}$ & $p$ \\
\hline 1. Animal welfare & 138 & $31(39)^{a}$ & $104(141)^{b}$ & $3(4)^{c}$ & 165.21 & $<.001$ \\
\hline 2. Price & 106 & $41(51)$ & $27(36)$ & $38(51)$ & 3.26 & .20 \\
\hline 3. Health & 101 & $43(54)^{a}$ & $21(28)^{b}$ & $37(50)^{\mathrm{a}}$ & 8.91 & .01 \\
\hline 4. Pesticide use & 77 & $28(35)^{a}$ & $4(5)^{b}$ & $45(61)^{c}$ & 46.65 & $<.001$ \\
\hline 5. Naturalness & 71 & $35(44)^{a}$ & $10(14)^{b}$ & $26(35)^{a}$ & 15.29 & $<.001$ \\
\hline 6. Environment & 60 & $27(34)^{a}$ & $7(9)^{\mathrm{b}}$ & $26(35)^{a}$ & 16.69 & $<.001$ \\
\hline 7. Taste & 57 & $16(20)$ & $18(24)$ & $23(31)$ & 2.48 & .29 \\
\hline 8. Origin & 51 & $15(19)^{\mathrm{a}}$ & $8(11)^{a}$ & $28(38)^{b}$ & 16.97 & $<.001$ \\
\hline 9. Products & 42 & $22(28)^{a}$ & $6(8)^{b}$ & $14(19)^{\mathrm{a}}$ & 10.95 & .004 \\
\hline 10. Medicine & 39 & $8(10)^{\mathrm{a}}$ & $31(42)^{b}$ & $0(0)^{c}$ & 55.54 & $<.001$ \\
\hline 11. Scepticism & 31 & $2(3)^{a}$ & $21(28)^{b}$ & $8(11)^{c}$ & 23.29 & $<.001$ \\
\hline 12. Honesty & 30 & 15(19) & $6(8)$ & $9(12)$ & 4.77 & .09 \\
\hline 13. Unprocessed & 27 & 11(14) & $7(9)$ & $9(12)$ & 1.09 & .58 \\
\hline 14. Additives & 27 & $15(19)^{\mathrm{a}}$ & $10(14)^{\mathrm{a}}$ & $2(3)^{\mathrm{b}}$ & 11.17 & .004 \\
\hline 15. Quality & 22 & $5(6)$ & $9(12)$ & $8(11)$ & 2.14 & .34 \\
\hline 16. Sustainability & 20 & $5(6)$ & $8(11)$ & $7(9)$ & 1.46 & .48 \\
\hline 17. Safety & 19 & $5(6)^{a}$ & $3(4)^{a}$ & $11(15)^{b}$ & 8.24 & .02 \\
\hline 18. Appearance & 18 & $2(3)^{a}$ & $1(1)^{\mathrm{a}}$ & $15(20)^{\mathrm{b}}$ & 27.25 & $<.001$ \\
\hline 19. Nutrition & 18 & $5(6)^{a}$ & $2(3)^{a}$ & $11(15)^{b}$ & 9.75 & .01 \\
\hline 20. Availability & 18 & $7(9)$ & $3(4)$ & $8(11)$ & 3.25 & .20 \\
\hline 21. Lifestyle & 16 & $7(9)$ & $6(8)$ & $3(4)$ & 2.00 & .37 \\
\hline 22. Emotions & 14 & $6(8)$ & $2(3)$ & $6(8)$ & 2.63 & .27 \\
\hline 23. Shops/brands & 13 & $8(10)^{a}$ & $1(1)^{\mathrm{b}}$ & $4(5)^{a, b}$ & 7.63 & .02 \\
\hline 24. Quantity & 12 & $3(4)$ & $2(3)$ & $7(9)$ & 3.88 & .14 \\
\hline 25. Presentation & 10 & $2(3)$ & $5(7)$ & $3(4)$ & 1.86 & .40 \\
\hline 26. Trend & 9 & $6(8)$ & $0(0)$ & $3(4)$ & 1.33 & .25 \\
\hline 27. Certification & 8 & $2(3)$ & $5(7)$ & $1(1)$ & 5.09 & .08 \\
\hline 28. Check-up & 6 & $4(5)$ & $2(3)$ & $0(0)$ & 4.75 & .09 \\
\hline 29. Animals & 6 & $2(3)$ & $4(5)$ & $0(0)$ & 4.75 & .09 \\
\hline
\end{tabular}

Note: The frequencies were adjusted to equal sample sizes $(N=100)$ by dividing the original frequency by the original sample size per group times 100 .

Note: Adjusted frequencies were compared between food types using a $\chi^{2}$-test. When chi-squared showed a significant effect pairwise comparisons were made (non-parametric). Different superscripts $(\mathrm{a}-\mathrm{c})$ indicate a significant difference between the adjusted frequencies of the food types per category. 
Table 2.2 The absolute and adjusted (between brackets; $\mathrm{n}=100$ ) frequencies per consumer group

\begin{tabular}{|c|c|c|c|c|c|c|}
\hline \multirow[t]{2}{*}{ Category } & \multirow[t]{2}{*}{ Total } & \multicolumn{5}{|c|}{ Consumer group } \\
\hline & & $\begin{array}{l}\text { Frequently } \\
(\mathrm{n}=46)\end{array}$ & $\begin{array}{c}\text { Occasionally } \\
(\mathrm{n}=60)\end{array}$ & $\begin{array}{l}\text { Hardly ever } \\
(\mathrm{n}=48)\end{array}$ & $\chi^{2}$ & $p$ \\
\hline 1. Animal welfare & 138 & $49(107)$ & $50(83)$ & $39(81)$ & 4.64 & .10 \\
\hline 2. Price & 106 & $15(33)^{\mathrm{a}}$ & $40(67)^{b}$ & $51(106)^{c}$ & 38.86 & $<.001$ \\
\hline 3. Health & 101 & $35(76)^{a}$ & $43(72)^{\mathrm{a}}$ & $23(48)^{b}$ & 7.02 & .030 \\
\hline 4. Pesticide use & 77 & $25(54)$ & $33(55)$ & $19(40)$ & 2.83 & .24 \\
\hline 5. Naturalness & 71 & $36(78)^{a}$ & $23(38)^{b}$ & $12(25)^{b}$ & 32.47 & $<.001$ \\
\hline 6. Environment & 60 & $22(48)^{a}$ & $28(47)^{\mathrm{a}}$ & $10(21)^{b}$ & 12.12 & .002 \\
\hline 7. Taste & 57 & $27(59)^{a}$ & $25(42)^{\mathrm{a}}$ & $5(10)^{b}$ & 33.46 & $<.001$ \\
\hline 8. Origin & 51 & $20(43)^{a}$ & $13(22)^{b}$ & $18(38)^{\mathrm{a}}$ & 7.01 & .030 \\
\hline 9. Products & 42 & $6(13)^{a}$ & $21(35)^{b}$ & $15(31)^{b}$ & 10.43 & .005 \\
\hline 10. Medicine & 39 & $16(35)^{\mathrm{a}}$ & $16(27)^{\mathrm{a}, \mathrm{b}}$ & $7(15)^{\mathrm{b}}$ & 7.90 & .02 \\
\hline 11. Scepticism & 31 & $16(35)^{a}$ & $3(5)^{b}$ & $12(25)^{\mathrm{a}}$ & 21.54 & $<.001$ \\
\hline 12. Honesty & 30 & $12(26)^{a}$ & $14(23)^{\mathrm{a}}$ & $4(8)^{\mathrm{b}}$ & 9.79 & .007 \\
\hline 13. Unprocessed & 27 & $12(26)^{a}$ & $12(20)^{a}$ & $3(6)^{b}$ & 12.15 & .002 \\
\hline 14. Additives & 27 & $8(17)^{\mathrm{a}, \mathrm{b}}$ & $15(25)^{a}$ & $4(8)^{\mathrm{b}}$ & 8.68 & .013 \\
\hline 15. Quality & 22 & $12(26)^{a}$ & $9(15)^{\mathrm{a}}$ & $1(2)^{b}$ & 20.14 & $<.001$ \\
\hline 16. Sustainability & 20 & $9(20)$ & $7(12)$ & $4(8)$ & 5.60 & .06 \\
\hline 17. Safety & 19 & $9(20)^{\mathrm{a}}$ & $3(5)^{b}$ & $7(15)^{\mathrm{a}}$ & 8.75 & .013 \\
\hline 18. Appearance & 18 & $6(13)^{a}$ & $10(17)^{\mathrm{a}}$ & $2(4)^{b}$ & 7.82 & .020 \\
\hline 19. Nutrition & 18 & $10(22)^{\mathrm{a}}$ & $5(8)^{b}$ & $3(6)^{b}$ & 12.67 & .002 \\
\hline 20. Availability & 18 & $7(15)$ & $8(13)$ & $3(6)$ & 3.94 & .14 \\
\hline 21. Lifestyle & 16 & $7(15)^{\mathrm{a}}$ & $2(3)^{b}$ & $7(15)^{\mathrm{a}}$ & 8.73 & .013 \\
\hline 22. Emotions & 14 & $7(15)^{\mathrm{a}}$ & $6(10)^{a}$ & $1(2)^{\mathrm{b}}$ & 9.56 & .008 \\
\hline 23. Shops/brands & 13 & $5(11)^{a}$ & $7(12)^{\mathrm{a}}$ & $1(2)^{\mathrm{b}}$ & 7.28 & .026 \\
\hline 24. Quantity & 12 & $6(13)$ & $3(5)$ & $3(6)$ & 4.75 & .09 \\
\hline 25. Presentation & 10 & $2(4)$ & $5(8)$ & $3(6)$ & 1.33 & .51 \\
\hline 26. Trend & 9 & $1(2)$ & $3(5)$ & $5(8)$ & 5.77 & .06 \\
\hline 27. Certification & 8 & $4(9)$ & $2(3)$ & $2(4)$ & 8.88 & .14 \\
\hline 28. Check-up & 6 & $1(2)$ & $3(5)$ & $2(4)$ & 1.27 & .53 \\
\hline 29. Animals & 6 & $0(0)^{\mathrm{a}}$ & $2(3)^{a, b}$ & $4(8)^{b}$ & 8.91 & .01 \\
\hline
\end{tabular}

Note: The frequencies were adjusted to equal sample sizes $(N=100)$ by dividing the original frequency by the original sample size per food type times 100 .

Note: Adjusted frequencies were compared between consumer groups using a $\chi^{2}$-test. When chi-squared showed a significant effect pairwise comparisons were made (non-parametric). Different superscripts (a-c) indicate a significant difference between the adjusted frequencies of the consumer groups per category. 


\section{Consumer groups}

Regarding the most often mentioned categories, consumers who frequently buy organic food mentioned "naturalness" significantly more often than the other groups and "price" less often. "Health-related" associations were significantly less often mentioned by consumers who hardly ever buy organic food compared to both frequent and occasional consumers. The analysis showed no significant differences between consumer groups on "animal welfare" and "pesticide use".

Regarding the other word associations (nr. 6-29), consumers in the frequently group most often mentioned "nutrition", while they mentioned "examples of products" least often.

There was a significant difference in "medicine", which was more often mentioned by frequent consumers, and "examples of animals", which was more often mentioned by consumers who hardly ever buy organic, between the frequent and hardly ever consumer group. Consumers who hardly ever bought organic food mentioned word associations concerning "environment", "taste", "positive emotions", "quality", "appearance", "specific shops or brands", as well as organic food being "unprocessed" and "honesty" significantly less often than other groups.

The absence of "additives" was most often mentioned by the occasional group, though the difference with the frequent group was insignificant. Consumers in both the frequent and hardly ever consumer group mentioned word associations regarding "origin", "scepticism", "safety", and "lifestyle" more often than consumers in the occasional group.

\section{Consumers characteristics}

Socio-demographics. Consumer groups differed significantly with respect to gender, $\chi^{2}(2)=14.78, p=.001$, and age, $F(2,151)=3.84, p=.02$. Consumers who frequently purchased organic food were older $(M=39)$ compared to those who hardly ever purchased organic food $(M=32)$. There were no significant differences regarding education level and financial situation.

Psychological distance. There was a significant multivariate effect of the consumer groups on psychological distance, Wilk's $\lambda=.20, F(12,292)=30.62, p<.001, \eta^{2}=.56$, showing that consumer groups differed on their psychological distance to organic food products. This effect held for all the subscales.

Pairwise-comparisons, using a Bonferroni correction, showed that the distance to organic food for the frequently consumer was the smallest, and increased for the occasional and hardly ever consumer groups, respectively (Table 2.3). 


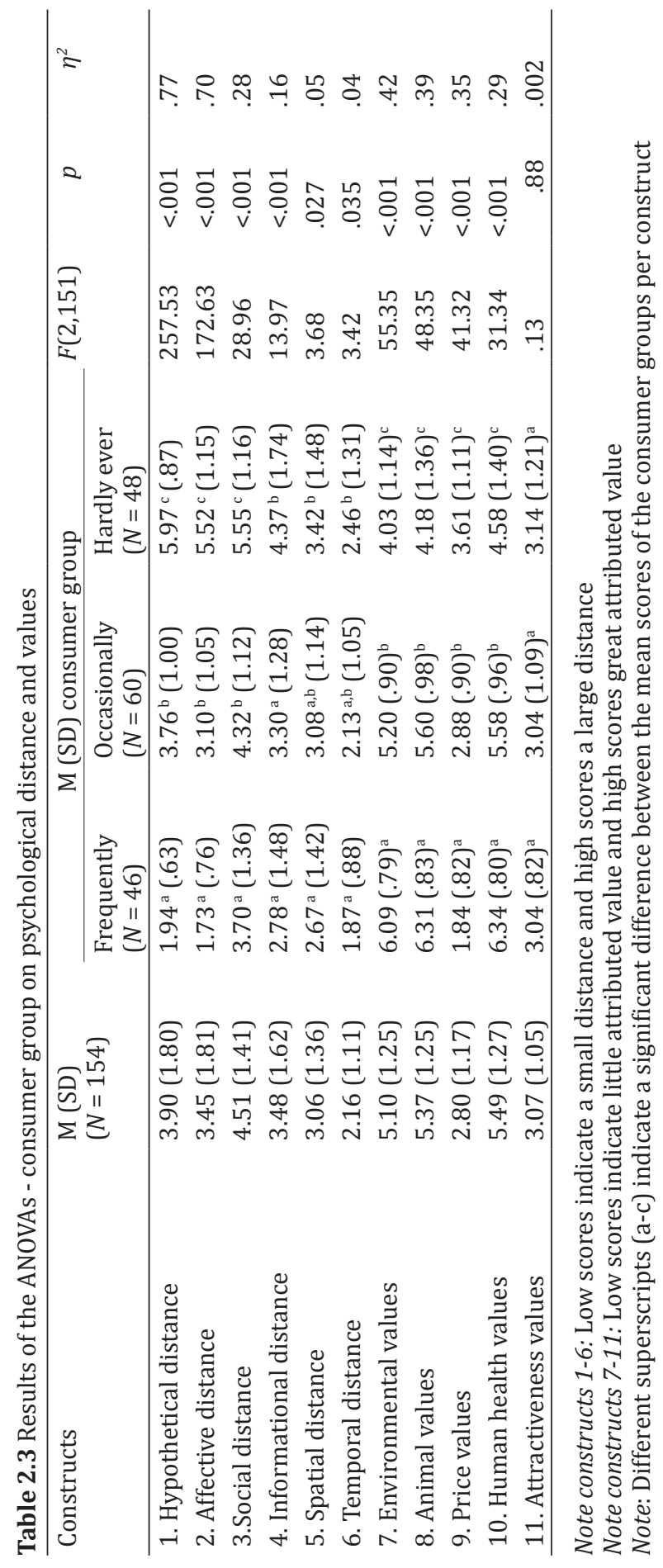


Hypothetical, affective and social distance differed between all groups. Regarding informational distance the hardly ever group scored significantly higher compared to both the frequent and occasional consumers, while there was no difference between frequent and occasional consumers. With respect to spatial and temporal distance, consumers who hardly ever buy organic food experienced significantly more distance compared to frequent buyers. No differences between the frequent and occasional consumer, and the occasional and hardly ever consumer were found.

Males scored significantly higher on hypothetical, $F(1,153)=20.82, p<.001$, informational, $F(1,153)=8.64, p=.004$, and affective distance, $F(1,153)=19.46, p$ $<.001$. No differences between males and females were found regarding social, spatial and temporal distance, all $p$ 's $>.05$.

Values. There was a significant multivariate effect of the consumer group on the values, Wilk's $\lambda=.45, F(10,294)=14.56, p<.001, \eta^{2}=.33$. This effect was a significant for all value subscales except for attractiveness-centred values. This pattern was identical for males and females. Bonferroni post-hoc analysis showed that the frequently group valued the environment, animals and their health the most and this decreased for the occasional and hardly ever groups, respectively. The reverse effect was found regarding price-values: frequent consumers attributed the least value to the price of organic food (Table 2.3).

\section{Method Study 2.2: Centrality ratings}

\section{Participants and Design}

This study was performed in accordance with the ethical standards of the institutional research committee. Participants were requested to fill out a paper-andpencil questionnaire, which took about 15 minutes. Data collection took place in August 2015.The convenience sample consisted of 52 participants who were aged between 1971 years old $(M=39)$. The sample consisted of 23 males $(44 \%), 26$ females $(50 \%)$, and 3 unknown (6\%). The respondents in study 2 mainly had a higher education (60\%), followed by professional education (31\%) and secondary education (4\%), and 3 unknown (6\%). A total of $46 \%$ participants could live (very) comfortably with their financial situation, $39 \%$ could get by, $6 \%$ had difficulty or struggled to get by, and $4 \%$ responded with "I don't know". A total of 19\% participants frequently bought organic food, $42 \%$ occasionally, and 33\% hardly ever.

Participants of Study 2.1 and Study 2.2 did not significantly differ on age, education and financial situation. They differed on gender, $\chi^{2}(1,154)=7.11, p=.008$, in a way that the distribution of males and females was more equal in study 2.2 , whereas females were overrepresented in study 2.1 . 
Two conditions were used: Analogously to study 2.1, in the first condition participants were asked to rate the associations with organic food $(n=26)$, in the second condition participants rated the specific product types (organic meat and organic vegetables; $\mathrm{n}=$ 26).

\section{Measurement and procedure}

In Study 2.2, an independent participant sample rated how central each category ${ }^{1}$ derived from study 2.1 was to organic food, organic meat or organic vegetables to discover which word associations were considered to be central and which to be more peripheral. Items were based on Fehr (1988). Before starting the questionnaire, participants gave informed consent to participation and were informed about the purpose of this study.

\section{Data analysis}

Fehr's (1988) median split method was used. This means that word associations with a higher centrality rating than the median were considered central; the remaining word associations were considered peripheral. The median of the centrality rating of 'food' was 5.08, of 'meat' 5.77, and of 'vegetables' 5.74.

\section{Results Study 2.2}

Word associations related to "environment", "health", "honesty", "pesticide use", "sustainability", “quality", "naturalness", “additives", “origin”, “certification”, and "taste” were central across all food groups (Table 2.4). In line with results from study 1, "animal welfare" was central to both food and meat, but not to vegetables. "Price" and "lifestyle" were uniquely central to food. "Check-up" and being "unprocessed" were central to meat and vegetables, but not to food. "Nutrition" was rated central only to vegetables. Word associations were generally rated lower on the centrality scale for food than meat or vegetables. These lower centrality ratings imply that the word associations overall were less central to the concept of organic food compared to organic meat or organic vegetables, and thus more psychologically distant. This is in line with the Construal Level Theory (Trope \& Liberman, 2010).

Comparing the centrality ratings with the frequencies from study 2.1 , centrality and frequency mainly correspond regarding food $(r=.67, p<.001)$ : the word associations that were mentioned most often in Study 2.1 had high centrality ratings. This relation was not significant for meat $(r=.20, p>.05)$ or vegetables $(r=.33, p>.05)$. This means

\footnotetext{
${ }^{1}$ The association 'medicine' was erroneously left out of this study.
} 
that word associations that consumers actively often thought of were on average not perceived as central, while word associations that were central were on average not mentioned often when participants wrote down their own thoughts. With respect to vegetables, "pesticide use", "origin", "environment" and "health" were mentioned most often, and their centrality ratings were high. "Check-up", "sustainability”, "honesty", and "additives" had the highest centrality rating, but were mentioned seldom. Regarding meat, word associations concerning the "environment", "sustainability", "check-up", and "honesty" had the highest centrality ratings, though they were mentioned only a few times in study 2.1 .

Table 2.4 Centrality ratings and adjusted frequencies ( $n=100$; Study 1$)$ of the associations

\begin{tabular}{|c|c|c|c|c|c|c|c|c|}
\hline \multicolumn{3}{|c|}{ Food } & \multicolumn{3}{|c|}{ Meat } & \multicolumn{3}{|c|}{ Vegetables } \\
\hline Category & Mean & $\begin{array}{l}\text { Adj. } \\
\text { Freq }\end{array}$ & Category & Mean & $\begin{array}{l}\text { Adj. } \\
\text { Freq }\end{array}$ & Category & Mean & $\begin{array}{l}\text { Adj. } \\
\text { Freq }\end{array}$ \\
\hline Animal welfare & 6.04 & 39 & Environment & 6.42 & 9 & Check-up & 6.56 & 0 \\
\hline Environment & 5.88 & 34 & Sustainability & 6.36 & 11 & Sustainability & 6.46 & 9 \\
\hline Health & 5.85 & 54 & Check-up & 6.32 & 3 & Honesty & 6.31 & 12 \\
\hline Price & 5.85 & 51 & Honesty & 6.32 & 8 & Additives & 6.27 & 3 \\
\hline Honesty & 5.81 & 19 & Health & 6.29 & 28 & Pesticide use & 6.27 & 61 \\
\hline Pesticide use & 5.81 & 35 & Certification & 6.29 & 7 & Origin & 6.17 & 38 \\
\hline Sustainability & 5.65 & 6 & Quality & 6.25 & 12 & Environment & 6.16 & 35 \\
\hline Quality & 5.58 & 6 & Additives & 6.21 & 14 & Health & 6.15 & 50 \\
\hline Naturalness & 5.52 & 44 & Unprocessed & 6.17 & 9 & Certification & 6.15 & 1 \\
\hline Additives & 5.50 & 19 & Naturalness & 6.00 & 14 & Naturalness & 6.12 & 35 \\
\hline Origin & 5.42 & 19 & Animal welfare & 5.96 & 14 & Unprocessed & 6.00 & 12 \\
\hline Certification & 5.38 & 3 & Origin & 5.86 & 11 & Quality & 5.92 & 11 \\
\hline Lifestyle & 5.27 & 9 & Pesticide use & 5.83 & 5 & Taste & 5.88 & 31 \\
\hline Taste & 5.23 & 20 & Taste & 5.79 & 24 & Nutrition & 5.80 & 15 \\
\hline Check-up & 4.92 & 5 & Lifestyle & 5.74 & 8 & Lifestyle & 5.68 & 4 \\
\hline Nutrition & 4.81 & 6 & Safety & 5.70 & 4 & Availability & 5.60 & 11 \\
\hline Presentation & 4.81 & 3 & Nutrition & 5.70 & 3 & Price & 5.44 & 51 \\
\hline Emotions & 4.69 & 8 & Price & 5.54 & 36 & Safety & 5.44 & 15 \\
\hline Products & 4.65 & 28 & Availability & 5.27 & 4 & Shops/brands & 5.35 & 5 \\
\hline Unprocessed & 4.62 & 14 & Shops/brands & 5.12 & 1 & Animal welfare & 5.20 & 4 \\
\hline Shops/brands & 4.50 & 10 & Scepticism & 5.00 & 28 & Presentation & 5.04 & 4 \\
\hline Safety & 4.50 & 6 & Animals & 4.87 & 5 & Products & 4.92 & 19 \\
\hline Quantity & 4.38 & 4 & Presentation & 4.87 & 7 & Appearance & 4.72 & 20 \\
\hline Appearance & 4.35 & 3 & Emotions & 4.86 & 3 & Scepticism & 4.68 & 11 \\
\hline Trend & 4.23 & 8 & Products & 4.82 & 8 & Emotions & 4.58 & 8 \\
\hline Scepticism & 4.15 & 3 & Quantity & 4.70 & 3 & Quantity & 4.44 & 9 \\
\hline Availability & 4.15 & 9 & Appearance & 4.59 & 1 & Trend & 3.72 & 4 \\
\hline Animals & 3.77 & 3 & Trend & 4.23 & 0 & Animals & 3.63 & 0 \\
\hline
\end{tabular}




\section{Discussion}

Research into organic food has largely focused on consumers' motives to purchase organic food (see Hughner et al., 2007 for review). However, using Construal Level Theory as a psychological approach to understand what consumers think of in relation to the concept "organic" has not been done. Insights in the associations that consumers have provide knowledge about what information consumers need to make wellinformed choices regarding food intake.

Results showed that consumers associated organic overall mostly with "animal welfare", "price", "health", "pesticide use", and "naturalness". Word associations related to "environment", "health", "honesty", "pesticide use", "sustainability", "quality", "naturalness", "additives", "origin", "certification", and "taste" were central across food types (RQ-1). These associations largely correspond to the motives of consumers to purchase organic food (Hughner et al., 2007).

The current research showed that consumers hardly ever mentioned word associations regarding food safety of non-organic food production, which is in contrast with research into motives to buy organic products (Schifferstein \& Ophuis, 1998; Soler et al., 2002). Consumers did mention the "origin" of organic food often, which indicates that the source of food is important. It was expected that consumers would think of support for the local economy (Hughner et al., 2007). Consumers, however, did not mention this aspect. Support for the local economy may not be associated with organic food as such, but instead concern the overall practice of organic food production.

Price was frequently mentioned, which is in line with research into the motives not to purchase organic food (Lea \& Worsley, 2005; Magnusson et al., 2001; Zanoli \& Naspetti, 2002). It was, however, perceived as peripheral to organic meat and organic vegetables. This implies that consumers view organic food as expensive, but when thinking of more narrow food categories, other characteristics are perceived as more important than price.

Our results, both word associations and spatial psychological distance scores, showed that all consumers groups perceived organic food to be close by as opposed to unavailable. Zanoli \& Naspetti (2002) found that lack of availability was an important motive not to buy organic food. This seems to be less prominent in our study. A possible explanation could be that organic food has become more available the last few years in Europe (Giraud, 2002). In the Netherlands the sales of organic food increased with $10 \%$ in 2014 and organic food is largely sold through supermarkets and mainstream outlets nowadays (FoodHolland, 2016).

Following Construal Level Theory (Trope \& Liberman, 2010) it was predicted that organic food would bring to mind more abstract and distant word associations than 
organic meat or organic vegetables (RQ-2). Such a difference was not found: looking at the word associations, the abstractness (e.g. inclusion of details) was equivalent. However, there was dissimilarity in the centrality ratings, which suggest that consumers did have more concrete word associations regarding organic meat or organic vegetables compared to organic food. The centrality rating for organic food was overall lower than organic meat or organic vegetables. As a low centrality rating implies that the word associations were less central to the concept and thus more psychological distant, this result corresponds to our predictions based on Construal Level Theory.

Consumers were observed to be more sceptical of organic meat than organic vegetables (RQ-2). Word associations regarding meat showed a concern with animal welfare. Word associations with vegetables, in contrast, indicated that consumers were concerned with human health. This implies that when authorities want to promote organic products, they should adapt their information supply in a way that they focus on health aspects when promoting vegetables and on animal welfare when promoting meat.

Differences in word associations between consumer groups (frequently, occasionally, hardly ever buyers) were found (RQ-3). Overall, frequent consumers were more positive about the concept of organic foods and seemed to be less concerned about negative aspects, such as a high price. This supports the finding by Harper and Makatouni (2002). It was also reflected in the values regarding organic food. Consumers who frequently buy organic showed the strongest values regarding environment, animals, and human health, while they valued price the least. This is consistent with Zanoli \& Naspetti (2002), who found that occasional buyers lacked altruistic values that frequent consumers possessed. It suggests that values are important in predicting organic food purchasing.

As expected, psychological distance was smallest for frequent buyers and greatest for consumers who hardly ever purchase organic food (RQ-4). The relationship between psychological distance and purchase behaviour is yet unclear. One could argue that the attractiveness of potential options, and consequently consumers' food decisions, depends on the distance to them (Fiedler, 2007; Trope et al., 2007): close objects are preferred over distant objects. Higher levels of psychological distance are thus likely to be related to non-buying. Another option, however, would be that non-buying increases psychological distance. Policy makers or advertising agencies, for instance, thus can make use of the mechanism of psychological distance and induce closeness instead of distance to influence purchase behaviour. They may, for example, decrease affective distance by emphasizing the consumers' fit with the product and emphasizing similarities between organic food consumers and the consumer at hand. 
The results of the assessment of the socio-demographic characteristics (RQ-4) showed that the consumer groups differed in age and gender, indicating that older people were more likely to be consumers of organic food, as were women (Lea \& Worsley, 2005). Gender and age differences might be a reflection of a different prioritisation in how to spend money and time. It might be that men in our sample were less often responsible for doing the grocery shopping than women and are therefore less concerned with or less informed about food, which was also reflected in their higher hypothetical, affective and informational distance compared to women.

A strength of our research lies in the distribution of the consumer groups in study 2.1. Our sample included, not only consumers who frequently buy organic food, but also consumers who occasionally or hardly ever purchase organic products and thus provides information about word associations across different consumer groups. It remains, however, unclear to what extent our results are generalizable to the European population and how specific subpopulations, such as mothers with young children or individuals with chronic health issues, think of eating organic food. For these individuals health is particularly relevant, which may impact the associations present. It might be that their associations are more focused around health-aspects, while associations such as animal welfare and availability, might be less prominent. In addition, values might be strongest regarding human health compared to animals, environment, price and attractiveness. Further research is needed to examine these relationships for specific subpopulations.

Taken together, in this research we established a framework of the word associations of organic food by consumers with different purchase behaviours. The insights established in this framework showed that information supply focused on promoting organic food should be tailored depending on both food type and consumer group. 


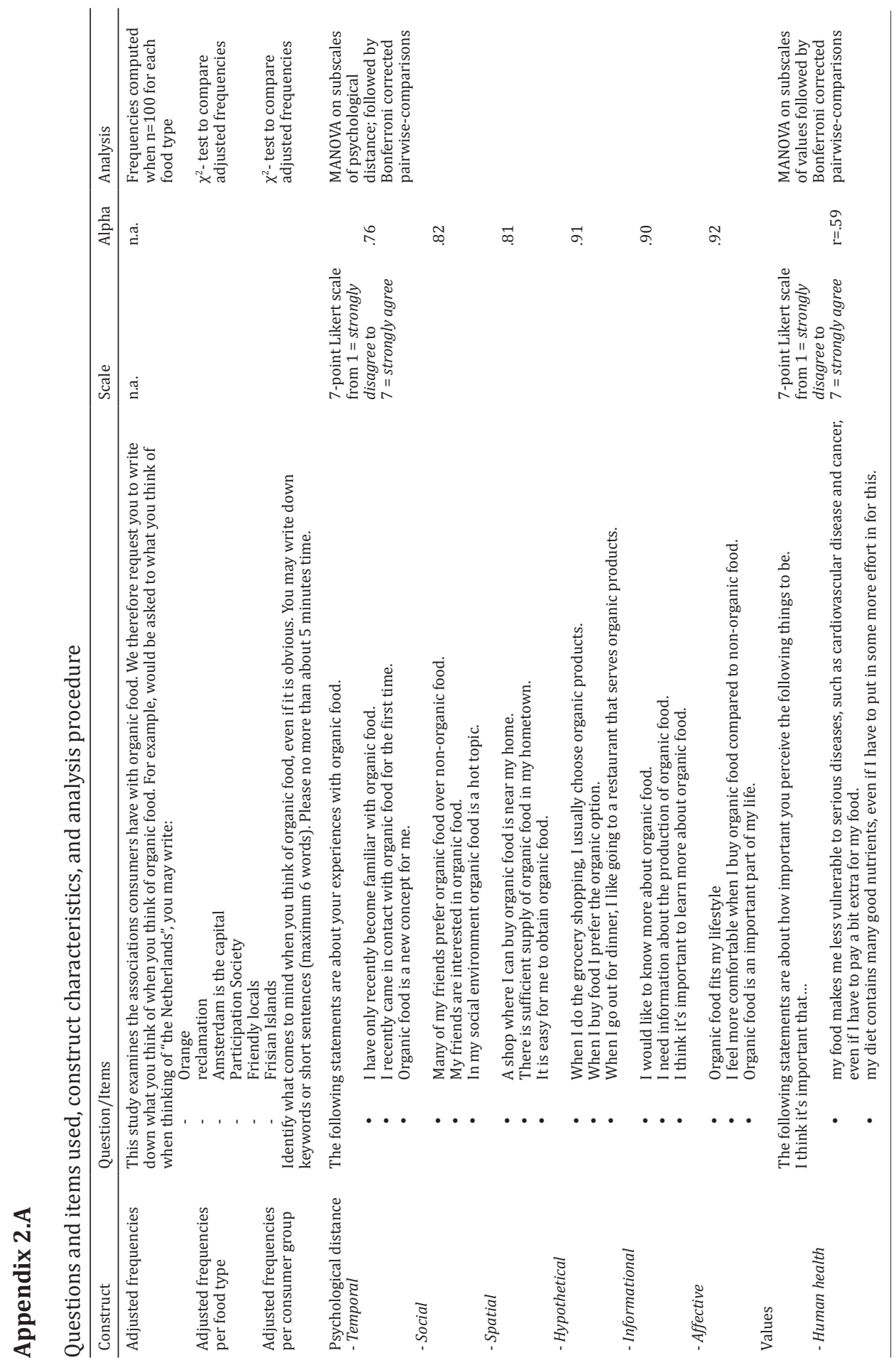




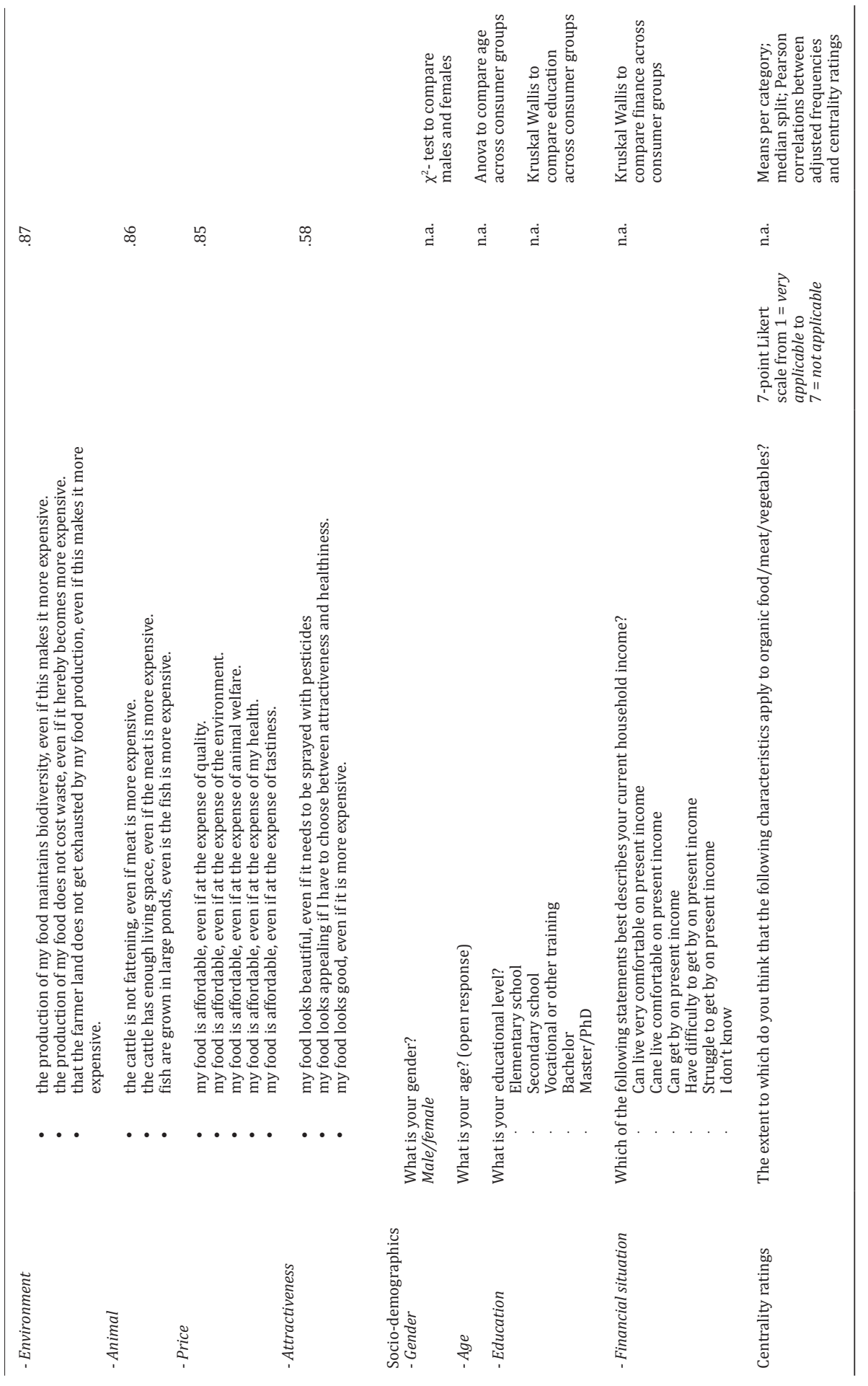




\section{Appendix 2.B}

Categories of associations

\begin{tabular}{|c|c|}
\hline Category & Content \\
\hline Additives & $\begin{array}{l}\text { Less or no chemical or artificial additives, no added e-numbers, no artificial } \\
\text { flavouring, no added sugar. }\end{array}$ \\
\hline Animal welfare & Good treatment of animals. \\
\hline Animals & Specific types of animals. \\
\hline Appearance & Colourful, imperfect shapes. \\
\hline Availability $^{*}$ & Variation in offerings, ease of obtainability. \\
\hline Certification $^{* *}$ & Specific forms of certification, presentation of certification. \\
\hline Check-up & Check-ups, verification difficulties, quality control, legislation and demands. \\
\hline Emotions & Positive emotions/feelings, happiness, friendliness, production with love. \\
\hline Environment $^{* * *}$ & Better for the environment/earth/animals, healthy soil, attention to nature. \\
\hline Health $^{* * *}$ & Healthy, healthier \\
\hline Honesty & Responsibility, fairness, ethics, conscientiousness, honesty. \\
\hline Lifestyle & Normality of eating organic food, the type of people that eat organic food. \\
\hline Medicine & A lack or less antibiotics use, medicine or hormones. \\
\hline Naturalness & Natural, pure, clean, green, fresh, real. \\
\hline Nutrition & Nutritious, containing more vitamins, more minerals. \\
\hline Origin & $\begin{array}{l}\text { Farms, gardens, agricultural references, soil, place of production, cultivation } \\
\text { in line with natural processes. }\end{array}$ \\
\hline Pesticide use & A lack or less pesticide use, use of natural sprays. \\
\hline Presentation & Packaging, marketing, presentation in media. \\
\hline Price $^{* * * * *}$ & Expensive, more expensive than 'regular' food. \\
\hline Products & Specific types of food products. \\
\hline Quality ${ }^{* * * * * *}$ & $\begin{array}{l}\text { Quality, organic food being better in general, better preserving, more fresh, } \\
\text { one gets more for the money. }\end{array}$ \\
\hline Quantity & $\begin{array}{l}\text { Quantity of food being consumed, eating less meat and more vegetables, } \\
\text { smaller size of the production establishments, more loss of harvest, smaller } \\
\text { profit. }\end{array}$ \\
\hline Safety & Safer, not or less poisonous substances. \\
\hline Scepticism & Doubts about organic food, negative aspects to organic food. \\
\hline Shops/brands & Specific organic brands, (web)shops or places. \\
\hline Sustainability ${ }^{* * * * * * *}$ & Sustainability, agricultural cycle, less future risks. \\
\hline Taste & Tasty or tastier, better flavour. \\
\hline Trend & Popularity, trending topic. \\
\hline Unprocessed & Unprocessed food, not manipulated/modified. \\
\hline
\end{tabular}

*Two associations refer to unavailability.

${ }^{* *}$ One association refers to fake certificates.

${ }^{* * *}$ One association refers to meat putting demands on the environment.

${ }^{* * * *}$ One association refers to being unhealthy.

${ }^{* * * * *}$ One association refers to being affordable.

${ }^{* * * * * *}$ Two associations refer to being more likely to spoil.

${ }^{* * * * * * *}$ One association refers to unsustainability. 
THE EFFECT OF ONLINE SOCIAL PROOF REGARDING ORGANIC FOOD

Comments and Likes on Facebook

This chapter is based on: Hilverda, F., Kuttschreuter, M., \& Giebels, E. (submitted). Comments and Likes on Facebook: The Effect of Online Social Proof 
The rise of social media provides new opportunities to non-profit organisations (Galvez-Rodriguez et al., 2016), and more specific to food risk communicators (Rutsaert et al., 2013b; Rutsaert et al., 2014). Advantages of using social media to disseminate information are, for instance, speed and accessibility (Rutsaert et al., 2014). Social media also empower individuals to interact with other consumers and express their own opinion (Shao, 2009), resulting in an increase of public involvement and interaction (Rutsaert et al., 2013b). This raises the question to what extent individuals are influenced by online cues that signal the views of others with respect to food risk issues, such as online comments and the number of likes at a statement.

Generally, the Internet is becoming one of the main sources of information (Jacob et al., 2010; Kuttschreuter et al., 2014; Redmond \& Griffith, 2006; Tian \& Robinson, 2008). In the early days, the Internet was primarily used to search for and check information. Nowadays it has changed into a dynamic information environment of social media, where basically everyone can post messages, and spread or comment on information rapidly (Horst et al., 2007). This results in an abundance of facts and viewpoints on a particular topic, which might be helpful as well as confuse Internet users. Individuals have to make sense of this information and consequently decide how to act.

When searching for information on the Internet, it is quite possible that individuals consult a social media website including Facebook and Twitter (Giustini, 2006), rather than a more traditional website of an official information body (Rutsaert et al., 2013a; Rutsaert et al., 2013b). According to Cialdini's principle of social proof (Cialdini, 2001), in ambiguous situations where individuals are uncertain about an appropriate course of action, they may assimilate to the viewpoints and behaviours of (similar) others who might be more knowledgeable in dealing with the particular situation. Social media information might serve this purpose.

The current research is conducted in the Netherlands, where already back in 2012, nearly $70 \%$ of the Dutch Internet users used social media (CBS, 2013). Facebook, the most popular social media channel (Cheung et al., 2011), is one of the main means of communication and gained popularity in the Netherlands the last years. Facebook members use Facebook to spread (personal) information to their friends or to, depending on their privacy settings, a broader audience (Kirschner, 2015). Individuals can post a statement or message on Facebook (a post), respond to these posts (the comments) and indicate that they agree with the post and/or comment by using the "thumb up" symbol (the likes). Facebook usage is not only associated with interpersonal communication and maintaining relationships (Cheung et al., 2011), individuals also use Facebook to seek and respond to information (Basilisco \& Cha, 2015). In particular, individuals search on Facebook for consumer trends information (Asghar, 2015). Increasingly, Food communicating organisations (e.g. the Netherlands Food and Consumer Product Safety 
Authority and the Netherlands Nutrition Centre Foundation) respond to this by using Facebook to communicate with individuals and inform them about (food) issues.

This research aims to investigate to what extent individuals are susceptible to social proof in an online environment. In two experimental studies involving a fictitious Facebook page on organic food products, we manipulated social proof by varying the comment valence and the number of likes, and examined the effect on perceptions, feelings and intended purchasing behaviour. We examined the research question: "To what extent do comments and likes on Facebook influence individuals' perceptions, feelings and behaviour towards organic food?"

\section{Online Social Proof: Comments, Narratives and other explicit cues}

According to Cialdini (2001), one of the most influential social influence mechanisms is the principle of social proof. This principle is based on the heuristic that individuals follow the lead of (similar) others (Cialdini, 2001; Okdie et al., 2013). Basically, the idea is that, when many individuals comply with a certain course of action, the risk of making a wrong decision decreases (Lee et al., 2008).

As the Internet is becoming more popular, new forms of social proof arise. For example, there are reviews, narratives, personal blogs, and opinion pages available online. These can be used as indicators of the way in which similar others perceive a certain topic and can in principle create, change or adjust opinions. One of the key aspects of online social proof is its valence: positivity (being pro) versus negativity (being against).

Several studies have shown the impact of positive and negative online reactions of other consumers on behaviour (Winterbottom et al., 2008) and attitudes (Vermeulen \& Seegers, 2009). Vermeulen and Seegers (2009), for example, investigated the effects of online hotel reviews on attitudes towards hotels. While both positive and negative reviews increased awareness of specific hotels, and - not surprisingly - they found that positive reviews were associated with more favourable attitudes towards hotels than negative reviews.

Although the impact of viewing solely negative or solely positive online social proof has been studied extensively, in reality it is more likely that individuals are exposed to both positive and negative opinions from different sources at the same time (Lee et al., 2008). In line with this presumption, a study on vaccination focused on the proportion of online narratives (peer comments) that reported adverse consequences (Betsch et al., 2011). They observed that the higher the percentage of negative narratives, the more the risk perception increased, which in turn led to a lower intention to vaccinate.

Building on these results, Kause et al. (2014) performed a study in which they systematically varied the proportion of negative and positive narratives about flu 
vaccination (ratio of positive comments: $0,0.25,0.50,0.75$, and 1.00 ). Results showed only one significant post-hoc effect between conditions: the intention to vaccinate differed between the participants who received only negative comments and those who received only positive ones. That is, the participants who viewed only positive comments were more inclined to vaccinate compared to individuals who viewed only negative comments. Similar results were obtained in a recent study by Seo et al. (2015) who examined the effect of Facebook comments on food safety information regarding restaurants. With the proportion of positive comments varying between $0,0.50$ and 1.00 , this study showed that the higher the proportion of positive comments, the lower the level of risk perception related to eating in the advertised restaurant.

Similar studies have been done to examine the effect of online social proof by means of Facebook comments in different contexts, varying from breastfeeding attitudes (Jin et al., 2015) to marihuana legalization (Winter et al., 2015), and brand engagement and sales (Kim \& Johnson, 2016). The empirical evidence for the effect of (online) social proof is, however, not restricted to Facebook. Studies have also shown the effect of social proof on YouTube in relation to smoking behaviour (Shi et al., 2014; Walther et al., 2010).

Regardless of the specific context or type of online social proof, it remains unclear what happens when positive and negative comments are presented together in a way that makes the information in the comments inconclusive. This is, for example, the case when there are as many individuals who are in favour of a particular activity as individuals who are against it. When individuals cannot draw conclusions from explicit cues of online social proof, such as the comments, they might start to look at other pieces of information, such as the number of likes.

\section{Subtle cues of online social proof}

When explicit expressions of social proof such as comments are unavailable or contradictory, or when cognitive resources are limited, individuals might look at more subtle cues of social proof. Research supports this idea. Amblee and Bui (2011) conducted a study on online reviews of short e-books. They showed that individuals focused their attention on the book reviews rather than on the author ratings. When there were no reviews available, however, individuals switched their attention towards the author ratings. This thus suggests that when explicit cues are missing, individuals might turn to other, more subtle cues.

This effect may hold for other implicit cues of social proof, such as the number of likes at Facebook comments and the number of downloads on iTunes. Individuals may view these subtle cues as endorsement: when there are many likes at a statement, the statement might be perceived as more valid because many individuals clicked the like button and thus agreed with it. 
Previous research into the effect of likes on Facebook showed mixed results. Jin et al. (2015) found evidence for the importance of the number of likes on pro-breastfeeding attitudes. In contrast, other research showed that the subtle cues of the number of likes of a comment did not influence individuals' attitudes (Peter et al., 2014; Winter et al., 2015). These studies used the exemplification theory (Zillmann, 2002) to explain the insignificant effect of the number of likes: Individuals are more easily influenced by exemplifying statements than user statistics, such as the number of likes. While Peter et al. (2014) did not find an effect of comment likes, their results indicated an effect of post likes on attitude towards flue vaccination. Concluding, there is some evidence indicating that likes are perceived as endorsement of posts and comments, but results are inconclusive.

\section{Social proof by social media and organic food products}

An open question is whether social proof is effective in the case of organically grown food products. The supply of these products is growing, and individuals have to make up their minds regarding purchasing and consuming those products. This decision is not that simple, however, as it involves the weighing of the risks and benefits of such products from a health as well as environmental perspective. Following the social proof principle, individuals might be inclined to follow the lead of similar others in this situation.

Organic food products match a general preference for naturalness and foods produced without human intervention (Rozin et al., 2004; Shafie \& Rennie, 2012). Research shows that organic agriculture is generally perceived as quite positive (Magnusson et al., 2001; Saba \& Messina, 2003) and that consumers consider organic foods to have advantages over conventional foods, such as higher quality and better taste (Grankvist \& Biel, 2001, 2007; Hay, 1989; Roitner-Schobesberger et al., 2008; Schifferstein \& Ophuis, 1998). They also perceive organic foods to be less risky than conventional food (Hammitt, 1990). Specifically, the risk of microbiological contamination and natural toxins is considered to be very small compared to the risks of pesticide use (Williams \& Hammitt, 2001).

At the same time, the very absence of pesticides makes these products more prone to bacterial contamination. This might induce food risk communicators to advice the general public on such risks, which might create uncertainty among individuals and make them susceptible to social proof. This raises the question, to what extent individuals are influenced by online cues that signal the views of others with respect to food risk issues, such as online comments and the number of likes at a statement. 


\section{Evaluation of the comments}

When testing effects of comment valence on attitudes, it is important to take the individual's evaluation of the comments into account. Slater and Rouner (1996) argue that the evaluation of a message has an effect on source credibility and in turn influences message acceptance and belief change. A more recent study (Lee \& Shin, 2014) showed that the quality of online reviews had an impact on product evaluations and purchase intentions. This could mean that individuals who perceive the comments to be clearer and more valuable are more likely to be influenced by explicit online social proof in a way that positive Facebook comments lead to more positive reactions, while negative comments lead to negative reactions.

\section{The current studies}

Based on the previous discussion we examined explicit and implicit ways of online social proof on Facebook pages, namely the comments'valence (positive versus negative) and the number of likes (high versus low) placed below these comments. In study 3.1, we examined the effect of these types of online social proof on perceptions, feelings and behaviour towards organic food, predicting that individuals were more easily influenced by comments than by the number of likes. The evaluation of the comments was included as a moderator.

As it is more likely that, in the real world, individuals are exposed to both positive and negative opinions from different sources at the same time (Lee et al., 2008), in study 3.2 we examined the effect of the number of likes on a mixed valence Facebook page.

\section{Study 3.1: The interplay of comments and likes on Facebook}

\section{Hypotheses}

In study 3.1 we tested to what extent individuals were influenced by both comments as well as likes on Facebook. In this 2x2 study, the valence of the comments (positive versus negative) and the reinforcement (high versus low number of likes) were manipulated.

It was first examined whether the levels of comment valence affected perceptions, feelings and behavioural intentions. It was predicted that:

H1: There is a main effect of comment valence in a way that positive comments lead to more positive perceptions, feelings, and behavioural intentions towards organic produce compared to negative comments. 
Subsequently, the effects of the number of likes and the interaction between valance and number of likes were examined. We hypothesized that:

H2: There is no significant main effect of reinforcement: The number of likes does not have an effect on the dependent variables.

H3: There is an interaction effect of valence and reinforcement: The number of likes strengthens the effect of the comments.

In addition, the evaluation of the comments was included as a moderator predicting that:

H4: There is a main effect of evaluation of the comments on the dependent variables.

H5: There is an interaction effect of the evaluation of the comments and comment valence in a way that positive comments lead to more positive perceptions, feelings, and behavioural intentions towards organic produce and negative comments to more negative reactions, especially when the comments are perceived to be clear and useful.

\section{Method}

Participants. A total of 241 participants recruited by a research agency completed an online experiment, which took them about 15 minutes. The research agency acted in accordance with the ethical standards of the institutional research committee. The sample was representative of the Dutch population of Internet users with respect to age and gender. Only participants who correctly filled out both manipulation check questions were included in the analyses ${ }^{2}$. This resulted in a total sample of 124 participants. There were no differences with respect to age and gender between the final sample and the drop-outs.

Participants were 48 years old on average $(S D=16.62)$. The sample consisted of 65 males (52\%) and 59 females (48\%). All of them were familiar with Facebook. A randomization check showed that there were no differences between condition with respect to gender, age, and initial knowledge, and initial risk and benefit perception of organic food.

Design and Manipulation. We used a 2 (comment valence: positive vs negative) x 2 (reinforcement: high vs low number of likes) between subjects design to test the hypotheses. Participants were randomly assigned to one of the four conditions $\left(\mathrm{n}_{\text {positive- }}\right.$ high $=44, \mathrm{n}_{\text {positive-low }}=18, \mathrm{n}_{\text {negative-high }}=47$ and $\mathrm{n}_{\text {positive-low }}=15$ ).

\footnotetext{
${ }^{2}$ A total of $97 \%$ participants correctly filled out the manipulation check question about the valence of the comments ( $92 \%$ in the positive condition and $94 \%$ in the negative condition). A total of $52 \%$ participants correctly filled out the manipulation check question about the number of likes $(76 \%$ in the many likes condition and $28 \%$ in the few likes condition).
} 
Participants viewed a Facebook image with four comments (see appendix 3.A). The valence of these comments varied. A pilot study was conducted among students to select comments that were identical content-wise while different with respect to valence (positively formulated comments and similar negatively formulated comments). The comments with the largest contrast were selected, provided the participants perceived the comments to be realistic Facebook statements.

Comment valence was manipulated by varying the content of the comments: in the positive condition all comments were positive; in the negative condition all comments were negative. Reinforcement was manipulated by varying the number of likes: either a high or low number of likes was given to the comments.

\section{Measures.}

Perceptions. Three measures of perceptions were used. To measure personal health risk perception, participants indicated, whether they considered eating organic to be unhealthy ( 4 items; $\alpha=.93 ; 7$-point-Likert scale; $1=$ strongly disagree to $7=$ strongly agree). We measured benefit perception regarding one's own health with four statements about eating organic food being healthy (4 items; $\alpha=.90$, 7-point-Likert scale, 1 = strongly disagree to 7 = strongly agree). Participants also indicated on a 7-point semantic differential-type scale (Osgood et al., 1957) what their overall attitude of eating organic was. Three item-pairs were used $(\alpha=.82)$ : negative-positive, bad-good, and bad for my health-beneficial for my health.

Feelings. We measured emotions by asking participants to what extent they experienced anxiety (anxious, concerned, afraid and worried; $\alpha=.94$ ) and positive emotions (happy, positive, satisfied, optimistic; $\alpha=.97$ ) when thinking about eating organic food. Items were measured on a 7-point scale from 1 = not at all to 7= very much .

Behavioural predictors. Two behavioural predictors were measured: motivation to find information and willingness to buy. Participants filled out whether they wanted to know more about organic food (4 items; $\alpha=91$; 7-point scale; $1=$ strongly disagree to 7 = strongly agree). As research showed that consumer attitudes are more reliably measured and more predictive of behaviour, when focusing on specific food products rather than broad product categories (Bredahl, 1999), willingness to buy was measured by asking the participants to what extent they were inclined to buy 7 organic food products (7-point scale, ranging from $1=$ not at all to $7=$ very much; adapted from Makatouni, 2002; $\alpha=.96)$.

Comment evaluation. We measured participants' evaluation of the Facebook comments with three statements about their clearness, usefulness in contribution to an advice for a friend and whether they discussed important aspects of organic products (3 items; $\alpha=.67,7$-point-Likert scale, $1=$ strongly disagree to 7 = strongly agree). 
Procedure. Participants were requested to fill out an online questionnaire about new food production methods. A request by a friend for advice on purchasing organic food was used as a cover story to increase involvement. After filling out the questions about their initial knowledge and perceptions regarding organic food, participants viewed the Facebook image. The participants, then, indicated what they thought about the discussion on Facebook, and filled out the two manipulation check questions. The dependent variables were then measured. At the end, the participants answered questions about their socio-demographics and their online media use, and were thanked for their participation. During the whole data collection period, participants could contact the helpdesk of the research agency for questions and debriefing.

\section{Results study 3.1}

Means and correlations. Table 3.1 reports the means, standard deviation, reliabilities and correlations of the constructs of the study. Risk perception and anxiety were relatively low, while benefit perception and attitude were quite high. Positive emotions, motivation to find information, willingness to buy and the evaluation of the comments scored all somewhat above the midpoint of the scale.

Risk perception correlated positively with anxiety and negative with benefit perception, attitude, positive emotions, and willingness to buy. The opposite was true for benefit perception: Higher benefit perceptions were associated with less risk perception and anxiety and higher levels of attitude, positive emotions and willingness to buy. While benefit perception was positively related to motivation to find information, risk perception was unrelated to this construct. Attitude and positive emotions showed similar correlation patterns as benefit perception, though the correlations between attitude and motivation to find information, and anxiety and positive emotions were not significant. Anxiety was negatively correlated with willingness to buy; the more anxiety, the less willing to buy. Only the motivation to find information was significantly related to the evaluation of the comments.

Hypotheses testing. To test the hypotheses a GLM was performed to test the effect of valence, the number of likes and their interaction on the six dependent variables at once, including evaluation of the comments as moderator (centred around the mean). This GLM was followed by a separate ANCOVA for each of the dependent variables.

There were no statistically significant multivariate effects of valence (H1), likes $(\mathrm{H} 2)$, nor the interaction between valence and the number of likes (H3), all $p$ 's $>.05$. These results are in contrast with $\mathrm{H} 1$ and $\mathrm{H} 3$ that predicted a significant main effect of valence and an interaction effect with this effect strengthened by the number of likes. The absence of a main effect of the number of likes, is in line with H2. 
Table 3.1 Means, standard deviations, reliabilities, and correlations between all constructs (n =124)

\begin{tabular}{|c|c|c|c|c|c|c|c|c|c|c|c|}
\hline \multicolumn{2}{|c|}{ Constructs } & \multirow{3}{*}{$\begin{array}{l}\text { Mean } \\
2.76\end{array}$} & \multirow{3}{*}{$\begin{array}{l}s d \\
1.14\end{array}$} & \multirow{3}{*}{$\begin{array}{l}\alpha \\
.93\end{array}$} & \multicolumn{2}{|c|}{ Correlations } & \multirow[b]{2}{*}{3.} & \multirow[b]{2}{*}{4.} & \multirow[b]{2}{*}{5.} & \multirow[b]{2}{*}{6.} & \multirow[b]{2}{*}{7.} \\
\hline & & & & & 1. & 2. & & & & & \\
\hline 1. & $\begin{array}{l}\text { Personal health risk } \\
\text { perception }\end{array}$ & & & & & & & & & & \\
\hline 2. & $\begin{array}{l}\text { Personal health benefit } \\
\text { perception }\end{array}$ & 4.80 & 1.02 & .90 & $-.66^{* *}$ & & & & & & \\
\hline 3. & Attitude & 4.78 & 1.33 & .82 & $-.52 * *$ & $.66^{* *}$ & & & & & \\
\hline 4. & Anxiety & 2.02 & 1.21 & .94 & $.50^{* *}$ & $-.37 * *$ & $-.33^{* *}$ & & & & \\
\hline 5. & Positive emotions & 4.14 & 1.68 & .97 & $-.46^{* *}$ & $.68^{* *}$ & $.55^{* *}$ & -.13 & & & \\
\hline 6. & $\begin{array}{l}\text { Motivation to find } \\
\text { information }\end{array}$ & 4.45 & 1.24 & .91 & .02 & $.35^{* *}$ & .14 & .001 & $.29 * *$ & & \\
\hline 7. & Willingness to buy & 4.20 & 1.65 & .96 & $-.48^{* *}$ & $.73^{* *}$ & $.60^{* *}$ & $-.22^{*}$ & $.70^{* *}$ & $.41^{* *}$ & \\
\hline 8. & Evaluation comments & 4.22 & 1.09 & .67 & .04 & .14 & -.05 & .01 & .05 & $.27^{* *}$ & .11 \\
\hline
\end{tabular}

**. Correlation is significant at the 0.01 level (2-tailed); *. Correlation is significant at the 0.05 level (2-tailed).

Note: All constructs were measured on a 7-point Likert scale

There was a significant multivariate effect of the evaluation of the Facebook comments (H4), $F(7,112)=3.55, p=.002$; Wilk's $\lambda=0.82$, partial $\eta^{2}=.18$. Univariate analysis showed that this effect only held for benefit perception, $F(1,118)=7.21, p=$ .008 , and motivation to find information, $F(1,118)=14.40, p<.001$, showing that the more clear and useful the comments were perceived, the more benefits participants perceived and the more motivated they were to find additional information, confirming hypothesis 4 . The multivariate interaction of valence and the evaluation of the comments (H5) was also significant, $F(7,112)=5.60, p<.001$; Wilk's $\lambda=0.74$, partial $\eta^{2}=.26$. Univariate analysis showed that this effect held for all dependent variables: risk perception, $F(1,118)=7.29, p=.008$, benefit perception, $F(1,118)=20.21, p<.001$, attitude, $F(1,118)=6.13, p=.015$, anxiety, $F(1,118)=13.87, p<.001$, positive emotions, $F(1,118)=14.28, p<.001$, motivation to find information, $F(1,118)=8.09, p=.005$, and willingness to buy, $F(1,118)=5.66, p=.019$. Figure 3.1 shows the interaction effect of valence and comment evaluation for risk perception. This figure shows that the higher the evaluation of the comments, the stronger the effect of social proof in the expected direction. This pattern is similar for anxiety and reversed for the other dependent variables. These results are in line with hypothesis 5 and confirm the moderating role of comment evaluation. Please see Table 3.2 with the means per condition, including corresponding $F$ - and $p$-values. 


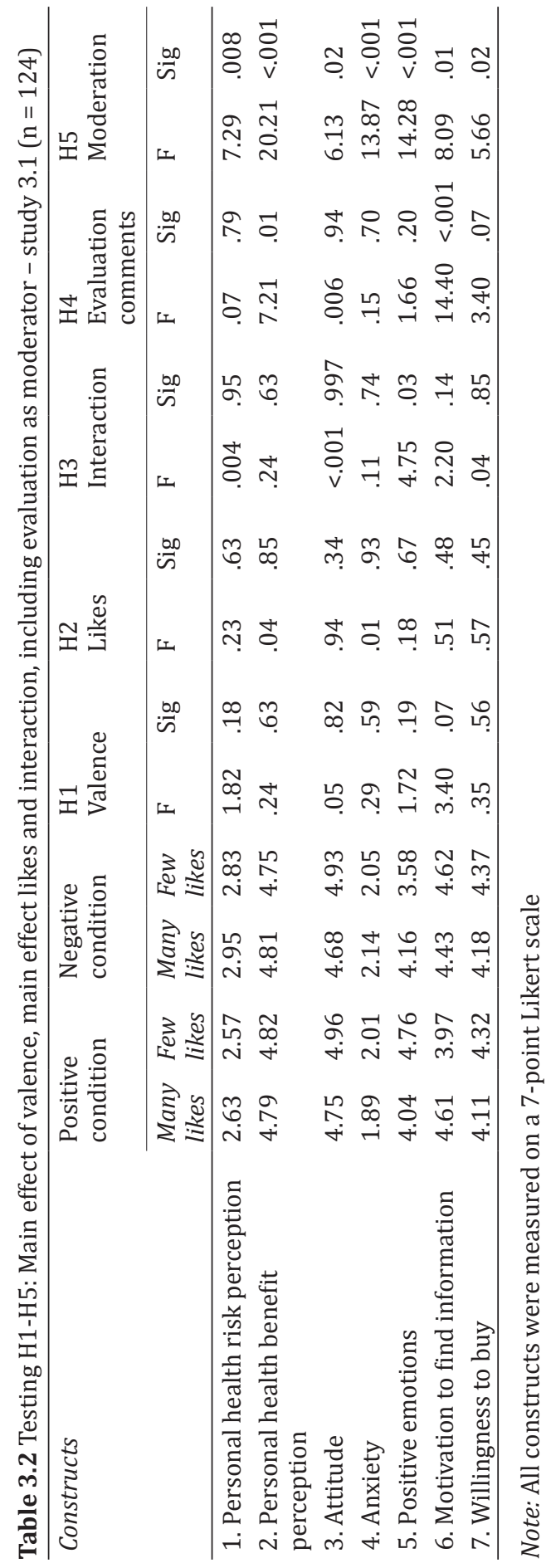




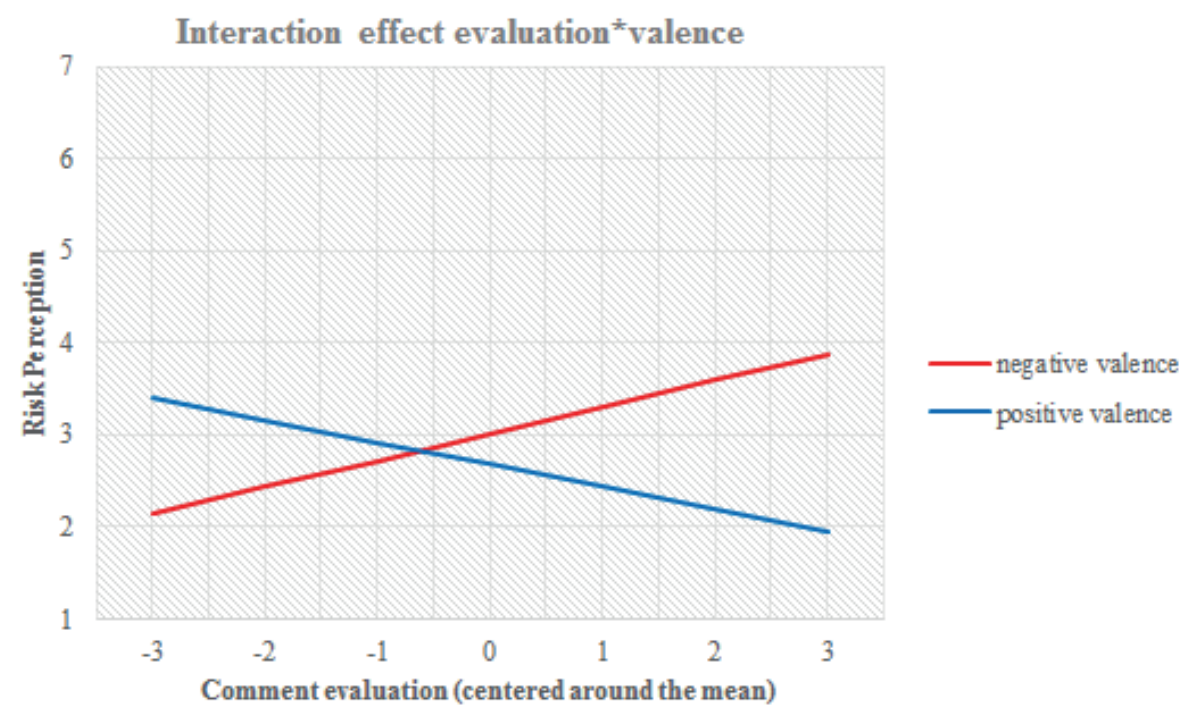

Figure 3.1 Interaction effect of comment evaluation and valence on risk perception.

\section{Study 3.2: The full Facebook page}

In study 3.2 we focus on the effect of the number of Facebook likes when individuals are exposed to both positive and negative opinions from different sources at the same time. The participants were shown a fake Facebook page with multiple posts and comments about eating organic foods, in which either the positive or the negative statements are reinforced by a high number of likes. Positive comments stress that the absence of pesticides (which are used in traditional agriculture) improves the healthiness of organic food, while negative comments emphasize that organic foods are not always pesticide-free and that consumers underestimate the risks of organic products, such as bacterial contamination. We expect that participants in the reinforcement of positive comments condition are overall more positive about organic products than participants in the negative reinforcement condition. The following hypotheses are tested: Reinforcement of positive comments (compared to negative comments) leads to:

H1: More positive perception of organic food:

a) a higher perception of the benefits of organic food products

b) a higher perception of pesticide risks

H2: More positive feelings:

a) more positive emotions

b) less anxiety 
H3:Higher behavioural intentions towards organic food:
a) a higher motivation to find more information about organic products
b) a higher willingness to pay for an organic apple compared to a regular apple

\section{Method}

Design and Participants. There were two conditions with the same statements and comments. In the reinforcement of positive comments condition, many likes were given to positive statements regarding organic foods and few likes to negative. In the negative condition, this pattern was reversed: many likes were given to negative statements and few likes to positive statements. Participants were randomly assigned to one of the two conditions.

This study was performed in accordance with the ethical standards of the institutional research committee. Participants were psychology students who participated to earn course credits. They were requested to fill out an online questionnaire, which took them about 15 minutes in total. The final sample consisted of 88 participants $^{3}$. This sample consisted of 25 males (28\%) and 63 females (72\%) who were 21 years old on average. A randomization check showed that there were no differences between condition with respect to gender and age.

Measures. Instruments were similar to those in study 3.1, except for a few modifications ${ }^{4}$.

Perceptions. There were two perception measures. To measure benefit perception $(\alpha=.86)$, participants filled out to what extent they agreed that organic products have advantages (for example "more nutritious"). The scale consisted of 12 items, that were all measured on a five-point-Likert scale $(1=$ strongly disagree to $5=$ strongly agree $)$. To measure risk perception of pesticide use, participants indicated on a 5-point scale, ranging from $1=$ strongly disagree to $5=$ strongly agree, to what extent they thought pesticides were harmful to humans (3 items), animals (3 items), and the environment (3 items). Reliability was good $(\alpha=.94)$.

\footnotetext{
${ }^{3} \mathrm{~A}$ total of 88 participants (out of 145 ) correctly filled out the manipulation check directly after viewing the Facebook image; they were aware that there was more support (number of likes) in favour of eating organic foods in the positive condition, respectively against eating organic foods in the negative condition. Only those participants who correctly answered the manipulation check variables were included in the analyses.

${ }^{4}$ All constructs in study 3.2 were measured on a 5-point Likert scale instead of a 7-point scale. As a replacement for health risk perception of eating organic food we measured risk perception of pesticide use, which was found to be a central association with respect to organic food (Hilverda, Jurgens \& Kuttschreuter, 2016). Attitude was excluded to shorten the questionnaire. With respect to behavior, willingness to pay was used as a substitute for willingness to buy as how much money a respondent is willing to pay is expected to be more closely linked to actual behavior than buying intentions.
} 
Feelings. We measured positive emotions by asking to what extent they experienced four positive states (happy, positive, satisfied, being in a good mood; $\alpha=.94$ ). Items were measured on a 5 -point scale from $1=$ not at all to $5=$ very much. Analogously, we measured anxiety by asking the participants to what extent they experienced negative emotions when thinking about eating organic food (anxious, concerned, hopeless, pessimistic; $\alpha=.80$ ).

Behavioural predictors. Two measures were used to measure predictors of behaviour towards eating organic: the motivation to find information and the willingness to pay. Participants filled out, on a 5-point scale ranging from $1=$ strongly disagree to 5 = strongly agree, whether they wanted to know more about organic food (4 items; $\alpha=$ .77). Participants were then asked how much they were willing the pay for an organic and a regular apple. The difference in eurocents in the amount of money was used in the analysis.

Procedure. Participants were requested to fill out an online questionnaire about organic food. After filling out an informed consent form and their socio-demographics, participants viewed an image of a Facebook page with several comments. They then answered an open-ended question about their opinion of the information on the Facebook page, and filled out the manipulation check. After that, the dependent variables were measured. Participants were debriefed by email afterwards.

\section{Results study 3.2}

Means and correlations. Please see Table 3.3 for means, standard deviation, reliabilities and correlations of the constructs used in the study. Risk perception was relatively high. Benefit perception and positive emotions were around the mid-point of the scale. They correlated positively with each other and with the perceived risks of pesticide use. Higher benefit perceptions were associated with more positive emotions and higher risk perception of pesticide use. Anxiety was low and did not correlate with any of the other dependent variables. There was a positive correlation between motivation to find information and positive emotions, meaning that more positive emotions were related to a higher motivation to find information.

On average, participants were willing to pay 19 cent more for an organic apple than for a regular apple. This amount was related to risk perception of pesticide use and positive emotions towards eating organic food: the higher the perceived risks of pesticide use and the more positive emotions with respect to eating organic, the higher the amount the participants were willing to pay. 
Table 3.3 Means, standard deviation, reliabilities, and correlations - study 3.2 ( $\mathrm{n}=82-88)$

\begin{tabular}{|c|c|c|c|c|c|c|c|c|}
\hline \multirow[t]{2}{*}{ Constructs } & \multirow[t]{2}{*}{ Mean } & \multirow[t]{2}{*}{ sd } & \multirow[t]{2}{*}{ alpha } & \multicolumn{2}{|c|}{ Correlations } & \multirow[b]{2}{*}{3.} & \multirow[b]{2}{*}{4.} & \multirow[b]{2}{*}{5.} \\
\hline & & & & 1. & 2. & & & \\
\hline 1. Risk perception pesticide use & 3.83 & .66 & .94 & & & & & \\
\hline 2. Benefit perception & 2.93 & .59 & .86 & $.39 * *$ & & & & \\
\hline 3. Anxiety & 1.81 & .75 & .80 & -.12 & -.07 & & & \\
\hline 4. Positive emotions & 2.85 & 1.03 & .94 & $.33^{* *}$ & $.63^{* *}$ & -.05 & & \\
\hline 5. Motivation to find information & 3.85 & .71 & .77 & .10 & .11 & -.05 & $.29^{* *}$ & \\
\hline 6. Willingness to pay & .19 & .24 & n.a. & $.30 * *$ & $.43^{* *}$ & -.14 & .43 & -.04 \\
\hline
\end{tabular}

**. Correlation is significant at the 0.01 level (2-tailed); *. Correlation is significant at the 0.05 level (2-tailed).

Note: All constructs were measured on a 5-point Likert scale

Hypotheses testing. Separate ANOVA's were conducted to test for differences between conditions (Table 3.4). There were two significant effects and one marginally significant result. Participants in the positive condition scored marginally higher on the perceived risks of pesticide use compared to the participants in the negative condition, $F(1,85)=2.97, p=.09, \eta^{2}=.03$, which was in line with $\mathrm{H} 1 \mathrm{~b}$. They also scored significantly lower on anxiety (H2b) compared to the participants in the negative condition, $F(1,86)$ $=7.68, p=0.01, \eta^{2}=.08$. In the positive condition, participants were willing to pay 25 cents more for an organic apple compared to a regular apple, whereas this was only 13 cents in the negative condition. The difference between conditions was significant, $F(1$, 80) $=5,48, p=0.02, \eta^{2}=.06$, and confirmed hypothesis $3 \mathrm{~b}$.

There were no significant differences between the conditions for benefit perception (H1a), positive emotions (H2a), and the motivation to find information ( $\mathrm{H} 3 \mathrm{a})$, all $p$ 's > .05 .

Table 3.4 Differences between conditions in means of the constructs -study 2 ( $\mathrm{n}=82-88$ )

\begin{tabular}{llccc}
\hline Constructs & $\begin{array}{c}\text { Positive condition } \\
\text { Mean (std) }\end{array}$ & $\begin{array}{c}\text { Negative condition } \\
\text { Mean (std) }\end{array}$ & F-value & $\begin{array}{c}\text { Sig } \\
\text { 2-tailed }\end{array}$ \\
\hline 1. Risk perception pesticide use & $3.94(.64)$ & $3.70(.67)$ & $\mathbf{2 . 9 7}$ &. $\mathbf{0 9}$ \\
2. Benefit perception & $2.98(.58)$ & $2.88(.60)$ & .56 & .46 \\
3. Anxiety & $1.61(.54)$ & $2.04(.90)$ & $\mathbf{7 . 6 8}$ &. $\mathbf{0 1}$ \\
4. Positive emotions & $2.99(.99)$ & $2.68(1.06)$ & 1.99 & .16 \\
5. Motivation to find information & $3.87(.79)$ & $3.83(.62)$ & .09 & .77 \\
6. Willingness to pay & $.25(.29)$ & $.13(.15)$ & $\mathbf{5 . 4 8}$ & $\mathbf{. 0 2}$ \\
\hline
\end{tabular}




\section{General Discussion}

The Internet is becoming one of the main sources of information. In the Netherlands alone, social media is used by almost $70 \%$ of the Dutch Internet users. Social media enables individuals to share their opinion with a great number of other consumers or organisations. This led us to examine to what extent the online opinions of others on Facebook influences individuals' perceptions, feelings and behaviour. To get insight in the effects of online social proof, in terms of comments and likes on Facebook, two experimental studies were conducted. Organic food was used as the topic of the two experiments, because individuals have to make up their minds regarding purchasing and consuming those products. In this decision process, they might be inclined to follow the lead of similar others.

In the first online experimental study we manipulated both comment valance (positive versus negative) as well as the reinforcement of the comments (the number of likes: high versus low). Though previous research (e.g. Betsch et al., 2011; Kause et al., 2014; Seo et al., 2015; Winterbottom et al., 2008) found evidence that comment valence had an effect on consumer responses, we did not find a main effect of valence on perceptions, emotions or behavioural intentions. One possible reason for this might be that, in particular in relation to organic food, the effect of comments valence is moderated by the evaluation of the comments (Slater \& Rouner, 1996).

There was a strong interaction effect between the valence of the comments and the way in which respondents perceived these comments in terms of clearness and usefulness. It showed that the effect of comment valence was dependent upon how clear and useful respondents perceived the comments to be. When respondents perceived the comments as valuable and clear, explicit social proof had the strongest effect in the expected direction. This is in line with research from Slater and Rouner (1996) that demonstrates the importance of message evaluation as a moderator in a persuasion context. Based on the effect size, this multivariate effect that held for all the dependent variables, can be characterized as small to medium-sized (Hedrick et al., 1993).

In the first study, results also indicated that the interaction between valence and the number of likes was insignificant, meaning that the effect of comment valence was not strengthened by the number of likes. In addition, it was shown that the number of likes did not have an effect on any of the dependent variables. This is in line with what we expected on the basis of other research that showed that the number of likes of a comment did not influence individuals' attitudes (Peter et al., 2014; Winter et al., 2015). It is also consistent with the exemplification theory (Zillmann, 2002), suggesting that individuals are more easily influenced by comments than likes, because comments are perceived as explicit examples or opinions. One might argue that the number of likes becomes more relevant when individuals process information in a heuristic way. 
For example, when under time pressure individuals might not have enough cognitive resources to systematically review all the comments and might therefore depend on the number of likes as a heuristic for validation. Further research is needed to investigate this and examine how information processing styles influence the importance of subtle cues such as likes.

Taken together, the first study showed that online social proof in the form of comment valence can be effective when consumers perceive the comments as valuable and clear. The number of likes, which can be viewed as a more implicit form of online social proof, did not seem to have an additional effect on individuals' attitudes.

The second study was conducted to get insight in the effect of the number of likes when individuals watch both positive and negative comments at the same time as this situation resembles reality more closely (Lee et al., 2008). This second study showed that in such more inconclusive situations subtle cues of social proof, e.g. the number of likes, did have an effect on individuals' reactions (e.g. Jin et al., 2015) regarding organic food.

We found an effect of likes on risk perception of pesticide use (marginal), negative emotions, and willingness to pay. Individuals in the positive condition were willing to pay 25 cent more on average for an organic apple compared to a non-organic apple. This difference was only 13 cents in the negative condition. These results show that subtle cues of social proof can influence perceptions, emotions, and behavioural intentions. It is assumed that individuals need additional information to base their opinion on and start looking at implicit types of social proof, such as likes. This is consistent with research from Amblee and Bui (2011) in relation to online reviews of short e-books: Individuals switched their attention to author ratings when there were no reviews available.

No effects of the number of likes were found regarding benefit perception, positive emotions, and the motivation to find information. One possible explanation for the insignificant effect of our manipulations for some of the dependent variables could be that our research sample already had stable, somewhat positive attitudes towards organic food products. The principle of social proof has been proven to work especially well in ambiguous situations (Cialdini, 2001). This might be less applicable regarding organic food.

Generally, it is predicted that online social proof works especially when individuals are uncertain about a particular topic. The effects of Facebook comments and likes on individuals' attitudes are thus likely to be generalized to other issues of which individuals have to form themselves an opinion and do not seem to be limited to food issues. Further research is needed to provide insight in how individuals are influenced by comments and likes on Facebook about other products than organic foods.

Another interesting question that remains unanswered is how different information sources on Facebook would have impacted individuals' reactions. Study 3.1 shows that 
the way in which the Facebook comments are perceived is an important moderator in the relationship between comment valence and individuals' reactions to the topic. Previous research has demonstrated the importance of source credibility (Slater \& Rouner, 1996), also with respect to individuals' reactions on Facebook (Seo et al., 2015). Individuals might respond differently when information comes from trusted sources, such as friends and family members, compared to unknown others.

As is common in social psychological experimental studies, only participants who correctly filled out the manipulation checks were included in the analyses, resulting in a smaller sample size. Up to $97 \%$ of the participants correctly identified the valence of the comments. However, only about half of the sample (52\%) correctly identified the number of likes. This implies that participants possibly paid more attention to the post and comments, while not focusing on the number of likes. This is in line with the idea that likes are an implicit form of social proof. In study 3.1 the comments provided the participants with sufficient information as the comments were either positive or negative. Therefore the number of likes could have been perceived as irrelevant. Another reason for the high dropout rate in study 3.1 could be that the participant's perceptions of the size of the number of likes did not match our intended manipulation. Specifically, some participants in the conditions with a low number of likes perceived the number of likes to be large. Explorative research about the number of likes showed that participants perceived our low likes manipulation to be a high number of likes, because they use their own Facebook experience as a reference point instead of the reference frame that we provided them with.

In study 2.3, when valence was mixed, one might expect that the subtle cues, the number of likes, should have been more easily noted. This was however not the case as still about $50 \%$ of the sample in study 3.2 failed to correctly answer the manipulation check question. This implies could imply that the participants do no notice the number of likes. Using eye-tracking might be useful to disentangle to what extent individuals do not pay attention to the number of likes, perceive it as irrelevant or are unconscious of these cues.

Together, the two studies conducted shows that social media information, such as comments and likes on Facebook, is influential on individuals' attitudes, feelings and behavioural intentions. These effects are especially present when individuals perceive the explicit social proof as clear and useful, leading to more positive attitudes following positive social proof and more negative attitudes when receiving negative social proof. Organisations that post information online on social media need to be aware that individuals might influence each other by responding to the information. How these comments have an effect on individuals' attitudes is dependent on the clearness of the comments. 
Appendix 3.A: Facebook pictures study 1 in English (translated from Dutch)
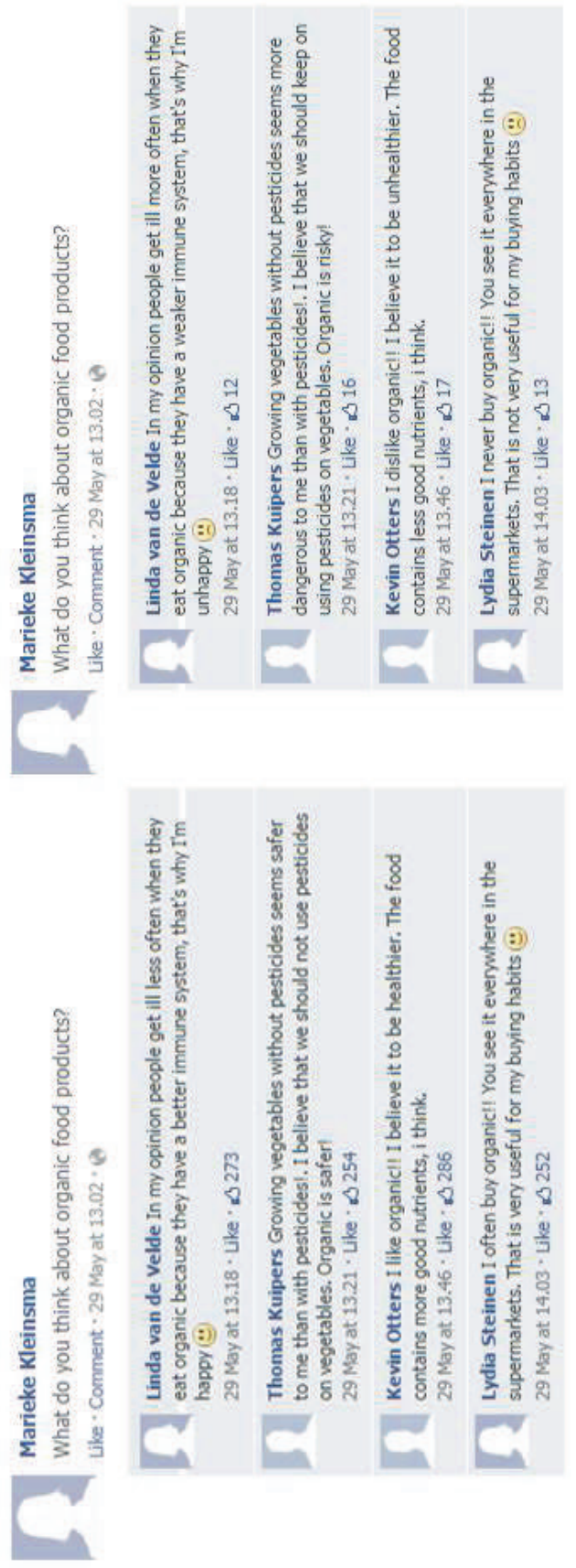


\section{CHAPTER 4}

\section{SOCIAL MEDIA MEDIATED INTERACTION WITH PEERS, EXPERTS AND ANONYMOUS AUTHORS}

Conversation Partner and Message Framing Effects on Risk Perception and Sense-making of Organic Food

This chapter is based on: Hilverda, F., Kuttschreuter, M., \& Giebels, E. (2017). Social Media mediated Interaction with Peers, Experts and Anonymous Authors: Conversation Partner and Message Framing Effects on Risk Perception and Sense-making of Organic Food. Food Quality and Preference, 56, 107-118. 
The Internet is one of the main sources currently used by individuals to search for information about food (Jacob, Mathiasen, \& Powell, 2010; Kuttschreuter et al., 2014; Redmond \& Griffith, 2006; Tian \& Robinson, 2008). When surfing the Internet, individuals may end up on social media sites where they can find the opinions of others; in many cases these are peers or experts. A broad range of research shows that, generally speaking, both the opinions of peers and experts influence the individuals' attitudes and behaviour (Andsager, Bemker, Choi, \& Torwel, 2006; Griskevicius, Cialdini, \& Goldstein, 2008; Pornpitakp, 2004). However, previous research has mainly focused on face-to-face or non-interactive online communication. Furthermore, especially on the Internet, the opinions found are often from anonymous authors. The current importance of online media and the development of social media raise the important question: to what extent does the exchange of opinions during online chats with peers, experts, and anonymous authors influence individuals' risk perception and sense-making and, subsequently, food purchasing decisions?

This experimental study was set up to increase our understanding of the way individuals respond to and make sense of risk and benefit information transmitted via social media. We focused on organic foods, in view of their increasing popularity and availability (Giraud, 2002; Hughner, McDonagh, Prothero, Shultz, \& Stanton, 2007).

The main innovative aspect of this study was the inclusion of an anonymous author as online information source. Social media enables an altered interaction compared to traditional media and face-to-face communication (Dellarocas, 2003), and offers new possibilities for information transfer (Rutsaert et al., 2013a; Veil, Buehner, \& Palenchar, 2011). Interaction via online social media has different characteristics compared to faceto-face communication, including the presence of anonymous authors. On social media, an individual can, for example, more easily be deceived, because users are essentially anonymous and can pretend to be someone other than who they really are (Dellarocas, 2003; Rutsaert et al., 2013a).

This study also adds to the existing literature by examining the effects of providing individuals with risk and benefit information regarding a positively evaluated food topic.

This study, further, provides practical knowledge about the way the social environment influences individuals' processing of food-related information. This knowledge may enable food communicators to adapt their information supply to empower individuals to make well-informed choices. Knowledge of consumer information processing is also very important for food producers, as this knowledge facilitates understanding of consumer preferences and purchasing behaviour. 


\section{Risk perception, information processing and sense-making}

Receiving information about the risks and benefits of particular foods may elicit the need to make sense of and to evaluate these risks and benefits more closely. The active process of seeking, processing and integrating information is labelled "sense-making" (Wilson \& Wilson, 2013). This is the process by which individuals give meaning to the world around them, and sense is its outcome. Sense-making involves the need for information, taking notice of information, seeking information, and integrating new information in such a way that the individual perceives no obvious contradiction between this information and the individual's own original opinions and beliefs (Weick, 1995; Weick, Sutcliffe, \& Obstfeld, 2005; Wilson \& Wilson, 2013). It takes place at both an individual and a collective level (Caughron et al., 2013; Miranda \& Saunders, 2003).

Another means to sense-making is information sharing. Information sharing is related to sense-making in two ways. Firstly, the interaction and exchange of information between the consumer and other individuals or organisations is a means to collective sense-making (Caughron et al., 2013; Miranda \& Saunders, 2003). Secondly, information sharing is a behavioural outcome of sense-making. After sense-making, the individual can decide to share information with others (Yang, Kahlor, \& Griffin, 2013).

\section{Perceptions and sense-making regarding organic foods}

Research shows that individuals generally hold positive attitudes towards eating organic foods, focus on organic food's benefits (Magnusson et al., 2001; Saba \& Messina, 2003), and associate organic food with naturalness (Shafie \& Rennie, 2012). They consider the microbiological risks and those of natural toxins to be small compared to the risks of pesticides (Williams \& Hammitt, 2001), and perceive organic foods to be less risky than conventional foods (Hammitt, 1990). Individuals who are more positive about organic products tend to have less positive attitudes towards pesticide use (DicksonSpillmann, Siegrist, \& Keller, 2011; Saba \& Messina, 2003) as in their perception, there are fewer benefits and more risks attached to the pesticide use (Saba \& Messina, 2003). Such perceptions and attitudes are the main determinant of a preference for organic foods (Aertsens, Verbeke, Mondelaers, \& Van Huylenbroeck, 2009; Hughner et al., 2007; Padel \& Foster, 2005; Saba \& Messina, 2003), however, this preference does not directly translate into actual purchasing behaviour; characteristics like taste and price play a role as well (Lee \& Yun, 2015).

Many studies on food communication and sense-making focus on topics where individuals had ambivalent or negative attitudes, such as red meat (Regan et al., 2014; Rutsaert et al., 2015), or nanotechnology in foods (Frewer et al., 2014; Siegrist, Cousin, Kastenholz, \& Wiek, 2007; Siegrist, Stampfli, Kastenholz, \& Keller, 2008). How risk and benefit information affects the risk perception and sense-making of food products considered to be favourable, is still unclear. 


\section{Message framing}

An online source can frame his/her viewpoint by emphasising specific information. This may have an impact on individuals' reactions. Framing can be defined as the way in which information is presented (Chong \& Druckman, 2007). Emphasis frames (Chong \& Druckman, 2007) are characterised by focusing the attention on certain aspects of a topic (e.g. gains versus losses). Emphasis frames may contain the same information, while putting the focus on different aspects or on different parts of the information.

An important question is whether it makes a difference with respect to individuals' risk perception and sense-making with respect to organic foods if a gains frame (e.g. emphasis on advantages) or a losses frame (e.g. emphasis on disadvantages) is used. These two frames correspond to the distinction between promotion-focus and prevention-focus made in Higgens' Regulatory Focus Theory (Higgens, 1997).

Evidence for a differential effect of frames was reported in a recent study by Yan (2015), who showed that negative health frames induced higher levels of cognitive elaboration with respect to eating junk food compared to positive ones. In real-life situations, however, there often is no clear emphasis on one of the two, and individuals are left uncertain whether the risks outweigh the benefits or vice versa.

We therefore tested whether framing the message of the conversation partner (gains, losses, uncertainty) had an effect on risk perception and sense-making. We hypothesised that:

- $\quad$ The framing of the message of the conversation partner affects risk perception (H1a) and sense-making (H2a). A losses frame is related to higher levels of risk perception and sense-making compared to a gains or uncertainty frame.

\section{Conversation partner, perceived similarity and perceived expertise}

The author of a message and the way this person is perceived in terms of similarity and expertise have been found to influence individuals' information processing behaviour (Paek, Hove, Juong, \& Kim, 2011; Wilson \& Sherrell, 1993). In the context of online interaction on organic food, the differential impact of three conversation partners seems most relevant to study: that of peers, experts and anonymous authors. In the past, individuals often relied on expert information (Lord, 2002). Nowadays, however, individuals mostly use the Internet to find the information they need. They often end up at user-generated webpages (Laurent \& Vickers, 2009) containing information spread by other individuals (Helm, 2000). In an online context, peers are thus becoming increasingly important as information sources. Their contribution is not restricted to factual information, but also includes user experiences which have been shown to affect attitudes and behaviour (Vermeulen \& Seegers, 2009; Winterbottom, Bekker, Conner \& Mooney, 2008; Zhu \& Huberman, 2014). It is yet unclear whether individuals rely more 
on opinions posted online by their peers or still follow professional advice (Dellarocas, 2003). A distinctive feature of the Internet is that the source of the information might be unknown. As a great deal of Internet information has no clear author, a third category of particular interest is that of the anonymous authors.

Peers have been found to be especially influential because individuals are likely to follow the lead of others, when the perceived similarity between the individual and the other is high (Festinger, 1954; Platow et al., 2005). This phenomenon is called social proof (Cialdini, 2001; Griskevicius et al., 2008). The more similar the other person is perceived to be, the more relevant the opinion of this person is for the individual's behaviour, attitudes and beliefs (Festinger, 1954; Pornpitakp, 2004; Salancik \& Pfeffer, 1978). Perceived similarity seems to be a powerful mechanism, as minor shared characteristics are sufficient to create a feeling of similarity (The Minimal Group Paradigm; see Diehl, 1990 for review). Perceived similarity is also associated with attractiveness and a higher level of certainty regarding the opinion of the person (Faraji-rad, Samuelsen, \& Warlop, 2015). A review study examining the impact of social modelling showed that perceived similarity between model figures and individuals is important for consumption and purchasing behaviour (Cruwys, Bevelander, \& Hermans, 2015). Peer feedback has also been found to be influential in the context of social media (Verroen, Gutteling, \& De Vries, 2013).

A second influential feature of an online author is the author's perceived expertise, an important source of authority (Ayeh, 2015; Cialdini \& Goldstein, 2004). This so-called authority principle states that depending on an expert mostly leads to appropriate actions, and that individuals might therefore use experts' opinions and behaviour as a shortcut to decision making (Cialdini, 2001). There is evidence that individuals use the perceived expertise of food communicators as a heuristic to determine the accuracy of a message (Verbeke, 2005).

The mechanisms of perceived similarity and perceived expertise may explain why peers and experts affect consumer responses to information. On social media, it is often uncertain who posted the information, and information about similarity and expertise is also missing. Anonymous authors are considered less credible and the impact of their message is smaller (Rains \& Scott, 2007; Rains, 2007). Thus with regard to organic foods, individuals may be less likely to appreciate the opinions of anonymous authors compared to those of experts or peers. Research suggests that the effect on risk perception also depends on message characteristics (Frewer, Howard, Hedderley, \& Shepherd, 1999).

With respect to sense-making, we expect that individuals experience a feeling of uncertainty when receiving information from an anonymous author (Rains \& Scott, 2007). Because sense-making is especially relevant in uncertain situations (Weick, 
1995; Weick et al., 2005), a higher level of sense-making might be expected when communicating with an anonymous author compared to with a peer or an expert, with the exception of information sharing. A lower level of information sharing and risk perception seems plausible, because individuals might be less convinced of the validity of the information received from an anonymous author. This would mean that the effect of the message frame is also dependent on the conversation partner. Regarding the conversation partner it is predicted that:

- The conversation partner affects risk perception (H1b) and sense-making (H2b). Interacting with a peer or an expert compared to with an anonymous author reduces information need and taking notice of and searching for more information, and increases risk perception and information sharing.

- The effect of the message frame is dependent upon the conversation partner for both risk perception ( $\mathrm{H} 1 \mathrm{c})$ and sense-making $(\mathrm{H} 2 \mathrm{c})$.

\section{Initial attitude}

According to cognitive dissonance theory (Festinger, 1957), individuals are likely to stick to their opinion, which may impact how they search for and process new information. There is evidence that individuals seek information that is in line with their current worldview and avoid information that may cause unpleasant feelings or thoughts (Gaspar et al., 2015; Narayan, Case, \& Edwards, 2011). In an experimental study, Van Dijk, Fischer, De Jonge, Rowe, \& Frewer (2012) found that, following information provision, positive initial attitudes were associated with lower levels of risk perception and higher levels of benefit perception. Initial attitude might also be a proxy for involvement. Research shows that highly involved individuals process information more systematically (Petty, Cacioppo, \& Schumann, 1983). This implies that positive initial attitudes may increase sense-making when compared to less-favourable attitudes. Initial attitudes may thus be an important determinant of individuals' risk perception and sense-making in the context of communicating risk and benefit information on organic foods.

The effect of the message frame expressed in the conversation on risk perception and sense-making may depend on the initial attitudes (Pornpitakp, 2004). Uncertainty frames may strengthen the effect of initial attitudes. Providing information on both the benefits and the risks of eating organic food, without emphasising one or the other, might induce a feeling of uncertainty, as no straightforward conclusion can be drawn. Uncertainty may induce individuals to use their initial attitude as a heuristic to evaluate the information they receive (Kuhn, 2000). When the conversation partner is uncertain about how to weigh the advantages and disadvantages of eating organic food, initial attitudes may thus be an important determinant of risk perception and sense-making. 
We therefore hypothesized that:

- $\quad$ The more positive the initial attitude towards eating organic food, the lower the risk perception (H1d) and the higher the sense-making (H2d).

- $\quad$ The effect of the message frame on risk perception (H1e), and sense-making (H2e) is dependent on the initial attitude towards eating organic food.

\section{Method}

\section{Design and Manipulations}

An experiment was run to investigate to what extent the message frame and the identity of conversation partner influenced risk perception and sense-making of organic food information. We used a 3 (message frame: gains vs losses vs uncertainty) $\times 3$ (conversation partner: peer vs expert vs anonymous) between-participants design. Participants were randomly assigned to one of the nine conditions.

The main part of the experiment consisted of a simulated chat. Participants were told that we were interested in their opinion about eating organic food and that they would discuss the topic beforehand with another participant to help them form an opinion. In reality, there was no conversation partner; participants received preprogrammed messages instead. The first message was aimed at manipulating the conversation partner. In the peer condition, the participants read that their interaction partner was from the same 'blue group' (see procedure), and in the expert condition, that the interaction partner was an expert from the Netherlands Nutrition Centre. In the anonymous condition, no additional information about the interaction partner was given, except that (s) he was participating in the experiment.

The second message contained the message frame. The arguments provided in the message (i.e. the risks and benefits mentioned) were the same across conditions, but the emphasis varied. In the gains condition, the interaction partner was convinced that the benefits outweighed the risks. In the losses condition, the interaction partner was convinced that the risks outweighed the benefits. In the uncertainty condition, the interaction partner had doubts about whether the benefits outweighed the risks or vice versa.

Data collection took place in two waves.

\section{Participants}

Participants were recruited by an internationally well-known market research agency meeting the ICC/ESOMAR International Code on Market and Social Research. This research agency acted in accordance with the ethical standards of the 
institutional research committee. Participants were asked to complete a two-wave online questionnaire which would take them about 30 minutes in total. To ensure representativeness of the Dutch internet users with respect to gender and age, the sample was stratified according to gender and age-groups. Individuals aged under 18 were excluded. In the first wave, the participants' initial attitude towards eating organic food was measured. The second wave formed the main part of the study: the chat.

The research sample of the first wave consisted of a representative sample of the online Dutch population of online media users, $\mathrm{n}=998$. We excluded 7 speeders who completed the questionnaire in less than $1 / 3$ of the median duration. Two days after they completed the first wave, the remaining participants were invited to participate in the second wave.

The second wave was completed by 514 participants. Participants who stopped after the manipulation and continued later $(n=40)$ were excluded from the analysis, as were those whose responses in the chat session indicated that they did not take the investigation seriously $(n=9)$, leaving a sample of 465 .

A manipulation check was conducted ${ }^{5}$. Participants who incorrectly remembered their conversation partner ( $4 \%$ in the peer condition; $31 \%$ in the expert condition, and $21 \%$ in the anonymous condition) or the message frame, were also excluded from further analyses ( $8 \%$ in the gains condition, $21 \%$ in both the losses condition and in the uncertainty condition).

This resulted in a final sample of 310 participants. There were no significant differences in initial attitude, $F(1,989)=2.55, p=.11$, gender, $\chi^{2}=1.75, p=.19$, and agegroup, $\chi^{2}=3.14, p=.08$, between the final sample and drop-outs. Please see Table 4.1 for the distribution of participants per condition, age category and gender.

A randomisation check showed that there were no differences between conditions regarding gender, age, education, online media use, and initial attitude towards. Additionally, the overall evaluation of the conversation did not depend on the message frame, $F(2,305)=.16, p=.85$, nor on the conversation partner, $F(2,305)=1.26, p=.29$.

\footnotetext{
${ }^{5}$ To ensure that the participants understood the manipulations correctly, three questions were asked. To measure the message frame, participants answered the question: "Participant 23 a) thinks that there were more advantages than disadvantages, b) thinks that there were more disadvantages than advantages, or c) doubts whether the advantages outweigh the disadvantages." To measure the conversation partner, participants answered the following two questions: "Participant 23 is... a) a member of the research panel, b) a food expert, c) did not tell me whether he/she was a member of the research panel or a food expert, or d) I don't know anymore" and the question "In which group was participant 23 placed? a) yellow, b) blue, c) red, d) green, e) participant 23 did not tell me in which group he/she was placed, or f) I don't know anymore.
} 
Table 4.1 Number of participants by experimental condition, age and gender

\begin{tabular}{|c|c|c|c|c|c|c|c|c|c|}
\hline \multicolumn{2}{|c|}{ Conversation partner } & \multicolumn{6}{|c|}{ Message frame } & \multicolumn{2}{|c|}{ Total } \\
\hline & & \multicolumn{2}{|c|}{ Gains } & \multicolumn{2}{|c|}{ Uncertainty } & \multicolumn{2}{|c|}{ Losses } & & \\
\hline & & Male & Female & Male & Female & Male & Female & & \\
\hline \multirow[t]{3}{*}{ Expert } & $18-34$ years & 7 & 3 & 2 & 3 & 5 & 9 & 29 & 93 \\
\hline & $35-49$ years & 3 & 7 & 3 & 5 & 7 & 3 & 28 & \\
\hline & 50 year and older & 10 & 5 & 3 & 11 & 3 & 4 & 36 & \\
\hline \multirow[t]{3}{*}{ Peer } & 18 - 34 years & 11 & 10 & 7 & 5 & 5 & 5 & 43 & 118 \\
\hline & $35-49$ years & 4 & 4 & 4 & 5 & 3 & 5 & 25 & \\
\hline & 50 year and older & 3 & 11 & 9 & 10 & 4 & 13 & 50 & \\
\hline \multirow[t]{4}{*}{ Anonymous } & 18 - 34 years & 3 & 8 & 4 & 3 & 6 & 4 & 28 & 99 \\
\hline & 35 - 49 years & 3 & 1 & 6 & 4 & 5 & 4 & 23 & \\
\hline & 50 year and older & 10 & 7 & 7 & 10 & 6 & 8 & 48 & \\
\hline & & 54 & 56 & 45 & 56 & 44 & 55 & & 310 \\
\hline \multicolumn{2}{|l|}{ Total } & \multicolumn{2}{|c|}{110} & \multicolumn{2}{|c|}{101} & \multicolumn{2}{|c|}{99} & & \\
\hline
\end{tabular}

\section{Instruments}

Dependent variables. Risk perception and sense-making (information need, taking notice, searching, sharing) were measured. Scales were mostly adapted from previous research and partly newly developed. Information need and taking notice of information were adapted from Kuttschreuter et al. (2014). With respect to taking notice, participants could indicate that they did not use one of the channels and pick the option "not applicable". The mean score was based on at least three pertinent responses ${ }^{6}$. Items regarding searching for information were adapted from measures developed during the European 'FoodRisC'-project (Barnett et al., 2011). Items for sharing of risk information and risk perception were inspired by other risk related instruments. Items were all measured on a 7-point Likert-scale, except for risk perception, which was measured on a 7-point bipolar scale. Reliability was good. Table 4.2 presents the formulation of the items, the scales and the reliability of the constructs.

Moderators and additional measures. There were three newly developed moderators: initial attitude, perceived similarity, and perceived expertise. Additional measures included reasons to share and search, evaluation of the conversation, certainty of opinion of the partner, and online media use. Items were all measured on a 7-point scale. Reliability was good (Table 4.2).

\footnotetext{
${ }^{6}$ Only one participant picked the "not applicable" option more than three times and was excluded from the analysis.
} 
Table 4.2 Scales, items and reliabilities of constructs $(n=309-310)$

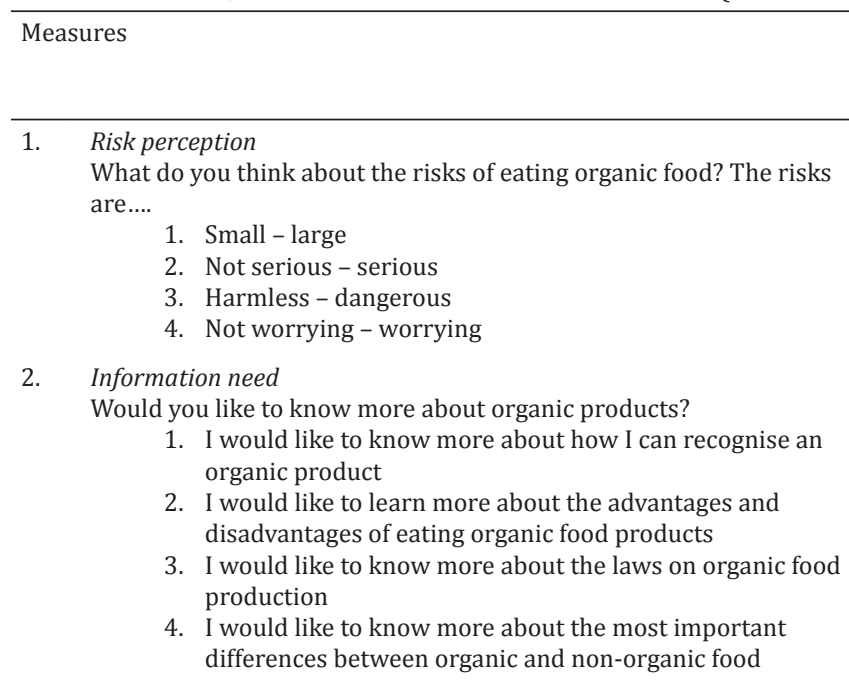

\section{Taking notice of information}

Please indicate how likely you would be to do each of the following things? (If you don't use the media mentioned, choose 'not applicable')

1. Keep an eye out for additional information when watching television

2. Pay attention when I happen to come across a radio broadcast on this topic

3. Keep an eye out for additional information when reading the newspaper

4. Use a search engine like Google to search the internet to find more information about the issue

5. Directly access the internet website of a food communication organisation in my country

6. Visit social media such as Facebook and Twitter to find out more about the issue

4. Searching for information

I'm inclined to search for information about...

1. The disadvantages of organic products

2. The way you prepare organic products to benefit most

3. How to best prepare organic food

4. The way to deal with the possible risks of eating organic food

5. Sharing of risk information

If I encounter an interesting message about eating organic...

1. I'd be inclined to share the information with family and friends

2. I think that I'd talk about organic food in the coming days

3. I'd feel the need to discuss this information with others

4. I'd share the information with individuals who I believe are interested

5. I'd talk about it face-to-face to a friend, relative or acquaintance*

7-point Likert scale from 1 = strongly disagree to

7 = strongly agree

7-point Likert scale from $1=$ very unlikely to 7 = very likely

7-point Likert scale from

7 = strongly agree

7-point Likert scale from 1 = very unlikely to 7 = very likely 
6. Initial attitude

1. What do you think about eating organic products?

7-point bipolar scale

$\mathrm{r}=.79$

Very negative - very positive

2. How important do you believe eating organic food to be? Totally unimportant - very important

7. Perceived similarity

The interaction partner...

1. Is comparable to me

2. Is in the same situation as I am

8. Perceived expertise

The interaction partner...

1. Knows a lot about the topic

2. Is an expert in the field of food

9. Reasons to search and share

If I'm going to search/share information, the reason is ...

1. To confirm the interaction partner's story

2. To check my own ideas

3. To find out what other consumers think about eating organic food

4. To find out more about organic food products

10. Evaluation of the conversation

The conversation was..
1. Easy - difficult
2. Cooperative - competitive
3. Enjoyable - annoying
4. Active - passive
5. Tense - relaxed [recoded]
6. Friendly - hostile
7. Interesting - boring

11. Certainty of opinion of the partner

1. The interaction partner was certain of his/her opinion

$$
\begin{aligned}
& \text { 7-point Likert scale from } \quad \mathrm{r}=.71 \\
& \begin{array}{l}
1=\text { strongly disagree to } \\
7=\text { strongly agree }
\end{array} \\
& \begin{array}{l}
\text { 7-point Likert scale from } \quad \mathrm{r}=.92 \\
1=\text { strongly disagree to } \\
7=\text { strongly agree }
\end{array}
\end{aligned}
$$

7-point Likert scale from 1 = strongly disagree to 7 = strongly agree

Not applicable

7-point bipolar scale .85

12. Online media use

How frequently do you use the following online media?
1. Email
2. Facebook
3. Twitter
4. Skype
5. Forums or blogs

Not applicable Not applicable

\section{Procedure}

First wave. In the first wave, the participants were unaware of the subject of the investigation until opening the link provided in the invitation. They were instructed that, based on their responses to the first wave, a topic for the second wave of the study would be chosen and that they would discuss this topic with another participant. The main purpose of the first wave was to measure the initial attitude towards eating organic food. To conceal this purpose, the participants were asked to evaluate three other food related topics (nanotechnology in foods, genetically modified foods, and food supplements) besides organic food, and they were asked to answer questions about their eating habits and leisure activities. 
Second wave: experiment. The participants received the link to the second part of the study two days after completing the first part. Participants were told that participants in the study included both participants from the research panel as well as employees of the Netherlands Nutrition Centre. A screenshot from the Bionext ${ }^{7}$ website was presented to introduce the subject of organic food.

Participants answered questions giving background information about themselves and their household. They were told that, based on this information, all participants would be placed in groups, with each group given a colour and that participants with similar response patterns would be placed in the same group. All participants were placed in the "blue group". This classification was needed for the similarity manipulation. Though all participants received a message saying that they were placed in the blue group, only the participants in the peer condition were given the information that the conversation partner was in the same group. This means that only participants in the peer condition were manipulated with "similarity" while this was not the case for the other conditions.

After the system allegedly searched for available conversation partners, participants received the first message containing the manipulation of the conversation partner. The conversation partner was subsequently rated on perceived expertise and similarity. The participants then received a second message containing the message frame. They were then asked to send a response with their own opinion. To make the interaction more realistic, elements of an online conversation were added, such as loading icons and typing errors. The texts can be found in Appendix 4.A.

Next, participants evaluated their conversation partner on certainty of opinion, filled out manipulation check questions, and rated the conversation. They then answered questions to measure the dependent variables. Finally, their socio-demographics and online media use were measured. Participants received their reward. During the whole data collection period, participants could contact the helpdesk of the research agency for questions and debriefing.

\section{Analysis}

Analysis of variance was applied to test the hypotheses on risk perception and sense-making (GLM). Assumptions of analyses of variance were tested. The assumption of homogeneity of variance (Levene's test was not significant for any of the dependent variables) was met. In addition, our sample met the assumption that our sample was drawn from a normally distributed population, and that conditions were independent as well as the observations within conditions. The normality assumption was, however,

\footnotetext{
${ }^{7}$ Bionext is concerned with the collective interests in the organic sector in the Netherlands and in Brussels via the IFOAM EU Group. It was founded by farmers, trading bodies and retail associations.
} 
violated. Though visualisation of the data by Q-Qplots and histograms showed that our variables approached a normal distribution, the Shapiro-Wilk test pointed to nonnormality. Based on a review study by Glass, Peckham and Sanders (1972) and a more recent Monte Carlo simulations study by Schmider, Ziegler, Danay, Beyer and Bühner (2010), GLM's were used as their empirical evidence showed that it is robust against violation of normality and no valuable alternatives exist.

\section{Results}

\section{Means}

Risk perception was quite low $(M=2.94)$. Sense-making varied between $M=4.18$ for information need, $M=3.83$ for searching, $M=3.73$ for taking notice, and $M=3.58$ for information sharing. These means make floor and ceiling effects unlikely. Overall, risk perception was negatively related to sense-making. Table 4.3 presents the means, standard deviations, and the correlations among the constructs.

Table 4.3 Means, standard deviation and reliabilities of constructs ( $n=309-310)$

\begin{tabular}{|c|c|c|c|c|c|c|c|c|c|}
\hline \multirow[t]{2}{*}{ Constructs } & \multirow[t]{2}{*}{ Mean } & \multirow[t]{2}{*}{ sd } & \multicolumn{2}{|c|}{ Correlations } & \multirow[b]{2}{*}{3.} & \multirow[b]{2}{*}{4.} & \multirow[b]{2}{*}{5.} & \multirow[b]{2}{*}{6.} & \multirow[b]{2}{*}{7.} \\
\hline & & & 1. & 2. & & & & & \\
\hline 1. Risk perception & 2.94 & 1.35 & & & & & & & \\
\hline 2. Information need & 4.18 & 1.46 & $-.19^{* *}$ & & & & & & \\
\hline 3. Taking notice of information & 3.73 & 1.46 & $-.16^{* *}$ & $.56^{* *}$ & & & & & \\
\hline 4. Searching for information & 3.83 & 1.38 & -.05 & $.63^{* *}$ & $.65^{* *}$ & & & & \\
\hline 5. Sharing of risk information & 3.58 & 1.48 & $-.20^{* *}$ & $.50^{* *}$ & $.75^{* *}$ & $.54^{* *}$ & & & \\
\hline 6. Initial attitude & 4.27 & 1.37 & $-.35^{* *}$ & $.34^{* *}$ & $.38^{* *}$ & $.28^{* *}$ & $.39^{* *}$ & & \\
\hline 7. Perceived similarity & 3.65 & 1.39 & $<-.01$ & $.15^{* *}$ & $.15^{* *}$ & $.14^{*}$ & $.14^{*}$ & .03 & \\
\hline 8. Perceived expertise & 4.15 & 1.60 & -.11 & .02 & .06 & -.02 & .07 & .01 & -.07 \\
\hline
\end{tabular}

**. Correlation is significant at the 0.01 level (2-tailed); *. Correlation is significant at the 0.05 level (2-tailed).

\section{Perceived similarity and expertise of the conversation partner}

Consistent with the manipulation of the conversation partner, there were significant differences between the conditions in both perceived similarity, $F(2,307)=25.22, p$ $<0.001$, and expertise, $F(2,307)=92.89, p<0.001$. Pairwise post-hoc comparisons, using a Bonferroni adjustment, showed that the perceived similarity was rated significantly higher in the peer condition compared to the expert condition, but not compared to the anonymous condition $\left(M_{\text {peer }}=4.00 ; M_{\text {expert }}=2.85 ; M_{\text {anonymous }}=3.98, p<0.001\right)$. Analogously, participants in the expert condition rated the expertise of their partner significantly 
higher compared to participants in the peer $\left(M_{\text {expert }}=5.66 ; M_{\text {peer }}=3.52, p<0.001\right)$ and anonymous condition $\left(M_{\text {anonymous }}=3.51, p<0.001\right)$, while the difference between the peer and anonymous condition was insignificant.

These results suggest that participants in the anonymous condition attributed characteristics to their interaction partner. No information about their interaction partner was provided, yet the evaluation of the partner in terms of perceived similarity and expertise was comparable with evaluations in the peer condition and different compared to the expert condition. This suggests that participants in the anonymous condition perceived their partner as a peer rather than an expert.

The differences found between the conditions in perceived similarity and perceived expertise suggest that these variables could have a moderating role in the relationship between the message frame and risk perception and sense-making.

\section{Perceived certainty of the interaction partner}

The expert $(M=4.67)$ was perceived to be significantly more certain of his/her opinion compared to the peer $(M=3.98, p=.01)$, and marginally more certain compared to the anonymous conversation partner $(M=4.12, p=.05)$, applying the Bonferroni adjustment.

\section{Hypotheses testing}

Analysis of variance was applied. The effects on risk perception were evaluated by performing an GLM. Next, a GLM was conducted predicting information need, taking notice of and searching for additional information as dependent variables. As the hypotheses for information sharing differed from those of the three other elements of sense-making, a separate GLM's was conducted for information sharing.

The model that we tested included main effects for the message frame, conversation partner, initial attitude, perceived similarity, and perceived expertise. Interaction effects of the message frame on the one hand, and the conversation partner, the initial attitude, perceived similarity and perceived expertise ${ }^{8}$ on the other were included in the model to test for moderation effects.

Table 4.4 shows the adjusted means of the constructs when the moderators were kept constant (set equal to the mean, i.e. zero) per condition. Adjusted means were used to avoid the problem that differences between conditions in raw means could be attributable to differences on moderators, rather than real groups differences (Field, 2013).

${ }^{8}$ The attitude towards eating organic food, perceived similarity and perceived expertise were centred around the mean. 


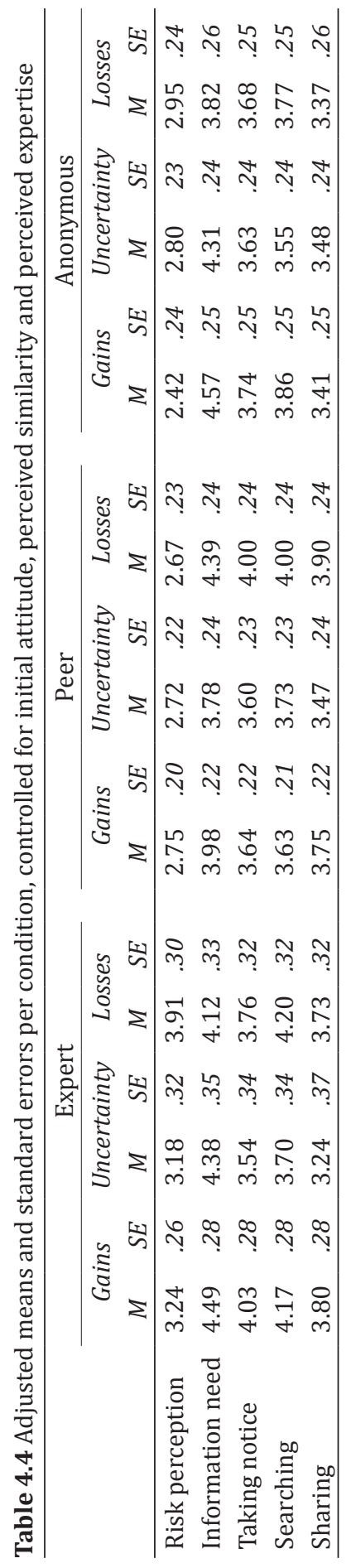


Risk Perception. There was no significant main effect of the message frame on risk perception, $p>.05$, implying that risk perception following the chat was not dependent on how the message was framed. This means that H1a stating that the message frame had an impact on risk perception, was rejected.

There was a significant main effect of conversation partner, $F(2,295)=5.43, p=$ .005 , partial $\eta^{2}=.04$. Post-hoc tests showed that, after all effects had been included in the analysis, risk perception was significantly higher in the expert condition $(M=3.03)$ compared to the peer $(M=2.88)$ and anonymous condition $(M=2.94)$. The difference between the peer and anonymous condition, however, was not significant. H1b was thus partially confirmed.

The interaction between the message frame and the conversation partner was insignificant $p>05$. Hypothesis $1 \mathrm{c}$ was therefore rejected.

\section{Risk Perception and Perceived Expertise by Conversation partner}

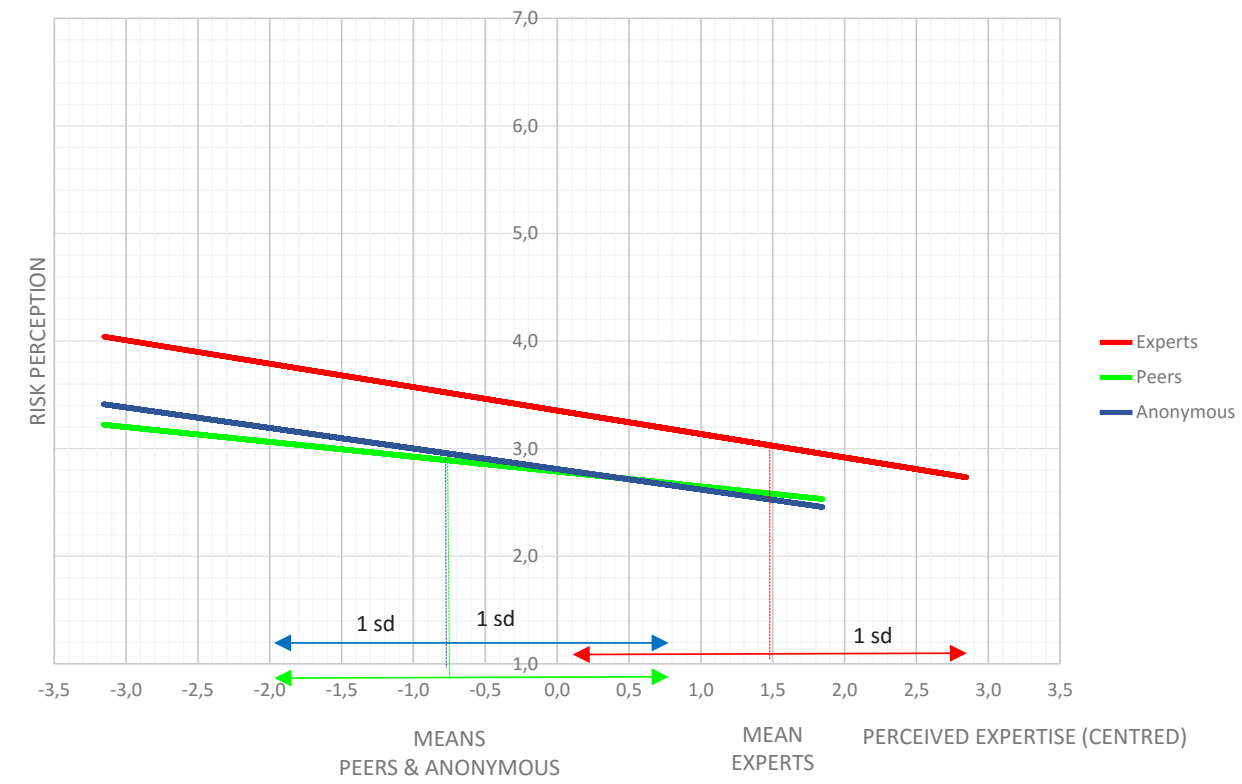

Figure 4.1: Relationship between risk perception and perceived expertise by interaction partner, including means and standard deviation on perceived expertise for the interaction partners

There was a significant main effect of perceived expertise on risk perception, $F(1$, 295) $=13.94, p<.001$, partial $\eta^{2}=.05$. The results showed that, after all effects had been included in the analysis, higher levels of perceived expertise were associated with lower risk perception. There was no significant interaction effect of perceived expertise and the viewpoint of the partner. Figure 4.1 visualises the significant main effects of 
conversation partner and the perceived expertise. This figure also shows the interaction between the conversation partner and perceived expertise.

The main effect of perceived similarity was insignificant, as was the interaction effect of perceived similarity and the message frame.

There was a statistically significant main effect of initial attitude, $F(1,295)=$ 45.12, $p<.001$, partial $\eta^{2}=.13$ : the more positive the initial attitude, the lower the risk perception following the chat. Our results confirmed $\mathrm{H} 1 \mathrm{~d}$. The interaction between the initial attitude and the message frame was insignificant. H1e was therefore rejected.

Sense-making. There were no significant main effects of the message frame on any of the sense-making variables, all $p$ 's $>.05$, implying that sense-making following the chat was not dependent on the frame of the message. H2a stating that the message frame affected sense-making, was therefore rejected.

There were no significant main effects of conversation partner on sense-making, all $p$ 's $>$.05. Hypothesis $2 \mathrm{~b}$ was also rejected.

The interaction between the message frame and the conversation partner was found to be insignificant for all dependent variables, all $p$ 's $>.05$. H2c was therefore rejected.

There was a statistically significant multivariate main effect of perceived similarity on sense-making, $F(3,289)=3.67, p=.01$; Wilk's $\lambda=0.96$, partial $\eta^{2}=.04$. Univariate analysis showed that this effect held for information need, $F(1,291)=8.49, p=.004$, partial $\eta^{2}=.03$, taking notice of information, $F(1,291)=7.28, p=.007$, partial $\eta^{2}=.02$, and searching for additional information, $F(1,291)=7.08, p=.008$, partial $\eta^{2}=.02$. In the separate GLM for information sharing, this main effect was also significant, $F(1,292)$ $=7.11, p=.008$, partial $\eta^{2}=.02$. These results indicate that the higher the perceived similarity of the conversation partner, the more the participants engaged in sensemaking. The interactions between the perceived similarity and the message frame were not significant for any of the sense-making variables.

There was no significant main effect of perceived expertise on sense-making, nor were there significant interaction effects of perceived expertise and the message frame.

There was a statistically significant multivariate main effect of initial attitude, $F$ (3, 289 ) $=18.68, p<.001$; Wilk's $\lambda=0.84$, partial $\eta^{2}=.16$. Subsequent univariate analyses showed that this main effect held for information need, $F(1,291)=37.92, p<.001$, partial $\eta^{2}=.12$, taking notice of information, $F(1,291)=44.39, p<.001$, partial $\eta^{2}=.13$, and searching for additional information, $F(1,291)=24.40, p<.001$, partial $\eta^{2}=.08$. In the separate GLM, a statistically significant main effect of initial attitude on information sharing was also found, $F(1,292)=45.90, p<.001$, partial $\eta^{2}=.14$. Results confirmed $\mathrm{H} 2 \mathrm{~d}$ : the more positive the initial attitude, the more sense-making.

With respect to the interaction effect of the message frame and initial attitude (H2e), there was a statistically significant multivariate effect, $F(6,580)=2.12, p<.05$; Wilk's 
$\lambda=0.96$, partial $\eta^{2}=.02$. Subsequent univariate analyses showed that this effect was significant for information need, $F(2,291)=5.00, p=.007$, partial $\eta^{2}=.03$, marginally significant for taking notice of information, $F(2,291)=2.94, p=.055$, partial $\eta^{2}=.02$, and insignificant for information searching, $F(2,291)=2.13, p=.12$. The interaction means that the effect of initial attitude on information need, and to a lesser extent taking notice of information, was largest when the conversation partner was uncertain. The interaction effect of the message frame and attitude on information sharing was insignificant. H2e was thus partially confirmed.

\section{Additional analyses}

Gender. Additional analyses showed that gender did not affect risk perception, information need, taking notice of information, searching for information, all $p$ 's $>05$. There was a significant effect of gender on information sharing, $F(1,191)=4.66, p=.03$, partial $\eta^{2}=.02$ : women were more inclined to share information than men. Adding gender as a determinant to predict information sharing only changed one of the previously reported results: the interaction between perceived similarity and the message frame was significant, $F(2,291)=3.10, p=.047$, partial $\eta^{2}=.02$. This interaction means that the effect of perceived similarity was most pronounced when the conversation partner was uncertain.

Reasons for searching and sharing. Participants were most inclined to search for information because they wanted to check their own ideas $(M=4.26)$ and to learn more about organic food products $(M=4.24)$. To a lesser extent they would search to get opinions of others $(M=3.73)$, and they were least inclined to search for information to confirm the story of the conversation partner $(M=3.16)$. A similar pattern was found for information sharing, though overall, the intentions were lower: participants were most inclined to share in order to learn more about organic food products $(M=3.97)$, to check their own ideas $(M=3.92)$, and to get opinions of others $(M=3.80)$. They were least inclined to share information to confirm the story of the conversation partner $(M=2.98)$. Motives for seeking and sharing were compared. To check their own ideas, $t(1,309)=5.16, p<.001$, to learn more, $t(1,309)=4.83, p<.001$, and to confirm the story, $t(1,309)=2.67, p=.008$, scored significantly lower regarding sharing compared to searching. There was no difference for 'getting opinions of others'. 


\section{Discussion and conclusion}

There is a rapid growth in and demand for organic food products. As a result, organic food production is (re)emerging (Murdoch \& Miele, 1999). In the Netherlands, in 2014 the organic food market has a total share of $3 \%$ of the food market and the sales increased with more than $10 \%$ (FoodHolland, 2016). This in turn has led to an increase in the provision of new information on the risks attached to these food products. This information may elicit feelings of confusion and anxiety, and a need for more information so that individuals can weigh the pros and cons and make well informed decisions on their food intake (Van Dijk, Fischer \& Frewer, 2011). Individuals may choose to use the Internet to find additional information (Jacob et al., 2010; Kuttschreuter et al., 2014; Redmond \& Griffith, 2006; Tian \& Robinson, 2008) which they then process and make sense of, in order to decide whether to purchase and eat the particular products.

Online social interaction with another person may impact these processes (Chong \& Druckman, 2007; Wilson \& Sherrell, 1993). An important question is whether this also holds if this communication takes place via social media, given their potential importance in food risk communication (Rutsaert et al., 2013a, 2014).

Our experiment investigated whether message frames (gains vs losses vs uncertainty) and conversation partners (expert, peer, anonymous author) affected risk perception and sense-making with respect to organic foods during an online chat. Results showed that the message frame had no effect on risk perception, nor did the interaction between the message frame and the conversation partner. This means $\mathrm{H} 1 \mathrm{a}$ and H1c were rejected. This contrasts with findings by Van Dijk et al. $(2011,2012)$ who found that information frames affected attitudes. A possible explanation for this difference in results is the different use of frames. We used emphasis frames, while Van Dijk et al. $(2011,2012)$ varied the content of the provided information. Emphasis frames are a very subtle manipulation, and as many individuals already hold positive attitudes towards organic food, merely using a promotion or prevention focus message (Higgens, 1997) may have been too weak to affect risk perception. It is, however, also possible that the findings are the result of our chat procedure. While the emphasis of the message was clear and concise, the text disappeared as soon as the participant started typing his/her response. This may have reduced the exposure to the message frame.

What was significant were the identity of the conversation partner and the way in which this partner was perceived. As expected (H1b), results showed main effects for the conversation partner and perceived expertise: when both effects were included in the model, risk perception was higher among those participants who chatted with an expert, and among those who perceived their conversation partner to be of lower expertise. Based on eta squared, both effects can be characterised as small to medium- 
sized (Hedrick, Bickman, \& Rog, 1993). This latter finding is consistent with the literature on trust: the lower the perceived expertise of a source, the lower the trust in that source (Eiser, Stafford, Henneberry, \& Catney, 2009; Garretson, \& Niedrich, 2004), and the lower the trust, the higher the risk perception (Siegrist \& Cvetkovich, 2000; Siegrist, 2000; Viklund, 2003). It is also consistent with finding that individuals perceive anonymous online authors as untrustworthy (Rutsaert, Pieniak, Regan, McConnon, \& Verbeke, 2013b).

The finding that communicating with an expert is related to a higher level of risk perception is consistent with the literature (Ayeh, 2015; Cialdini, 2001; Verbeke, 2005). It is also challenging to risk communicators; it suggests that it is the communicator rather than the message that affects the individuals' risk perception. Perhaps the mere fact that an expert takes the trouble to chat individually signals to individuals that the involved risks are significant. An alternative explanation might be that it is the result of other characteristics than expertise that the participants ascribed to the conversation partner: the experts were perceived to be more certain of their opinion than the peer and the anonymous author. This is consistent with Karmarkar \& Tormala (2010), who found that experts who express certainty induced less positive attitudes towards a restaurant compared to non-experts. This suggests that experts who are certain are more likely to amplify risk perception compared to peers and anonymous authors.

In addition to message and author characteristics, receiver characteristics impacted on consumer responses to food risk information in terms of risk perception. In line with previous findings (Frewer, Howard, \& Shepherd, 1998; Van Dijk et al., 2012) and H1d, results showed that the initial attitude of the receiver affected risk perception following the chat: the more positive individuals initially were about eating organic food, the lower their risk perception. This was a large-sized effect (Hedrick et al., 1993). Unexpectedly ( $\mathrm{H} 1 \mathrm{e})$, the interaction between the message frame and the initial attitude was not significant (Pornpitakp, 2004). There was no evidence that the initial attitudes were more influential when the conversation partner was uncertain.

Results on sense-making showed that the message frame, the identity of the conversation partner, and the interaction of the frame and the partner did not affect sense-making (H2a; H2b; H2c). This contrasts with findings by Yan (2015) who found that information frames affected sense-making. As in the case of risk perception, this can be explained by the fact that we used emphasis frames, while Yan (2015) varied the content of the provided information. The literature suggests that the perception of the conversation partner is important (Andsager et al., 2006; Griskevicius et al., 2008; Pornpitakp, 2004). Our results showed that chatting with a partner who was perceived to be similar increased sense-making which is in line with the literature (Cruwys et al., 2015; Faraji-rad et al., 2015; Platow et al., 2005; Pornpitakp, 2004). This small to 
medium-sized positive effect (Hedrick et al., 1993) held for information need, taking notice of information, searching for information, and information sharing. It is therefore possible that individuals consider information more relevant or more valid if it is provided by an author perceived to be similar to themselves, and they are thus more inclined to make sense of it.

Again (H2d), and in line with previous findings (Frewer et al., 1998; Van Dijk et al., 2012), initial attitude affected sense-making: the more positive individuals initially were about eating organic food, the higher their level of sense-making. This was a large-sized effect (Hedrick et al., 1993). Results also showed that the effects of initial attitudes were more prominent in uncertain situations (H2e). These effects were small to mediumsized (Hedrick et al., 1993). This implied that the participants relied more on their own opinion and initial attitude if the conversation partner was uncertain. If substantiated by further research, these finding will have important implications for organisations planning to be transparent when communicating their uncertainty on a risk.

Our results suggested that participants in the anonymous condition attributed characteristics to their conversation partner comparable to participants in the peer condition. The manipulation in the anonymous condition stated that the conversation partner was participating in the experiment as well. This manipulation might have served as a minimal condition for a group to emerge, and consequently it could be that participants perceived the anonymous author as a peer. It is, however, unclear whether the anonymous author is perceived as peer or the other way around, i.e. the peer is perceived as anonymous.

Our research focused on organically produced foods. As individuals generally hold positive attitudes towards organic foods (Magnusson et al., 2001; Saba \& Messina, 2003), the question arises to what extent our results are applicable to other food products that are viewed less positively, such as foods produced through nanotechnology, and/ or to completely different domains of consumer products. Further research is needed to provide insights into the way online interaction affects risk perception and sensemaking with respect to a less positively evaluated consumer product.

Another interesting question that remains unanswered is how risk perception and sense-making are influenced by viewpoints that differ in content. In our study, we used emphasis frames (gains vs losses vs uncertainty), while the content (organic produce has risks as well as benefits) was identical. Instead of frames, the effects of variations in the content of the message could be studied, such as one-sided (gains or losses) versus two-sided messages (a mixture of gains and losses).

A downside of designs in which prior attitudes are measured is that this measurement could affect the dependent variables. To minimise this, we added a two-day time interval between the two waves of the study. As a result, our sample size halved between the 
first and second wave of the study, which is not uncommon: response rates in online experiments usually vary between $40-70 \%$ (Göritz, 2007). An explanation might be that the participants' experiences in the second wave did not meet their expectations. They were not made aware that the second wave would only focus on organic products. The duration of the second wave was also considerably longer, and the tasks included a simulated chatting session, which called for a higher level of involvement compared to completing Likert-scale questions. The remaining sample was, however, not selective with respect to initial attitude, gender and age, which suggests that the generalizability of the results is not affected.

The artificial chat session could be viewed as a limitation of the study. Participants indicated however that the conversation partner came across as credible and trustworthy. This implies that although the chat session was artificial, it was perceived as realistic.

In line with common practice, participants who incorrectly filled out the manipulation checks were excluded from analyses. This concerned about one third of the participants. This figure is in line with research that shows that up to $46 \%$ of participants fail to follow instructions when participating in experiments (Oppenheimer, Meyvis, \& Davidenko, 2009). It thus remains a challenge for researchers to shape the manipulation in such a way that it motivates the subjects to participate.

Taken together, this study shows that online chat sessions informing and discussing food risks with individuals can be an effective tool to affect risk perception and increase sense-making. Our results show that the effect of such sessions depends in particular on the perception of the conversation partner: chatting with a partner perceived to have a high level of expertise decreases risk perception, while chatting with a partner perceived to be similar increases sense-making. In situations where it is preferred to facilitate informed risk decision making without prompting a high level of risk perception, this may be a challenge, as this requires the risk communicator to be perceived as having a high level of expertise, and at the same time, be perceived as being similar to the audience. Social media might be a valuable communication channel in this respect. Features of these media are useful in both being perceived as a peer, for example by using pictures, as well as being perceived as an expert by adding links to scientific information. Being active on social media might thus be productive in facilitating informed decision making. 
Appendix 4.A: Manipulation text (translated from Dutch)

\section{Conversation partner}

Expert condition:

Hello,

I work as a food consultant and therefore I know a lot about food. Apparently we're going to be discussing organic food products. What do you do, and do you know a lot about food?

Peer condition:

Hello,

I am in the blue group too, as are you. Apparently we're going to be discussing organic food products. What do you do, and do you know a lot about food?

Anonymous condition:

Hello,

I am participant 23 and I am taking part in this study too. Apparently we're going to be discussing organic food products. What do you do, and do you know a lot about food?

\section{Message frame}

Gains:

I think that there are more advantages to organic food than disadvantages. It's surely better for the environment and animals. Ohyes and they're also cultivated without chemical pesticides and fertilizer etc. Even though, there may be more bacteria on vegetables or parasites on animals that make you ill. Still, I believe we shouldn't to worry about this. The advantages are crucial!

What do you think?

Losses:

I think that there are more disadvantages to organic food than advantages. It's supposed to be better for the environment and animals. Oh yes and they're also cultivated without chemical pesticides and fertilizer etc. However, there may be more bacteria on vegetables or parasites on animals that make you ill. Therefore, I believe we should worry about this. The disadvantages are crucial!

What do you think? 
Uncertain:

I think that there are both advantages and disadvantages to organic food. On the one hand it's better for the environment and animals. Oh yes and they're also cultivated without chemical pesticides and fertilizer etc. On the other hand there may be more bacteria on vegetables or parasites on animals that make you ill. I am uncertain whether we should worry about this. There are advantages, but also disadvantages.

What do you think? 


\section{CHAPTER 5}

\section{ONLINE INFORMATION SHARING ABOUT RISKS}

The Case of Organic Food

This chapter is based on: Hilverda, F., \& Kuttschreuter, M. (resubmitted). Online Information Sharing about Risks: The Case of Organic Food. 
Nowadays, there is an abundance of risk information available in a large number of channels and individuals have to make sense of this overload of information. There are three important means to make sense: information seeking, information processing and information sharing. There is ample research into information seeking and processing, and their determinants. Information sharing, however, is less well understood. This holds in particular for the determinants of this behaviour. The popularity of the Internet as a source for food information (Jacob et al., 2010; Kuttschreuter et al., 2014; Redmond \& Griffith, 2006; Tian \& Robinson, 2008) and its interactive features (Mangold \& Faulds, 2009) make it relevant to focus on information sharing in an online context about food issues. In this study, we aim to add to the existing literature by investigating the determinants of online information sharing. We applied structural equation modeling to understand the relationships between the determinants. Organic food products and their risks were chosen as topic for the study. Food is a subject that concerns everyone (Kuttschreuter, 2006). Organically produced foods are becoming more and more common; at the same time one might expect the public to underestimate their risks. To our best knowledge, online information sharing, outside a work team context, about a risk that is likely to be underestimated, is a novel topic.

Most research on information sharing has been conducted in relation to the sharing of information in teams or workgroups in order to study its impact on group performance. A meta-analysis (Mesmer-Magnus \& DeChurch, 2009) showed a strong effect of information sharing on performance. Task demonstrability, well-structured and cooperative discussion, overlapping information distribution, being informationally independent and membership similarity were positively linked to information sharing. A review article (Berger, 2014) on offline information sharing outside the work environment (i.e. interpersonal communication), showed that individuals share information depending on the situation. For example, they share information about food most often when they are in a restaurant. Five main motives to share information were extracted: impression management, emotion regulation, information acquisition, social bonding, and persuasion.

Little attention has been given to what motivates individuals to share information online in a broader societal context. The available research on the motives to share information via social media mostly focused on the characteristics and gratifications of the media channel (e.g. Oh \& Syn, 2015), and it did not specify the topic and content of the information that was to be shared. An exception is a study by Yang et al. (2013) who used the Risk Information Seeking and Processing model (RISP-model) that has been extensively used to understand information seeking and processing behaviour (see the meta-analysis by Yang, Aloe, \& Feeley, 2014), to examine information sharing regarding climate change. It was found that social norm and information seeking activities were 
most strongly related to information sharing. In the food domain the RISP-model has been tested on eating Great Lakes fish (Griffin, Neuwirth, Giese \& Dunwoody, 2002; Griffin et al., 2004) examining the risks of eating poisoned fish and potential parasitic infection from drinking tap water. These studies, however, focused on information insufficiency and information processing behaviour, not on information sharing or seeking.

The popularity of the Internet and social media made it relevant to focus on online information sharing. The Internet has become one of the main sources of food information (Jacob et al., 2010; Kuttschreuter et al., 2014; Redmond et al., 2006; Tian \& Robinson, 2008) and social media provide individuals with an easy-to-use tool to communicate their ideas. They can now respond to information that is available online and post messages, pictures and video's publicly themselves. With the emergence of social media, the one-way information flow, from communicator to consumer, thus changed into a new and dynamic environment that enabled individuals to post, spread and exchange information rapidly with thousands of other individuals (Mangold \& Faulds, 2009). Research has shown that individuals value food risk information coming from social sources, such as family and friends (Kornelis, De Jonge, Frewer, \& Dagevos, 2007).

New food products, such as organic foods, are currently entering the market on an almost daily basis. Individuals have to decide whether or not to buy these products. Previous research showed that individuals evaluate organic agriculture as quite positive (Magnusson, Arvola, Koivisto Hursti, Åberg, \& Sjödén, 2001; Saba \& Messina, 2003). They perceive organic food to be of higher quality (Kahl et al., 2012; Pearson, Henryks, \& Jones, 2011; Saba \& Messina, 2003), to taste better (Grankvist \& Biel, 2001; Hay, 1989; Roitner-Schobesberger, Darnhofer, Somsook, \& Vogl, 2008; Schifferstein \& Ophuis, 1998), to be healthier (Hay, 1989; Petrescu et al., 2013; Roitner-Schobesberger, et al., 2008), and more nutritious compared to conventional food products (Hay, 1989) and to be better for the environment (Grankvist \& Biel, 2007; Roitner-Schobesberger, et al., 2008) and animal welfare. With respect to Dutch individuals, research showed that organic products were associated with animal welfare, price, health, pesticides, and naturalness. Organic vegetables were linked to human health, while organic meat was associated with animal welfare (Hilverda, Jurgens, \& Kuttschreuter, 2016). Dutch authorities are positive about organic production techniques and are funding new initiatives in this respect (www.bionext.nl). Overall, the attitude regarding organic products in the Netherlands is positive. Newspapers have, however, also reported on scientific research that casts doubts on the claims that organic products are more healthy and better for the environment. 
There are, however, possible risks attached to organic production (Magkos, Arvaniti, \& Zampelas, 2006). Whereas, for example, pesticides are used in traditional agriculture to reduce the risk of bacterial contamination, organic agriculture is characterized by the absence of the pesticides use, which might lead to an increased risk of microbiological contamination (Hammitt, 1990). Scientific evidence of the relationship between eating organic food and an increased risk of microbiological contamination is inconclusive (Bourn \& Prescott, 2002). Some researchers (Stephenson, 1997) believe that organic food increases the risk of microbiological contamination and thus food poisoning, while others (Schmidt, 1999) believe that this risk is comparable to the risk of conventional foods. To enable individuals to make well-informed decisions on the purchase and consumption of organic foods, risk communicators thus need to inform individuals of the possible risks and benefits involved. It should be made clear that organic does not automatically mean safe (Magkos et al., 2006).

In this study we examined online information sharing about the risks of organic food products. We aimed to gain insight in the characteristics of this behaviour and in the main determinants that motivate individuals to share information about these food risks online. We applied structural equation modelling (SEM) to understand the process underlying information sharing.

The results of this study are very relevant to risk communicators. Knowledge about consumer online information sharing behaviour and its instigators will enable them to enhance online sharing behaviour, thereby facilitating well-informed decision making regarding food choice among the general public. This consumer information sharing behaviour might be useful to risk communicators to make individuals aware of chronic risks attached to certain food products, as well as to quickly spread the word during times of food crises.

\section{Information sharing}

The current society can be characterized by a need for "simplexity": complexity of thought combined with simplicity of action (Colville, Brown, \& Pye, 2012). A central aspect of such a society is the desire to make sense of complex information. Sensemaking is the process by which individuals give meaning to the world around them, and sense is the outcome of this process. Sense-making involves recognizing a problem, seeking, finding and integrating new information in such a way that there is no substantial contradiction between the newly encountered information and one's own opinion and beliefs (Weick, 1995; Weick, Sutcliffe, \& Obstfeld, 2005). 
Information sharing is an important means to sense-making, as are information seeking and processing. Sharing (one-way communication) or exchanging information (two-way interaction) can be seen as a way in which the interaction among individuals enhances sense-making (Caughron et al., 2013) and the social construction of meaning (Miranda \& Saunders, 2003).Sense-making does not only involve one's own observations, but the observations of other individuals as well (Dervin, 1998). Information sharing is an observable behavioural aspect of information processing (Yang et al., 2013).

\section{Determinants of information sharing}

We defined four categories of determinants: social determinants, individuals' beliefs about sharing, information-related determinants, and risk perception related determinants. These determinants are all predicted to affect information sharing, directly or indirectly.

\section{Social determinants}

Food choices are oftentimes the result of a social process. The individual who is responsible for preparing the meals often takes the decisions about the foods in consultation with the other household members. In addition, perceptions of risk are socially constructed within a certain culture (Dake, 1992). This makes it essential to take social influences into account when investigating the determinants of information sharing behaviour. We identified three potentially relevant social predictors: interest within the social environment in organic produce, social norm, and sociability.

Being part of a group plays an essential role in human behaviour (Tajfel \& Turner, 1979). The way individuals think, feel, and behave is influenced by the actual, imagined or implied presence of other persons (Fiske, 2009). Identification with a community positively relates to sharing knowledge in a group (Chiu, Hsu, \& Wang, 2006; Hsu \& Lin, 2008). In our study, the social environment is defined as socially close others, such as friends and family. We hypothesize that the higher the interest within the individual's social environment regarding organic foods the higher the intention to share information. This relationship is predicted to be indirect via information insufficiency. Information insufficiency is the core of the RISP-model and can be described as the gap between perceived knowledge and an information sufficiency threshold. If individuals believe that they do not have enough information, they will be motivated to gain information, and hence start to search for it (Griffin, Dunwoody, \& Neuwirth, 1999; Griffin et al., 2008; Kahlor, 2010). 
The Theory of Planned Behavior as well as the RISP-model, which was based on the principles of the Theory of Planned Behavior, emphasize the importance of social norm on behaviour. Social norms reflect the perceived expectations in the social environment. Several studies found that social norm was directly related to information sharing behaviour (Chiu et al., 2006; Lin, Featherman, \& Sarker, 2013; Yang et al., 2013). Hsu and Lin (2008), however, did not find such an effect on knowledge sharing in blogs.

We further hypothesized sociability to be an important construct in relation to information sharing. Sociability is a sub-dimension of extraversion. Individuals who score high on this trait, enjoy social interactions and feel positive about talking about their daily lives with others (De Vries, Ashton \& Lee, 2009). We therefore expected that these individuals would also feel more pressure from their social environment (i.e. social norm) compared to individuals who score low on sociability.

\section{Determinants related to the individual's beliefs about sharing}

The second category of determinants we defined, are determinants related to the individual's beliefs about sharing information.

The most important determinant related to the individual's believes about sharing information is personal outcome expectancies. The expected consequences of sharing information were divided into three major categories: physical effects, social effects and self-evaluation effects (Bandura, 1997; Hsu, Ju, Yen, \& Chang, 2007). Most research showed personal outcome expectancies to predict online information sharing directly (He \& Wei, 2009; Hsu \& Lin, 2008; Hsu et al., 2007; Lin et al., 2013; Roberts, Hann, \& Slaughter, 2006), while the results of some studies pointed to indirect effects via attitude towards sharing (Bock \& Kim, 2002; Papadopoulos, Stamati, \& Nopparuch, 2013). Attitude towards sharing can be viewed as the result of the outcome expectancies, i.e. after evaluating the expected outcomes the consumer has formed an opinion about the usefulness of information sharing, and has been found to predict information sharing (Bock \& Kim, 2002; Hsu \& Lin, 2008; Lin et al., 2013; Papadopoulos et al., 2013).

Self-efficacy is another possible determinants. This is one of the central constructs in the Theory of Planned Behavior. Its importance is also emphasized in the RISP-model. In relation to online information sharing, self-efficacy can be understood in terms of the perceived capability to share knowledge by using the Internet. Based on research by Hsu et al. (2007), Kankanhalli et al. (2005), and Papadopoulos et al. (2013) who all found that self-efficacy beliefs predicted knowledge sharing in virtual communities, a positive relationship between self-efficacy and information sharing is hypothesized (Bock \& Kim, 2002). 


\section{Information-related determinants}

Information-related mechanisms, such as the relevance of being informed and information dependency, were predicted to be important indirect determinants of information sharing. To the best of our knowledge, these determinants have been largely neglected in research on information sharing.

For some individuals, it might be more relevant to be informed about food issues than for others. Ter Huurne and Gutteling (2008) found that participants with higher levels of involvement and personal relevance, were less satisfied with their level of knowledge on the topic and had a higher score on information insufficiency. Based on these results, we predicted that the higher the individual's perceived level of relevance to be informed, the higher the feeling of information insufficiency.

We also hypothesized that information dependency would predict the individual's level of information (in)sufficiency. In line with results by Kuttschreuter et al. (2014), we assume that individuals with a larger general motivation to be well-informed, would experience a higher level of information insufficiency.

A positive relationship between information insufficiency and the intention to share information was further expected. It was hypothesized that individuals who experience a high level of information insufficiency would be more inclined to seek and share information.

The final information-related determinant was information seeking. Yang et al.(2013) found that active information seeking was one of the most important determinants in predicting information sharing regarding climate change. It was therefore predicted that information seeking was directly related to information sharing and that individuals who were likely to search for information would also be likely to share this information with others.

\section{Risk perception related determinants}

On the basis of the RISP-model, we included three determinants that relate to the perceived risks of organic food produce: trust, risk perception and anxiety.

Trust has been found to be a determinant of information seeking and processing. Results showed that the higher the individual's level of trust in retail, trust in the competence of authorities and commercial enterprises, the lower the individual's level of risk perception (Siegrist, 2000; Siegrist \& Cvetkovich, 2000; Viklund, 2003). It is therefore hypothesized that there is a negative correlation between trust and risk perception: the more trust individuals have, the less risks they perceive.

Risk perception has been found to be evoke feelings of anxiety in general, as well as in relation to food issues (Griffin, Neuwirth, Dunwoody, \& Giese, 2004; Kahlor, 2010; Kuttschreuter, 2006), which in turn influenced information seeking (Huurne \& Gutteling, 
2008; Kuttschreuter, 2006). One could argue that risk perception may directly affect information sharing. When individuals have a high level of risk perception they may feel the need to share risk information with others. In addition, this process may also go via anxiety. That is, higher risk perception is associated with higher levels of anxiety, which in turn may lead individuals to share information with others. We therefore hypothesized that risk perception affected information sharing behaviour both directly and indirect, through anxiety.

Anxiety was also included in our model of information sharing. Yang et al. (2013) showed that negative affect was positively related to information sharing: the more negative feelings individuals experienced towards climate change, the more inclined they were to share information about this topic with others. We detailed negative affect by focusing on the anxiety related to the risks of eating organic foods.

\section{Material and methods}

\section{Participants and Procedure}

Respondents were recruited during November 2014 and January 2015 by an internationally well-known, ISO 26362-certificated research agency that conducted the research according to ethical standards. The sample was stratified to be representative for the Dutch population of online media users with respect to age and gender. As a total of $97 \%$ of the Dutch population uses the Internet (CBS), age (18-34 years: $26.5 \%$; 35-49 years: $29.1 \%$; $50+$ years: $44.4 \%$ ) and gender distribution of the Dutch population (male: $49.5 \%$; female: $50.5 \%$ ) were used as a reference points. A soft launch of the survey in which about $10 \%$ of the target group were approached, was ascertain the quality of the questionnaire. The remaining $90 \%$ of the sample was then approached. Participants were requested to fill out an online questionnaire, which took about 20 minutes to complete. They were informed about the purpose of the study.

The research sample consisted of 535 respondents who were aged between 18-87 years old living in the Netherlands (mean age $=48.9$ ). Representativeness was evaluated by comparing the age and gender distribution with the set target distributions. Distribution across age categories was as follows: a total of 127 participants were aged between 18-34 years old (23.7\%), 140 participants were aged between 35 - 49 years old (26.2\%), and 268 participants were 50 years old or above (50.1\%). This distribution differed slightly from the Dutch population, $\chi^{2}(2)=7.03, p=.03$, in a way that the older individuals were overrepresented. With respect to gender, a total of 271 males (50.7\%) and 264 females (49.3\%) participated in this study. These percentages did not significantly differ from the Dutch population, $\chi^{2}=.29, p=.59$. There was a broad range 
in educational level and household composition, and the participants lived across the country in areas of various degrees of urbanization.

A total of $79 \%$ of the participants indicated that they were mainly responsible for doing the grocery shopping in the last month, while $95 \%$ indicated that they were at least once a month responsible for grocery shopping. A total of $90 \%$ of the participants were responsible for cooking dinner at least once in the last week, with $31 \%$ being responsible for this every day. The largest part of the participants (34\%) bought sometimes organic food, while only $2 \%$ bought organic almost always. Of the remaining participants $14 \%$ bought organic food often, 14\% rarely, 21\% almost never and 14\% never bought organic food.

\section{Measurement}

Outcome variable: Online information sharing. The extent to which individuals were inclined to share information about the risks of organic food online was measured by 8 items $(\alpha=.97)$. Respondents indicated on a 7-point scale how likely it was that they would share an interesting message stating eating organic food has risks online. The items related to two modes of online information sharing: sharing publicly (4 items), and privately ( 4 items).

We also measured with whom (5 items, 7-point scale) and through which communication channel (5 items, 7-point scale) the participants were inclined to share information on organic produce. Please see Appendix 5.A for all items.

Social determinants. Respondents were requested to indicate on a 7-point scale to what extent they agreed with four statements on the interest within their social environment with regard to the risks of organic products, for example "My friends are interested in information about the risks of organic products" $(\alpha=.98)$. These items were newly created for this study.

Social norm of sharing was measured by four items on a 7-point scale. Respondents were asked to indicate to what extent they perceived their social environment to expect them to share information about the risks of organic food $(\alpha=.98$; adapted from Yang et al., 2013).

The HEXACO-subdimension sociability was measured by four items (7-point scale; $\alpha=.90$; based on De Vries, Ashton \& Lee, 2009). Items were adapted to the current purpose. Sociability measures the tendency to enjoy social interaction and talk to others.

Determinants related to individual's beliefs about sharing. Outcome expectancies was conceptualized to consist of four components: reciprocity ( 3 items, $\alpha=.94$ ), social effects ( 3 items, $\alpha=.94$ ), self-evaluation effects ( 3 items, $\alpha=.97$ ), and general attitude towards sharing ( 4 items, $\alpha=$.97). All items were measured on a 7-point scale. They were based on scales developed by Chui et al. (2006) and Hsu et al. $(2007,2008)$. The reliability of the total scale was good $(\alpha=.96)$. 
Self-efficacy was measured by four statements regarding the respondent's perceived capability to successfully share information through the Internet (7-point scale; $\alpha=.96$; based on Kuttschreuter et al., 2014).

Information-related determinants. Respondents filled out to what extent they agreed with four statements about the relevance of being well informed about food issues given their personal situation (7-point scale; $\alpha=.95$ ). Items were adapted from measures developed within the European 'FoodRisC' project; Barnett et al., 2011).

Information dependency was measured by four statements regarding the importance of being well informed in one's daily life in general (7-point scale; $\alpha=.91$; based on Kuttschreuter et al., 2014).

Information insufficiency was measured by eight items measuring to what extent the respondents were interested in information about organic food and to what extent they would like to know more about organic food (7-point scale; $\alpha=.97$; based on Kuttschreuter et al., 2014). By assessing individuals' interest in information and information needs we measure information insufficiency in a direct manner ${ }^{9}$, following the approach by ter Huurne (2008) and Eagly and Chaiken (1993).

Information seeking was measured by four items regarding the participants' inclination to search for information about organic food and the risks involved herein (7-point scale; $\alpha=.94$ ). Items were based on Hilverda, Kuttschreuter \& Giebels, 2017.

Risk-perception related determinants. Risk perception was measured by four statements regarding the negative consequences of eating organic food (7-point scale; $\alpha$ $=.94)$. Participants indicated for instance to what extent they considered eating organic food to be detrimental to their health. Items were based on Hilverda, Kuttschreuter \& Giebels (2017).

We measured anxiety by asking respondents to what extent they experienced four emotional states when thinking about the risks of eating organic food (7-point scale, $\alpha=.98$ ). The items (anxious, concerned, afraid and worried) were adapted from Kuttschreuter (2006) and Yang et al. (2013).

Four types of trust were distinguished. Respondents filled out to what extent they agreed that organic products sold in supermarkets were safe to eat (trust in retail; 3 items, 7-point scale, $\alpha=.93$ ) and to what extent they agreed with statements about the organisations that play a role in the safe keeping of the food supply. These were

\footnotetext{
${ }^{9}$ Our measure is a direct measure of information insufficiency that fits the conceptual idea of information sufficiency from the RISP-model. Other studies that measured information insufficiency often used a difference score between the perceived knowledge and the information sufficiency threshold of consumers. This type of measurement implies that consumers make decisions in a very conscious cognitive way which might not always be the case. Consumers might be aware of the gap between the magnitude of the information that they would ideally need (information sufficiency threshold) and their current knowledge, while this might not bother them nor impact their information seeking and sharing behavior.
} 
the Netherlands Food and Consumer Product Safety Authority (4 items, 7-point scale, $\alpha=.95$ ), the main consumer organisation in the Netherlands ('De Consumentenbond', 7 -point scale, 4 items, $\alpha=.93$ ), and food producing companies (4 items, 7-point scale, $\alpha$ $=.92$ ). Items were adapted from measures developed within the European 'FoodRisC' project; Barnett et al., 2011).

\section{Analysis}

Correlations between the determinants and information sharing were composed to examine their relationship with information sharing. Given the large number of determinants and the associated complexity, we reduced our conceptual model to its core and examined the relationship between the most proximal determinants and information sharing in the structural equation model. This provides us with information about the principal determinants regarding online information sharing of organic foods. Structural equation modeling was applied to test this model. An initial model was composed based on a pilot-study on a student sample and subsequently tested by means of AMOS 19 on a sample that was representative of the Dutch population of online media users. We used two-step modelling (Kline, 2005): we first tested the measurement model (CFA), followed by a full structural model that included both the measurement and the structural model.

\section{Results}

\section{Information sharing}

Results showed that online information sharing on average was low $(M=2.77, S D=$ 1.48) and that participants were more inclined to share information privately $(M=2.96$; $S D=1.54)$ than publicly $(M=2.59 ; S D=1.51)$.

In addition, it was examined with whom and how individuals would share risk information with others. Information sharing depended on the person with whom the information was shared (see Table 5.1). Participants were most inclined to share information with a specific person $(M=3.83, S D=1.90)$ or a family member $(M=3.79$, $S D=1.85)$. The mean score for sharing information with friends $(M=3.41, S D=1.83)$ and colleagues $(M=3.25, S D=1.77)$ were both below the mid-point of the scale. The participants indicated to be least inclined to share information publicly $(M=2.77, S D=$ 1.72).

Table 5.1 also shows the type of communication channel respondents would be inclined to use to share information about the risks of organic foods. They would be most inclined to share information in a conversation $(M=4.17, S D=1.87)$ or by email 
$(M=3.46, S D=1.95)$. Social media were less popular: all social media scores fell below the midpoint of the scale. Facebook $(M=3.12, S D=2.01)$ scored quite high, compared to online chat $(M=2.21, S D=1.59)$, forum or blog $(M=2.10 ; S D=1.50)$ and Twitter $(M$ $=2.02, S D=1.50$ ).

Table 5.1 Means and standard deviation of with whom and how respondents would share information about the risk of eating organic $(n=535)$

\begin{tabular}{|c|c|c|c|c|c|c|}
\hline \multicolumn{2}{|c|}{ With whom? } & \multirow{2}{*}{$\begin{array}{c}\text { Mean } \\
3.83\end{array}$} & \multirow{2}{*}{$\frac{\mathrm{sd}}{1.90}$} & \multirow{2}{*}{$\begin{array}{l}\text { How? } \\
\text { 1. In a conversation }\end{array}$} & \multirow{2}{*}{$\begin{array}{c}\text { Mean } \\
4.17\end{array}$} & \multirow{2}{*}{$\frac{\mathrm{sd}}{1.87}$} \\
\hline 1. & Specific person & & & & & \\
\hline 2. & Family & 3.79 & 1.85 & 2. By email & 3.46 & 1.95 \\
\hline 3. & Friends & 3.41 & 1.83 & 3. On Facebook & 3.12 & 2.01 \\
\hline 4. & Colleagues & 3.25 & 1.77 & 4. Via chat (e.g. Skype) & 2.21 & 1.59 \\
\hline \multirow[t]{2}{*}{5.} & Public & 2.77 & 1.72 & 5. Forum or blog & 2.10 & 1.50 \\
\hline & & & & 6. On Twitter & 2.02 & 1.50 \\
\hline
\end{tabular}

Note. 7-point Likert scales, ranging from 1 to 7

\section{Information Sharing and its Determinants}

Table 5.2 shows the means, standard deviations and Pearson correlations of the determinants and online information sharing. Whereas, overall, the participants liked to engage with others $(M=4.78, S D=1.14)$, their social environment did not seem to expect them to share food risk information $(M=2.74, S D=1.44)$ and was only somewhat interested in information about organic food $(M=3.55, S D=1.41)$. Expected outcomes were slightly below the middle of the scale $(M=3.60, S D=1.24)$. Reciprocity outcome expectations dominated $(M=3.94, S D=1.34)$, followed by general attitude towards sharing $(M=3.64, S D=1.58)$, self-evaluation outcomes $(M=3.53, S D=1.43)$ and social outcomes $(M=3.26, S D=1.32)$. This means that the participants were most inclined to share information online with others because they anticipated to receive information in return, to a lesser extent because they perceived information sharing to be useful and to feel good about themselves (e.g. satisfaction), and the least to gain respect or maintain social relationships. The participants felt quite able to share information online $(M=$ $5.02, S D=1.50$ ).

Information dependency was high $(M=4.77, S D=1.15)$. Information insufficiency $(M=4.20, S D=1.39)$ was in the middle of the scale, as was the relevance of being well informed regarding food issues $(M=4.14, S D=1.37)$. Information seeking was slightly below the middle of the scale $(M=3.73, S D=3.73)$. Risk perception and anxiety were low ( $M=2.53, S D=1.20$ and $M=2.03, S D=1.29$ respectively), while trust was above the midpoint of the scale $(M=4.42, S D=1.13)$. 


\section{Relationship between Information Sharing and Determinants}

There were strong correlations between information sharing and the social determinants, the determinants related to individuals' beliefs about sharing, and the information-related determinants.

With regard to the social determinants, the interest within the social environment $(r=.52)$ and social norm $(r=.64)$ correlated strongly positively with information sharing. This means that the more interested and involved the social environment was perceived to be in the topic of organic food, and the more the individual felt that it was expected to share information about the risk of eating organic foods, the more likely the participants were to share information with others. Sociability was less important than interest within the social environment and social norm, but still correlated significantly with information sharing $(r=.24)$.

Information sharing correlated strongly with outcome expectancies of sharing $(r=$ .65): the higher the expected outcomes the more inclined (s)he was to share information online. Self-efficacy correlated weak $(r=.10)$ with information sharing.

Of the information-related determinants, information insufficiency $(r=.46)$ and information seeking $(r=.56)$ showed the strongest correlations with information sharing. This means that a higher level of information insufficiency and a higher intention to search for information corresponded to higher levels of the intention to share information online. Relevance $(r=.39)$ and information dependency $(r=.25)$ correlated positively with information sharing. This means that the more relevant individuals thought it was to be informed and the higher their general information need was, the more likely they were to share information.

The correlations between information sharing and the risk-related determinants were weak. Of these, anxiety showed the strongest correlation with information sharing $(r=.18)$.

Please see Table 5.2 for the correlations between the determinants.

\section{Model testing}

The most proximal determinants were included in the model that was examined through structural equation modelling. These determinants form the core of the model. The following determinants were included in the model: interest within the social environment, social norm, sociability, outcome expectancies, information insufficiency, information seeking, risk perception and anxiety. Please see Figure 5.1 for the model and the results of its evaluation. 


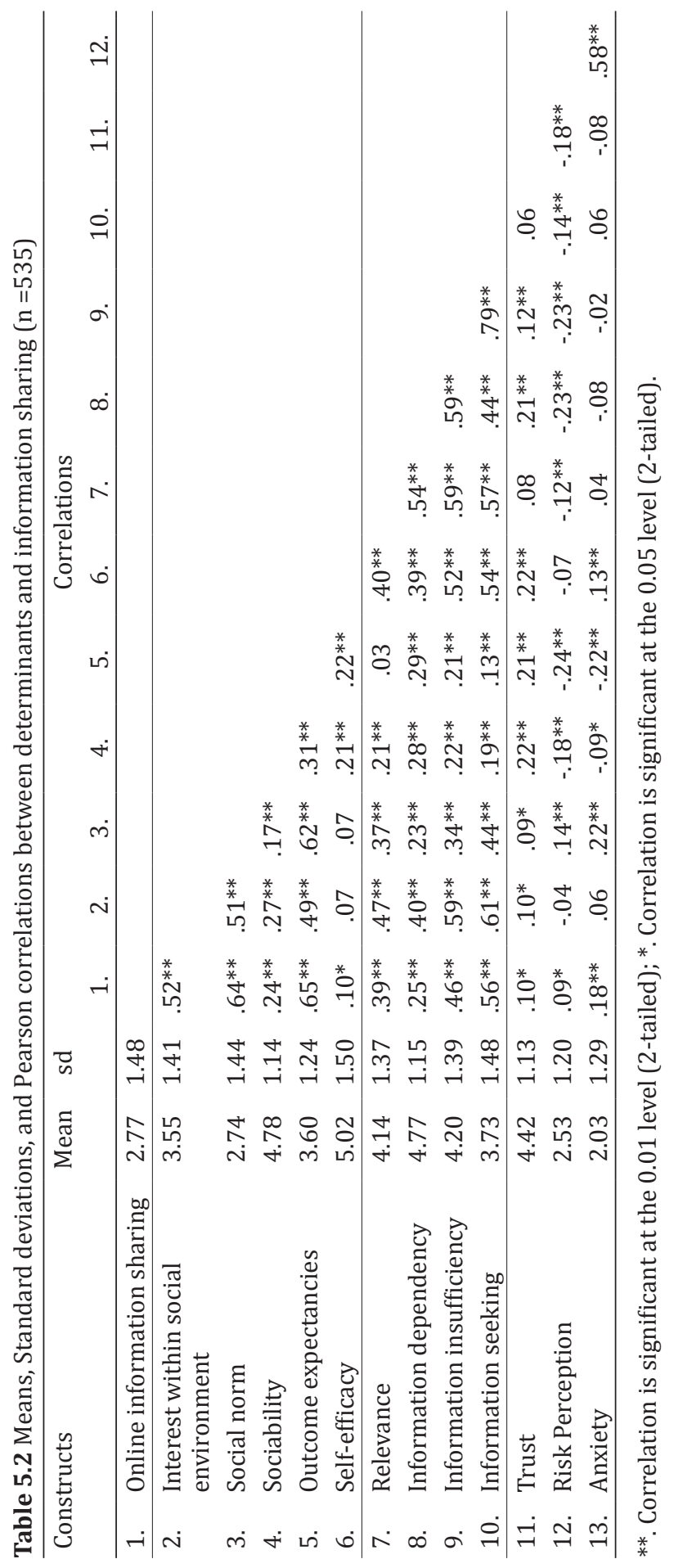




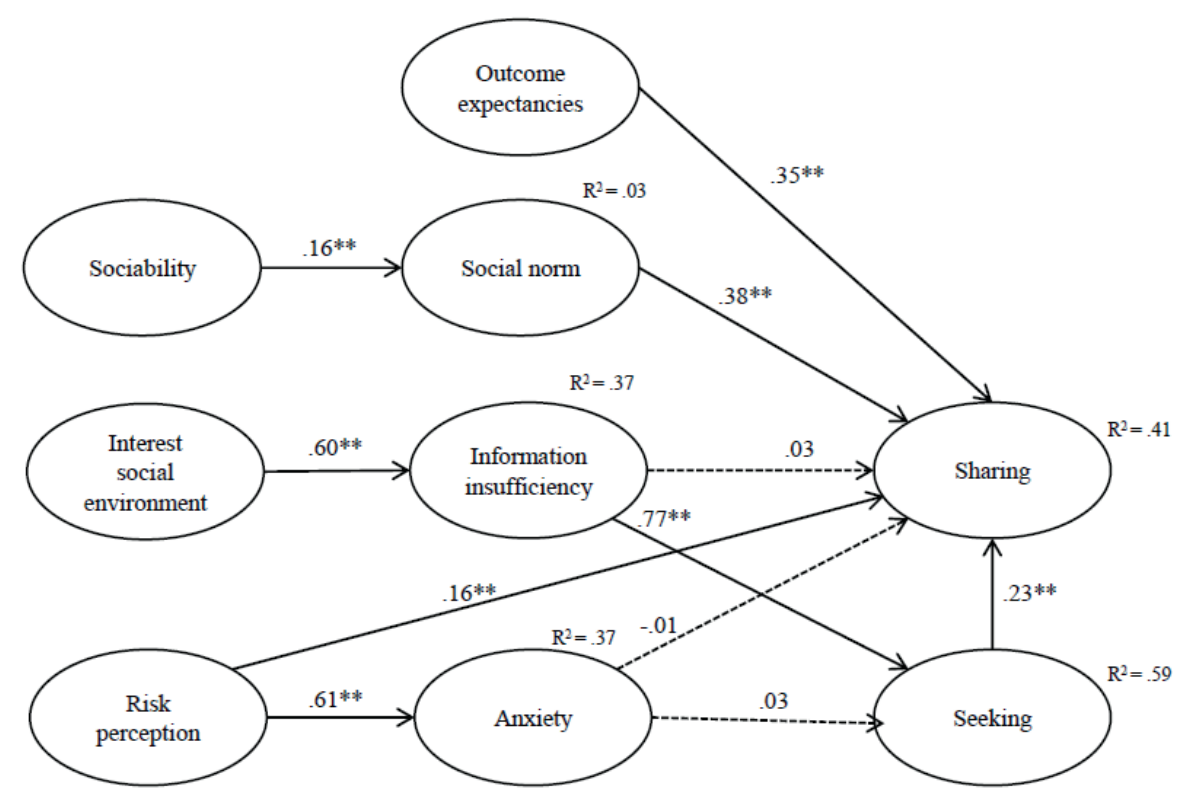

Figure 5.1: Model of Online information sharing, including standardized path coefficients and squared multiple correlations

**. Standardised coefficient is significant at the 0.01 level (2-tailed); *. Standardised coefficient is significant at the 0.05 level (2-tailed)

The measurement model. Generally speaking, the underlying constructs were measured by a set of single item indicators. The exception was outcome-expectancy where four composite indicators were used. The measurement model was tested and convergent validity and discriminant validity were evaluated.

Meeting the most commonly used criteria (Raykov, Tomer, \& Nesselroade, 1991), the measurement model proved to have a good fit ${ }^{10}$ (see Table 5.3 for the observed fit indices and criteria applied to assess model fit). The Root Mean Square Error of Approximation (RMSEA) was 0.07, indicating a good fit (Kline, 2005), as did the normed chi-square of 3.63 (Bollen, 1989; Kline, 2005). The Comparative-Fit Index (CFI) was 0.93, Tucker-Lewis Index (TLI) was 0.92 and the Normed-Fit Index (NFI) was 0.91; all larger than the 0.90 Marcoulides and Schumacker (2013) and Bollen (1989) proposed

\footnotetext{
${ }^{10}$ To improve model fit, for risk perception, information sharing and information seeking the error terms of two indicators were allowed to covary. These relaxations of the measurement model error made sense conceptually. The relevant items with respect to risk perception both related to the risks of organic produce at a general level, whereas the other items focused more specifically to health risks. The relevant items with respect to information seeking both focused on disadvantages of organic produce, whereas the remaining items focused on preparing organic food in a way that one benefits the most. The respective information sharing items asked about sharing risk information with friends and with people the individual knew well; groups that are obviously connected.
} 
as a cut-off point for a good fit. There were two fit indices indicating a poor fit. The chi-square statistic, $\chi^{2}(863)=3129$, was significant and the value of the goodness-offit index (GFI), a transformation of the chi-square, of 0.77 fell below the acceptability threshold. This was to be expected as the chi-square test (and consequently the GFI) are highly dependent on sample size and model size (Fornell \& Larcker, 1981). Considering our relatively large sample size, these measures of goodness-of-fit were less applicable to our study and the obtained values did thus not provide evidence for a poor model fit.

Convergent validity, assessed on the basis of CFA-factor loadings, reliability and average variances extracted (Hair, Black, Babin, Anderson, \& Tatham, 2006), was good. Factor loadings ranged between 0.70 and 0.98 , and exceeded the satisfactory threshold of 0.70 (Chin, 1998). Cronbach's alpha's and the composite CFA-reliabilities were all above the cut-off point of 0.70 . The average variances extracted (AVE) in the CFA were between 0.681 and 0.93 , exceeding the acceptability value of 0.50 (Fornell \& Larcker, 1981). Please see Table 5.4 for factor loadings, composite reliabilities, and average variance extracted.

Table 5.3 Model fit of the measurement model and the structural model ( $\mathrm{n}=535)$

\begin{tabular}{llll}
\hline & Thresholds for acceptable fit & Measurement model & Structural model \\
\hline$\chi^{2}(d f)$ & - & $3129(863)$ & $3635(882)$ \\
$\chi^{2} /$ df & $<3.00-5.00$ & 3.63 & 4.12 \\
RMSEA & $<.05-.08$ & .07 & .076 \\
GFI & $>.90$ & .77 & .75 \\
CFI & $>.90$ & .93 & .92 \\
TLI & $>.90$ & .92 & .91 \\
NFI & $>.90$ & .91 & .89 \\
\hline
\end{tabular}

Table 5.4 Factor loadings, composite reliability estimates and average variance extracted (n $=535$ )

\begin{tabular}{llccc}
\hline Constructs & $\begin{array}{c}\text { Standardized } \\
\text { factor loadings }\end{array}$ & $\begin{array}{c}\text { Composite } \\
\text { reliability }\end{array}$ & $\begin{array}{c}\text { Variance } \\
\text { extracted }\end{array}$ \\
\hline 1. & Online information sharing (8 items) & $.72-.97$ & .97 & .80 \\
2. & Interest within social environment (4 items) & $.92-.97$ & .98 & .91 \\
3. & Social norm (4 items) & $.93-.98$ & .98 & .91 \\
4. & Sociability (4 items) & $.70-.93$ & .90 & .70 \\
5. & Outcome expectancies (4 subscales) & $.71-.94$ & .89 & .68 \\
6. & Information insufficiency (8 items) & $.81-.94$ & .97 & .79 \\
7. $\quad$ Information seeking (4 items) & $.75-.97$ & .95 & .81 \\
8. & Risk Perception (4 items) & $.82-.97$ & .94 & .78 \\
9. & Anxiety (4 items) & $.95-.98$ & .98 & .93 \\
\hline
\end{tabular}


Discriminant validity was assessed by comparing the Pearson correlations between all the constructs computed in SPSS (cf. Table 5.2) with the square root of the AVE's obtained in AMOS (cf. Table 5.4). All square roots of AVE were found to be larger than the inter-construct correlations, pointing to satisfactory discriminant validity (Chin, 1998). Given that our constructs were highly reliably measured, high levels of multicollinearity were tolerable (Grewal, Cote, \& Baumgartner, 2004). Multicollinearity was nevertheless assessed by studying the correlations between the constructs. Correlations varied between -0.02 and 0.79 , and of all 78 correlations only one ${ }^{11}$ approached the threshold for multicollinearity of .80 suggested by Tabachnick and Fidell (2001) and the threshold of 0.85 proposed by Hair et al. (2006). The discriminant validity was therefore considered to be fine.

Based on the above mentioned statistics, it was concluded that the constructs were measured in a reliable way and that the measurement model met the requirements for fitting the full structural model.

The structural model. The full model, visualized in Figure 5.1, was tested. The fit of the model was first evaluated. RMSEA was 0.076, indicating a good fit. CFI was 0.92 and TLI 0.91 , both exceeding the threshold of 0.90 . The NFI was 0.89 , closely reaching the threshold of 0.90 . The normed chi-square of 4.12 also pointed to a good model fit. The chi-square statistic, $\chi^{2}(882)=3635$, was significant, however, and GFI was 0.75 , which was to be expected as we found the same results in testing the measurement model. The fit of the full model was therefore considered to be good ${ }^{12}$.

The model explained $41 \%$ of the variance in the outcome variable information sharing (see Figure 5.1). With respect to the social determinants, 3\% of the variance in social norm was explained. With respect to the information-related determinants, the model explained $37 \%$ of the variance in information insufficiency and $59 \%$ of the variance in information seeking was explained. The model further explained $37 \%$ of the variance in anxiety.

Table 5.5 shows the standardized indirect, direct and total effects of the determinants on information sharing, while Table 5.6 shows the covariance between the exogenous predictors. With respect to the social determinants, social norm proved to have the strongest total effect on information sharing (total effect $=.38$ ): a large direct effect (direct effect $=.38$ ). Sociability had an indirect effect (indirect effect $=.06$ ) on sharing via social norm. The interest within the social environment had an indirect effect (indirect effect $=.12$ ) through information insufficiency.

\footnotetext{
${ }^{11}$ This was the correlation between information insufficiency and seeking information about organic food.

${ }^{12}$ Alternative models were tested, but did not produce a better model fit.
} 
Looking at the determinants related to individuals' beliefs about sharing, it is shown that outcome expectancies has a strong direct effect (direct effect $=.35$ ) on information sharing. This effect is comparable to the effect of social norm on information sharing.

Information-related determinants also had an effect on information sharing. Information seeking had the largest effect (direct effect $=.23$ ) on information sharing. There was no significant direct effect of information insufficiency (direct effect $=.03$ ). This determinant affected information sharing via information seeking (indirect effect $=.17)$.

With respect to the risk perception related determinants, there was a mediumsized direct effect of risk perception (direct effect $=.16$ ) on information sharing, while the other paths were insignificant. This implies that the risk-perception related determinants were less important in predicting information sharing compared to the other categories of determinants.

With respect to the covariances between exogenous predictors (Table 5.6) it is noteworthy to mention the high covariance between interest within the social environment and outcome expectancies (covariance $=.64$ ). This implies that interest within the social environment regarding organic food and the outcomes expected from sharing information are closely related.

Table 5.5 Standardized indirect, direct and total effects of predictors on information sharing (n $=535$ )

Indirect effects Direct effects Total effects

\begin{tabular}{lccc}
\hline Interest within social environment & .12 & - & .12 \\
Social norm & - & .38 & .38 \\
Sociability & .06 & - & .06 \\
Outcome expectancies & - & .35 & .35 \\
Information insufficiency & .17 & .03 & .21 \\
Information seeking & - & .23 & .23 \\
Risk Perception & -.001 & .16 & .16 \\
Anxiety & .006 & -.008 & -.002 \\
\hline
\end{tabular}


Table 5.6 Covariances between exogenous predictors $(n=535)$

\begin{tabular}{|c|c|c|c|c|c|}
\hline & \multicolumn{4}{|c|}{ Covariances } \\
\hline \multicolumn{2}{|c|}{ Constructs } & \multirow{2}{*}{$\frac{1 .}{1.00}$} & \multirow[t]{2}{*}{2.} & \multirow[t]{2}{*}{3.} & \multirow[t]{2}{*}{4.} \\
\hline 1. & Outcome expectancies & & & & \\
\hline 2. & Sociability & $.31^{* *}$ & 1.00 & & \\
\hline 3. & Interest within social environment & $.64^{* *}$ & $.37^{* *}$ & 1.00 & \\
\hline 4. & Risk perception & -.01 & $-.22^{* *}$ & -.08 & 1.00 \\
\hline
\end{tabular}

\section{Discussion and conclusion}

\section{Discussion of empirical results}

Sense-making is an important concept in current society. That is, individuals have to make sense of the abundance of information available in a large number of channels to form an opinion and decide whether or not to buy a product. Information sharing, information seeking and information processing are three important means to sensemaking (Caughron et al., 2013; Miranda \& Saunders, 2003; Yang et al., 2013). Though there is a lot of research conducted about information seeking and processing (Yang et al., 2014), (online) information sharing has been given little attention.

To fill this gap, this study aimed to gain insight in the characteristics of information sharing and the main determinants that motivate individuals to share risk information. The study focused on the risks of organic products, which is motivated by the fact that Dutch individuals perceive organic foods positively, while they seem to be unaware of the possible risks involved.

Based on the Theory of Planned Behavior (Ajzen, 1991) and the RISP model (Griffin, et al., 1999), this study was the first to examine a model predicting online information sharing in relation to food risks. The model included social determinants, determinants related to individuals' beliefs about information sharing, informationrelated determinants and determinants related to risk perception. Structural equation modeling was used to test a model on a representative sample of Dutch Internet users. Constructs were reliably measured.

Results showed that sharing information on the risks of organic produce was low. Respondents were most inclined to share such information with a specific person in a conversation. Online information sharing and information sharing via social media were less popular. Facebook proved to be the most popular social media channel to share information.

There were strong correlations between the relevance of being well informed and information dependency, and both constructs correlated strongly and positively with 
information insufficiency and information seeking. This implies that the more relevant a topic is perceived to be and the more information an individual generally needs, the higher their feelings of information insufficiency and their intention to seek information.

The SEM analyses showed that both the CFA-model and the structural model had a good fit. The model explained $41 \%$ of the variance in information sharing. This is comparable to the approximately $50 \%$ of the variance in information sharing about climate change reported by Yang et al. (2013). Results further showed that the social norm and outcome expectancies were most important in predicting information sharing on food risks. Social norm, e.g. the individuals' perception of the expectations within their social environment, had the strongest direct influence on information sharing. This is in line with results found by Lin et al. (2013), Chiu et al. (2006), and Yang et al. (2013), who showed that social norm was an important predictor of sharing information. This stands in contrast to results reported by Hsu and Lin (2008) who did not find an effect of social norm on information sharing in blogs (on a diversity of topics). This can perhaps be attributed to the fact that Hsu and Lin explicitly focused on blog-users, whereas the participants in our study would not easily engage in blogging.

Outcome expectancies had an important role in predicting online information sharing. This is in line with research from (Hsu \& Lin, 2008; Lin et al., 2013; He \&Wei, 2009; Hsu et al., 2007; Roberts et al., 2006). Information seeking had the third strongest direct effect on information sharing. This effect was somewhat less important in predicting online information sharing than social norms and outcomes expectancies.

The fourth direct path, from risk perception to information sharing, was much weaker compared to the other paths. The fact that risk perception and anxiety turned out to be less relevant might, perhaps, be explained by the fact our study focused on a topic that individuals associate with benefits rather than risks (Avitia Rodríguez, Gil Roig, \& Costa Font, 2011; Hilverda, Jurgens, \& Kuttschreuter, 2016; Hilverda, Kuttschreuter, \& Giebels, 2017). This might also imply that individuals perceived this risk as an impersonal risk, i.e. risks that are a direct threat to something other than the self, instead of a personal risk. Previous research (Kahlor, Dunwoody, Griffin \& Neuwirth, 2006) showed that social norms were most important in predicting information seeking and processing in relation to impersonal risks, while other relations were less prominent. This might explain our low effect of risk perception and anxiety and the strong effect of social norms.

In the structural model, there was a predicted path from information insufficiency to information sharing. This path was, however, insignificant. This means that the effect of information insufficiency was indirect, through information seeking.

The exact relationship between information seeking and sharing is not yet clear, however. The model hypothesizing a causal path from seeking to sharing fitted just 
as well as one hypothesizing a path from sharing to seeking and one hypothesizing common error variance. In line with the ideas of Veinot (2009) the fitted model seems most plausible: the higher the involvement within the social environment regarding organic foods, the more (joint) information seeking and the more information sharing. Further research might help to understand these relationships in detail.

An important subsequent question was what relationships the model did not explain and how these relationships can be understood. The modification indices showed in particular substantive residual correlations between social norm on the one hand, and outcome expectancies and interest within the social environment on the other. This implies that the social pressure of the environment is related to the expected outcomes that individuals perceive and is closely related to the interest within the social environment. There is a social aspect in outcome expectancy that may explain the residual correlation between social norm and outcome expectancies. The same reasoning goes for interest within the social environment: the social aspect of the interest within the social environment is reflected in outcome expectancy and social norms.

All in all, the Theory of Planned Behavior and the RISP model thus proved to be very helpful in understanding information sharing behaviour with respect to the risks of organic produce. This is a very interesting result. In the past, the RISP model was mainly used to explain information behaviour in relation to topics with a negatively connotation, such as external safety (Huurne \& Gutteling, 2008), influenza (Clarke \& McComas, 2012), and climate change (Yang et al., 2013). Our research adds to this research and showed that the RISP-model was also useful in understanding information sharing regarding the risks of organic produce which is perceived as very beneficial and of low-risk (Hilverda et al., 2016; Hilverda et al., 2017), resulting in hardly any anxiety, among Dutch individuals.

\section{Future research}

There are still a number of questions for further investigation. First of all, further research is needed to examine why individuals want to share risk information. In our research we focused on reciprocity, and social and self-evaluation outcome expectancies as potential benefits of information sharing. Results suggested that reciprocity was the main motive to share information, which is in line with research by Liou, Chih, Hsu, and Huang (2015). Research by Baker and Moor (2008) suggested, however, that individuals share information online to express emotions and gain social support. It would be worthwhile to investigate to what extent emotional expression is a relevant motive to share information in a food risk related context. 
Motives to share information online might also depend on the specific social media channel. Oh and Syn (2015) showed that Facebook users were more motivated by enjoyment, altruism, social engagement and reciprocity compared to Twitter users, implying that different motives are prevalent for users of different social media channels. Some channels might thus be used more often to share information in the hope to receive information in return, while other channels might be used more frequently to maintain relationships or to express emotions. Further research is needed to examine how motives to share information differ among particular social media channels.

Another focus for future research would be to examine the relationship between information (in)sufficiency and information seeking and sharing more closely. Empirical support for the role of information insufficiency varies: in some studies (e.g. the present study and (Huurne \& Gutteling, 2008) it was found to be a significant predictor of information seeking, whereas in others it was not (Clarke \& McComas, 2012), or not convincingly (Yang, et al., 2014). The manner in which information insufficiency was measured, varied as well. A systematic analysis of the predictive power of the various operationalisations of information insufficiency is indicated.

An interesting question is, of course, to what extent the model is generalizable to other food products, and to other risks. Our model related to organic foods that are characterized by a low level of perceived risks and anxiety. For other foods with a more negative image a different model might be more explanatory. Risk perception and anxiety might then have a larger effect on information sharing. The question is also whether our model would also hold for another category of risks, for example for those to which individuals are involuntarily exposed. Future research is indicated to investigate to what extent our model is applicable in other contexts and whether the estimated coefficients are of similar size as the ones found in the present study.

\section{Implications for risk communication}

Social media have substantively changed the way individuals interact with each other and with organisations. Individuals can now share their views and experiences publicly and compare them with those shared by others who are similar to them. This might stimulate them to make sense out of all the acquired information.

This consumer information sharing and exchanging behaviour might be very useful to food risk communicators. Nowadays, food risk communication often aims at enhancing informed decision making regarding food choice. As public online information exchange stimulates consumer sense-making, it also enhances informed decision making. Stimulating information sharing might thus be useful as it helps individuals in making well-informed decisions. To stimulate information sharing, risk communicators should be appreciate that the social environment plays an important role in online 
information sharing and make use of this information by, for example, targeting groups of individuals instead of merely focusing on the individual. Risk communicators should also explore the potentials of communication with consumer groups, e.g. the consumer in his/her social environment, in addition to approaching individuals on an individual basis. Social media might be a useful tool for this purpose.

An option to facilitate consumer information exchange would be to allow individuals to react to information posted on one's website. Another option would be to be active on social media one self, respond to consumer comments, and try to start conversations (Veil et al., 2011), e.g. when one would like to increase consumer awareness of risks attached to particular food products. Such a strategy might prove to be productive as well as efficient as it corresponds to the current way individuals are interacting with each other. 


\section{Appendix 5.A Scales, items and reliabilities of constructs ( $n=535)$}

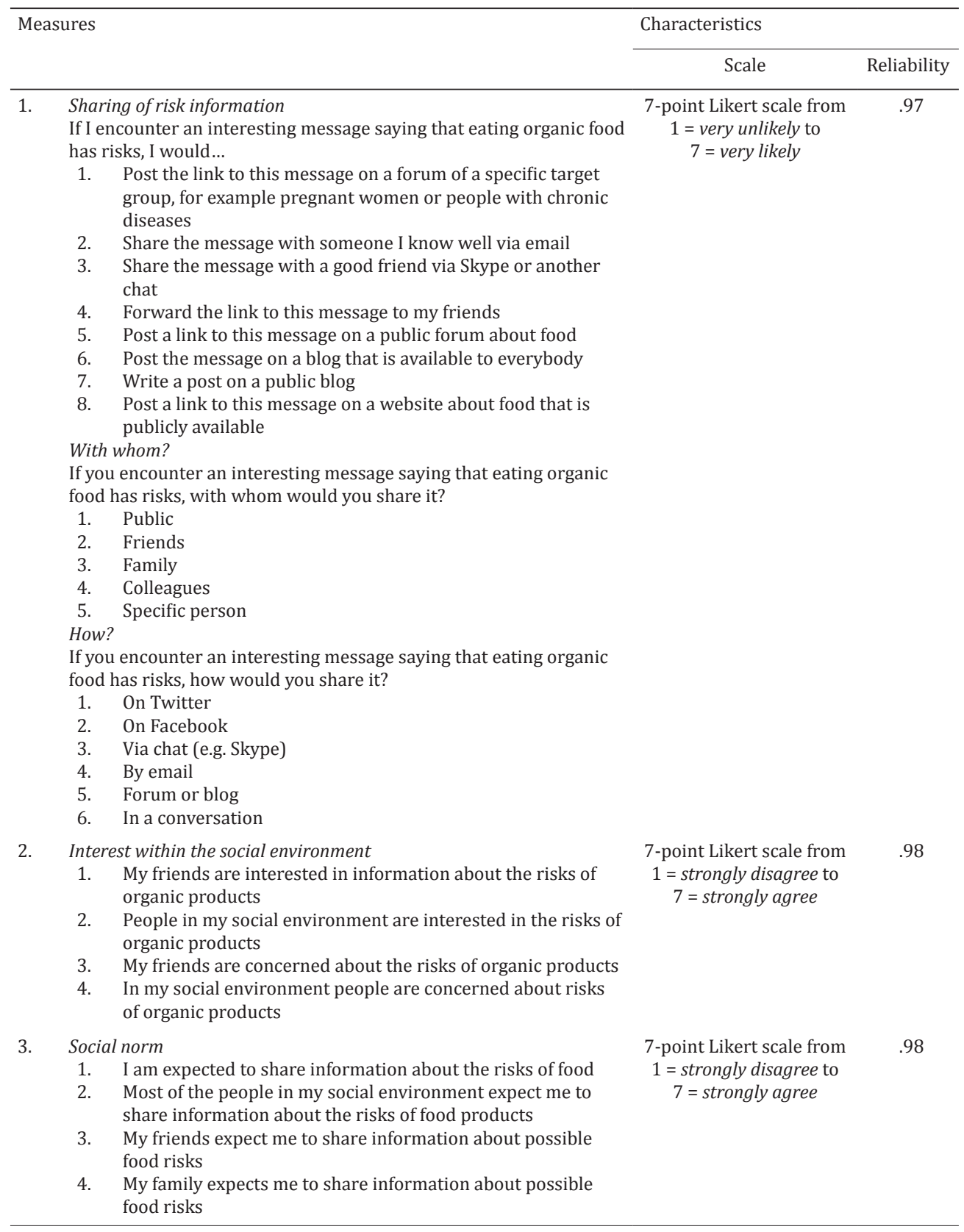


4. Sociability

1. I like to tell to my friends what I've done

2. I enjoy having a lot of people around to talk to

3. I like to talk about what I've experienced

4. I enjoy talking with others about what I've experienced

5. Self-efficacy

1. I am capable to share information via online media with others

2. I know what to do to share a message publicly on the Internet

3. I am capable to safely share information online with my friends

4. It takes me little effort to share a message online

6. Outcome expectancies

Reciprocity:

When I share information about food risks...

1. I'll receive information in return

2. Other people will tell me what they know about these risks too

3. I expect that other people share such information with me in the future

Social:

When I share information about food risks...

4. I'll gain respect

5. This is beneficial for my relationship with family members

6. This has positive consequences for my reputation

Self:

When I share information about food risks...

7. This makes me feel good

8. I'll feel satisfied

9. I'll feel that I'm doing something important

General Attitude towards sharing:

If I encounter an interesting message about food risks...

10. I think it's useful to share the information

11. I think it's wise to share the information

12. I think it's helpful to share the information

13. I think positive about sharing the information

7. Relevance

1. In my personal situation it is very important to be informed about the risks that are attached to eating certain products

2. My personal situation forces me to be very knowledgeable on how I can minimize the risks of the foods I prepare

3. Given my personal situation, it is vital that I am fully up-todate on the risks of the food products I eat

4. In my situation, it is very important to be well informed on the composition of the food products I eat

8. Information dependency

1. Being well informed is very important to me

2. It is very important to me to be up-to-date

3. I feel uncomfortable when I am uninformed

4. I like to know as much as possible about topics that interest me 7-point Likert scale from

.90

1 = strongly disagree to

7 = strongly agree

7-point Likert scale from

1 = strongly disagree to

$$
7 \text { = strongly agree }
$$

7-point Likert scale from .96

1 = strongly disagree to 7 = strongly agree

7-point Likert scale from

1 = strongly disagree to 7 = strongly agree

7-point Likert scale from 1 = strongly disagree to 7 = strongly agree 
9. Information insufficiency

1. I am interested in stories in the mass media on risks of organic products

2. News reports on the influence of organically producing food on food quality do interest me

3. News items on the risks of eating organic food products interest me

4. I am interested in news stories about how I can minimize the risks of organic food products

5. I would like to know more about how I can recognize an organic product

6. I would like to learn more about the advantages and disadvantages of eating organic

7. I would like to know more about the laws on organic food producing

8. I would like to learn more about the most important differences between organic and non-organic food

10. Information seeking

I'm inclined to search for information about....

1. The disadvantages of organic products

2. The way you prepare organic products while benefitting most

3. How to prepare organic food the best

4. The way to deal best with the possible risks of eating organic

11. Trust

Food companies

1. Food companies are competent in ensuring that the food products in the Netherlands are safe to eat

2. Food companies take adequate measures to improve the consumer's health

3. When food companies claim that a food product is safe, I can rely on it

4. When food companies claim that a product is safe to eat, I can be sure of it

NVWA (Dutch Food Safety Authority)

5. The NVWA is competent in ensuring that the food products in the Netherlands are safe to eat

6. The NVWA takes adequate measures to improve the consumer's health

7. When the NVWA claims that a food product is safe, I can rely on it

8. When the NVWA claims that a product is safe to eat, I can be sure of it

Consumentenbond (Consumer organisation)

9. The 'Consumentenbond' is competent in ensuring that the food products in the Netherlands are safe to eat

10. The 'Consumentenbond' takes adequate measures to improve the consumer's health

11. When the 'Consumentenbond' claims that a food product is safe, I can rely on it

12. When the 'Consumentenbond' claims that a product is safe to eat, I can be sure of it

Retail:

13. I rely that food products that are for sale in supermarkets are adequately checked

14. I have complete confidence in the safety of the food products that are for sale

15. Food products for sale in supermarkets are safe to eat 7-point Likert scale from

1 = strongly disagree to

7 = strongly agree

.97

7-point Likert scale from

.94

1 = strongly disagree to

$7=$ strongly agree

7-point scale from $1=$ strongly disagree to

7 = strongly agree 
12. Risk perception

7-point scale from $1=$

.94

1. I think that organic food is bad for my health strongly disagree to

2. I think that there are many risks attached to organic food

7 = strongly agree

3. I think that organic food has many disadvantages

4. I think that organic food is dangerous for my health

13. Anxiety

7-point scale from $1=$

When I think about the risks of eating organic food products, I feel..

strongly disagree to

7 = strongly agree

1. Anxious

2. Worried

3. Afraid

4. Concerned 


\section{CHAPTER 6}

\section{DISCUSSION}


As a result of the development of the Internet and social media, information about food issues is in abundance available coming from a wide variety of sources, such as the traditional media, non-profit organizations and commercial enterprises. Social media also enable individuals to exchange information rapidly with others. The abundance of facts and viewpoints might be helpful as well as confusing to individuals. It requires individuals to make sense of the information and to, subsequently, decide on a course of action.

Sense-making is the process by which individuals give meaning to the world around them. It involves recognizing a problem, seeking, finding and integrating new information in a way that there is no tension between the newly encountered information and one's own vision and beliefs (Weick, 1995; Weick, Sutcliffe \& Obstfeld, 2005). Fundamental elements of the sense-making process are thus information seeking, finding, processing, using, creating, and sharing (Dervin, 1992; Pirolli and Russell, 2011; Savolainen, 1993). The collaborative sense-making perspective (Weick, 1995) focuses on group dynamics and the role of the social environment, and stresses how individuals structure unknown events to be able to act, while the representation construction model of sense-making perspective (Russell et al., 1993) and the sense-making as cognitive activity perspective (Dervin, 1983; Klein et al, 2006a, 2006b) mainly focus on individual cognitive activities needed to create meaning. How the process of sense-making is influenced by the online social environment, and social media interaction in particular, is as yet largely unknown.

The collaborative sense-making perspective has mainly been applied to sensemaking within organisations, while the other two perspectives have been used to study individual sense-making. An important question is therefore whether these sensemaking perspectives are also helpful in explaining how individuals make sense of information that is available on social media. A subsequent question is to what extent the insights in the way individuals make sense of social media-mediated information, has implications for sense-making theory. A third question is then what the consequences are for risk communication, in particular social media-mediated risk communication. This thesis aims to shed some light on these questions.

Based on the new opportunities that social media offer to (risk) communication, a distinction in three types of online information exchange that seemed most relevant in the context of risk communication is made: information exchange via social networking sites (Facebook), direct online interaction via a chat calling for a higher level of involvement, and actively sharing encountered information with others via online media such as (micro)blogs. These types of online information exchange vary in the involvement of the individual. Whereas information exchange via social networking sites relates to processing information posted by peers and other individuals or organisations in the individual's social network, online interaction via a chat calls for a 
higher level of involvement. Information sharing seems to imply an even higher level of involvement, as the individual is taking the initiative to interact with others via social media.

Organic food is chosen as the topic of this dissertation because these products are becoming more popular and available (FoodHolland, 2016; Giraud, 2002; Hughner, McDonagh, Prothero, Shultz, \& Stanton, 2007), while at the same time individuals are likely to underestimate the risks of these products. Previous studies (e.g. Hughner, 2007) mainly focused on reasons to buy organic and while there is a lot of information available regarding organic food (non-) buyers, we provide new insight in individuals' perceptions of organic food, that are central to understanding how individuals make sense of information about organic food.

The main research question of this dissertation is thus: How do individuals make sense of (online) risk information about (organic) food issues?

The main contribution of this dissertation involves insight in the role of the social environment in sense-making processes regarding organic food. We show that individuals are influenced by their social environment in the way in which they make sense of food risk information. That is, individuals' information need, taking notice of information, information seeking and information sharing behaviours are dependent on the opinions and involvement of individuals in the social environment. We find evidence that perceived similarity of others stimulates the need to make sense irrespectively of the message. In addition, we show that risk perception was influenced by the perceived expertise of the conversation partner.

The research question called for a variety of research strategies. A strong point of this dissertation is thus the mixed method approach and the variety of data analysis techniques that were used. Besides experimental designs used in the Facebook studies (chapter 3) and the online chat study (chapter 4) where we applied GLM to analyse the data, we used free response format and qualitative data analysis in the associations study (chapter 2) and conducted a survey study to examine information sharing analysed through structural equation modelling (chapter 5).

Another strong point of the research in this dissertation is that we were able to make use of representative samples, which increases the generalizability of our results to the Dutch population. This was the case in Facebook study 3.1 (chapter 3), the online chat experiment (chapter 4), and the information sharing survey (chapter 5). 


\section{Summary of the results}

\section{Associations and perceptions of organic food}

As a first step to sense-making, in the first two studies (reported in chapter 2), my co-authors and I focussed on the representation constructions of organic products. Respondents wrote down their word associations with organic food, organic meat and organic vegetables. After that, an independent sample rated these associations on centrality.

The results show that consumers associate organic food mostly with animal welfare, price, health, pesticides, and naturalness. Associations related to environment, health, honesty, pesticides, sustainability, quality, natural, additives, origin, certification, and taste were central across all food types. Associations related to meat expressed a concern for animal welfare, while associations regarding vegetables expressed a concern for human health. Consumers who according to their own account frequently bought organic food were more positive about organic food products compared to occasional buyers and individuals who hardly ever bought organic products. Consumer groups (frequently, occasional, hardly ever buyers) also differed with respect to psychological distance, values, gender and age. Based on these findings, and in line with previous studies, we conclude that organic food is viewed as quite positive, while differences between food types and consumer groups are present.

In line with Russell, Stefik, Pirolli, and Card (1993), we endorse the idea that individuals use these initial representations to encode data in order to develop more refined representations and use these representations to organize newly encountered information. These initial representations can also be viewed as the situation, e.g. the context in which sense is created (Dervin, 1983, 1992, 1998, 1999), and the framework individuals start with when making sense (Klein et al., 2006a, 2006b). The initial representations were operationalized in our successive studies as initial or preexperimental attitudes (chapter 3, chapter 4) and perceptions (chapter 5).

\section{The role of the online social environment}

In two Facebook studies (reported in chapter 3) and an online chat experiment (reported in chapter 4) we investigated the influence of online social information (social proof) on risk perception and sense-making. It was examined to what extent the social environment affects risk perception and sense-making in an online context.

Results of the first Facebook study indicate that Facebook comments are most influential when individuals perceive the comments as clear and valuable. When comment valence was either positive or negative in all the Facebook comments that the participants viewed, the number of likes on Facebook did not affect individuals' 
attitudes towards eating organic food. It thus seemed that individuals did not need additional information, e.g. the likes, when the comments were conclusive. The second Facebook study shows that subtle cues of social proof, i.e. the likes, have an effect on individuals' reactions, specifically negative emotions, and willingness to pay, when individuals watch a mixed valence Facebook page.

Taken together, the Facebook studies show that explicit online social proof, reflected in Facebook comments, is effective in stimulating sense-making when comments are evaluated as clear. They also indicate that implicit cues of social proof, such as the number of likes, are important when individuals watch a full Facebook page with mixed valence. However, when all comments express a similar viewpoint, likes are less important. This indicates that individuals use the likes as a heuristic cue to weight the comments when information from the comments is inconclusive. To further investigate this line of reasoning one might deplete consumers' cognitive resources in future works: When consumers have to process information in a heuristic way, likes might be used as a heuristic for validation, while comments might be ignored.

With respect to online social media interaction, our simulated chat (reported in chapter 4) in which we manipulated the interaction partner (expert vs peer vs anonymous interaction partner) and the message frame (gains vs losses vs uncertainty) indicated the importance of initial attitudes on risk perception of organic food and sense-making. The more positive the initial attitude, the lower the risk perception and the higher sense-making. We found that chatting with partners who were perceived to have expertise was associated with lower levels of risk perception, while chatting with partners who were perceived to be similar was associated with higher levels of information need, intention to take notice of, and search for and share information. This study thus showed that chatting with a similar other stimulates sense-making.

\section{Information sharing}

In the final study (reported in chapter 5) individuals' motives to share information online about the risks of eating organic food were studied. We found that individuals' intention to share information online about the risks of organic food was relatively low. While conversations and email were preferred as channels to share information, Facebook was considered to be the most popular social media channel to share information.

The core of our model predicting online information sharing was tested (using structural equation modelling) including the following determinants: interest within the social environment, social norm, sociability, outcome expectancies, information insufficiency, information seeking, risk perception and anxiety. Special emphasis was placed on the social determinants, because food choices are oftentimes a social process. 
In addition, risk perceptions are socially constructed (Dake, 1992) and being part of a group plays an essential role in human behaviour (Tajfel \& Turner, 1979). In relation to the social environment, previous research also stresses the importance of social norms in relation to individuals' information sharing behaviour (e.g. Lin et al., 2013; Chiu et al., 2006; Yang et al., 2013).

Testing our model, which explained $41 \%$ of variance in information sharing, showed that social norm and outcome expectancies were most important in predicting online sharing behaviour. Social norm had the strongest direct effect on information sharing, followed by outcome expectancies and information seeking. The path from information insufficiency to information sharing was insignificant, meaning that the effect of information insufficiency was indirect, through information seeking. The path from risk perception (and anxiety) to information sharing was much weaker compared to the other paths, which might be explained by the fact that individuals associate organic food with benefits rather than risks (Avitia Rodriguez et al., 2011; Hilverda et al., 2016; Hilverda et al., 2017). It also suggests that individuals perceive the risks of organic food as an impersonal risk instead of personal risk.

There were some relationships that the model did not explain: there were substantive residual correlations between social norm on the one hand, and outcome expectancies and interest within the social environment on the other. This implies that the social pressure of the environment is closely related to the expected outcomes that individuals perceive and is closely related to the interest within the social environment. There is a social aspect in outcome expectancy that may explain the residual correlation between social norm and outcome expectancies. The same reasoning goes for interest within the social environment: the social aspect of the interest within the social environment is reflected in outcome expectancy and social norms.

All together this study demonstrates the importance of social norms, outcome expectancies and information seeking in relation to one central aspect of sensemaking, specifically information sharing. The results imply that social norms not only predict information sharing, but are also closely linked to the outcome expectancies of information sharing and the interest within the social environment regarding organic food.

\section{Limitations and recommendations for future research}

One limitation of our studies relates to the participant loss between the sample that started the study and the sample that was used in the data-analysis. Both Facebook studies (chapter 3 ) and the online chat study (chapter 4) suffered from participant loss. The main reasons for this differ. 
In the online chat study, participant loss was the result of the experimental procedure and the manipulation. The data collection took place in two waves. The first wave consisted of the measurement of the initial attitude towards organic food, while the second wave contained the experiment. The strength of this procedure is that it reduces potential sensitization as a result of the pre-test. The downside is that only slightly more than half of the first wave sample also participated in the second wave. This response rate is in line with estimates ranging between $40-70 \%$ usually found in online experiments (Göritz, 2007). The second reason for participant loss in the online chat study was that participants did not correctly answer the question on the manipulation check. In line with common practice (Oppenheimer, Meyvis, \& Davidenko, 2009), these participants were excluded from analyses. The final sample and the dropouts were compared to check whether our final sample was biased. Results showed that the participant loss in the online chat study did not result in a selective sample with respect to initial attitude toward organic products, gender, and age.

In the Facebook studies (chapter 3), the situation with respect to participant loss appears more complex. The participants were shown a picture of a fictitious Facebook page and asked about the number of likes. There were two main reasons for participant loss. Some participants did not remember the number of likes, while others classified the number of likes differently than they should have according to their experimental condition. This was in particular the case for the participants in the low-likes-condition. Further exploration suggested that this may be due to individuals using their own reference frame rather than the reference frame that was presented: some students considered 15 likes on a page to be high. Another reason for failing the manipulation check might be due to the number of likes not being a relevant aspect when viewing a Facebook-page.

In line with common practice (Oppenheimer, Meyvis, \& Davidenko, 2009), we only included the participants in the analysis who correctly filled out the manipulation check, resulting in a rather small number of participants in the low-likes condition. Comparing the final sample with the drop-outs showed that there were no significant differences regarding gender and age in study 3.1. In study 3.2, students who correctly filled out the manipulation check were on average one year older than students who did not. As this is only a small difference in a largely homogeneous sample, we consider the participant loss in the Facebook studies non-selective with respect to gender and age.

An interesting question is whether removing the participants who did not correctly remember the number of likes, was the best option. One might argue that these participants were nevertheless exposed to the manipulation and might thus unconsciously have been influenced (for review see Newell \& Shanks, 2014). Our data does not provide evidence on this point. Future research might make use of an eye- 
tracker to disentangle whether participants truly do not pay attention to the subtle cues of the number of likes, or rather do pay attention to them but do not recall, because they either consider them to be less relevant in the context of a Facebook page or because their impact is more unconsciously.

Future research could explore the effects of social media-mediated interaction on individuals' perceptions, feelings and behaviour in a natural setting, e.g. social media on the Internet, to prevent participant loss. Future research could, for instance, focus on information sharing on Twitter concerning a certain topic. This type of data can be retrieved and analysed, for example, using the R-package 'TwitteR'. It could be examined which types of tweets are more likely to be retweeted (emotional content, e.g. the use of emotional words or emoticons to express oneself; see also Pfitzner, Garas, \& Schweitzer, 2012), how individuals respond to tweets from peers and authority figures. Emotional tweets coming from peers could possible more easily enhance sense-making compared to non-emotional tweets from authorities. Another option would be to examine information posted by consumers on blog webpages. This would allow researchers to examine about what kind of topics individuals share information publicly.

Besides examining natural settings, future experimental studies could focus on varying not only the message frame and the valence of the comments, but also the information provided by this conversation partner and the presentation of the arguments given in the comments (for example one-sided vs two sided messages or emotional vs rational information). This gives insight in consumers' responses to a variety of information and may shed light on what risk communication strategies might work most effectively to enhance informed decision making. In addition, it might be an option to create a simulated group chat with more than one interaction partner at the same time. To our best knowledge, online group chat studies in relation to risk perception and sense-making about food issues are not used in research, while this more closely resembles the characteristics of a real online chat. In this dissertation, we only investigated pairs and dyadic interaction, while group interaction was not examined. When investigating group interaction, two (or more) interaction partners could reinforce each other's opinion or disagree with each other. This would provide insight in collaborative sense-making (e.g. Weick, 1995). The collaborative sensemaking perspective focusses on how individuals make sense within a team or group and hereby use information from the other team members. This is partly a group process, but the outcome is on an individual level. In addition, we propose a second approach within the collaborative sense-making perspective, collective sense-making. This approach could focus on group outcomes instead of individual sense. A group chat would offer this opportunity.

This dissertation focused on organically produced foods. Research shows that consumers generally hold positive attitudes towards organic foods (Magnusson et 
al., 2001; Saba \& Messina, 2003). This was also confirmed by the association studies (chapter 2). It remains unknown to what extent our results (chapter 3, 4, 5) are applicable to other food products that are viewed as less positive, such as foods produced through nanotechnology, and to completely different domains of consumer products. Further research is needed to provide insight in the way consumers make sense of risk information about less positively evaluated consumer products and how their online social environment influences this process. One could argue that individuals might avoid information about negative topics and as a consequence would be less inclined to make sense. The question arises to what extent the social environment is able to encourage sense-making in this type of situation.

When a coherent picture is formed about consumers' responses to online information provision on food risks (including both positively and negatively evaluated products), one possibility might be to develop an intervention tool to inform consumers about food risks. The user-friendliness and effectiveness of this tool in relation to enhancing well-informed decision making can subsequently be tested in further research.

Key recommendations for future research:

1. Study the effects of online social proof and online social media interaction on risk perception and information seeking, processing and sharing in natural settings.

2. Study risk perception and information seeking, processing and sharing in experimental studies varying the presentation of the arguments in the information provided and possibly looking at interactions with multiple partners at the same time.

3. Study risk perception and information seeking, processing and sharing in relation to consumer products that are generally evaluated negatively, such as foods produced through nanotechnology.

4. Incorporate findings regarding risk perception and information seeking, processing and sharing to create effective intervention tools that can be used to inform consumers about several types of risks in times of crisis and also about chronic risks.

\section{Theoretical contribution}

Together, the findings of the studies discussed in this dissertation address both individual and social processes in relation to sense-making. One may conclude that insights in both individual and social sense-making contribute to a better understanding of individual decision making. The next question is what our results mean for sense- 
making theory specifically. To answer this question, we collected what evidence there was throughout our research on the extent that the participants were making sense. To this end, we examined to what extent information seeking and sharing took place. We further specifically looked for evidence indicating that the fundamental elements of the sense-making process (information need, seeking, finding, processing, using, creating, and sharing) were affected by a factor related to the communication of information. In the current research, these were the communicator (including experts, peers, and anonymous interaction partners), the message (frame and valence), the receiver (initial attitude, associations, values, psychological distance) and the social environment (consisting of social norms and interest within the social environment). Please see model 6.1 for a representation of the sense-making model resulting from our research.

With respect to information need regarding organic food, it was shown that 1) that the participants in the various studies expressed a need for information and 2) that information insufficiency was the main determinant of information seeking and, indirectly, of information sharing. To a lesser extent, individuals were inclined to take notice of and search for information about the risks of organic food products. The intention to share information about the risks of organic food was relatively low.

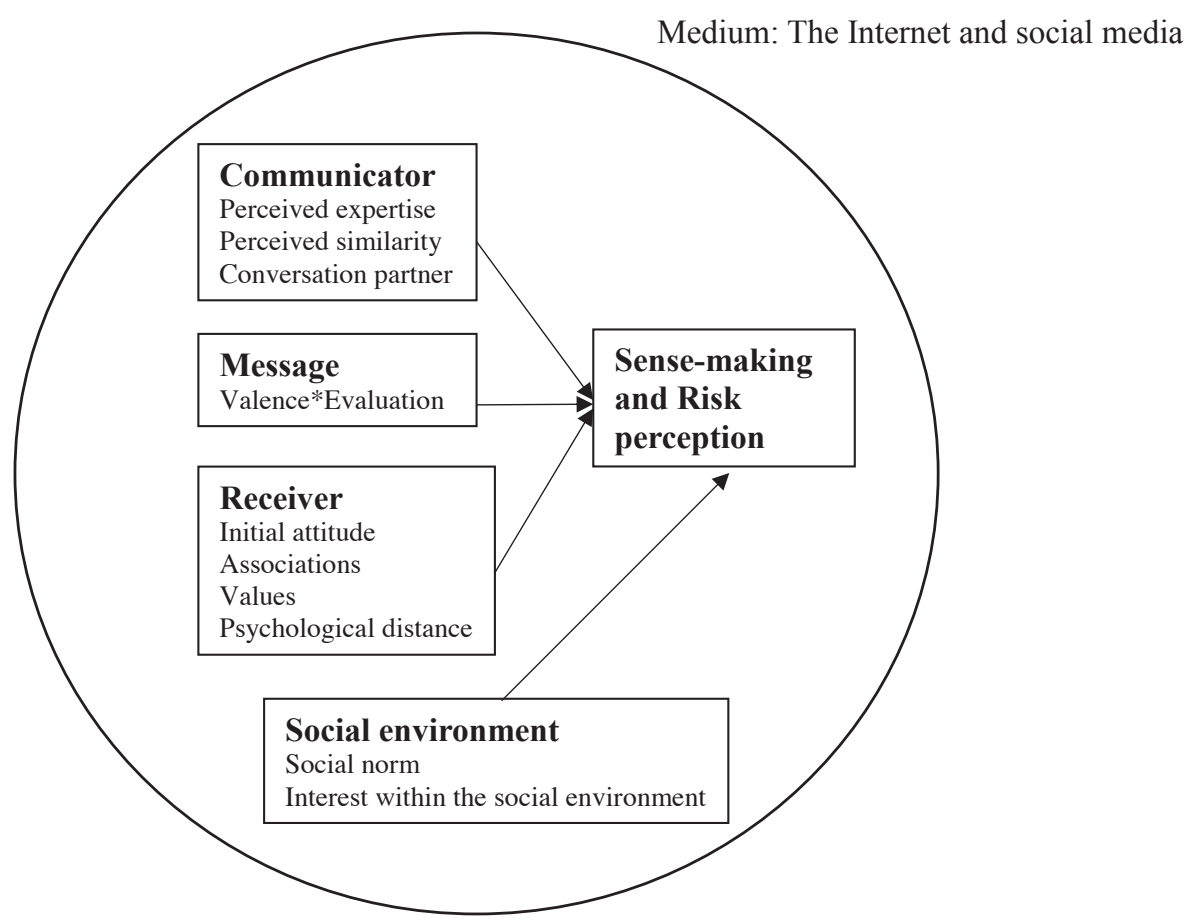

Model 6.1. Summary of the evidence for social media-mediated sense-making regarding organic foods 
Implications for sense-making are discussed looking at the fundamental aspects of communication, starting with the communicator of the message. We showed that, with respect to the communicator of the message, it is most important how this person is perceived. Being an expert resulted in a higher level of risk perception compared to being an anonymous interaction partner or a peer. Perceived expertise decreased risk perception, while perceived similarity increased sense-making. Talking with a peer can enhance sense-making irrespectively of the message frame used. We showed that not only the content of the information provided by other individuals is relevant in sensemaking, how the individual is perceived and how the information is evaluated is even more important.

Regarding the message, messages frames did not have an effect on risk perception or sense-making. The same goes for comment valence. What did matter was the interaction between comment valence and comment evaluation. This means, that when comments are perceived to be clear and useful, comment valence did have an effect, implying that sense-making only takes place when information is perceived as useful and clear.

There was also evidence that characteristics of the receiver affected sense-making. We found that the initial attitude affected risk perception and sense-making. The more positive the individual is, the lower risk perception and the higher the intention to make sense. In addition, values and psychological distance were important when differentiating consumer groups on their buying behaviour. Therefore values and psychological distance influence the outcome of sense-making, e.g. decision making regarding food purchasing, and possibly sense-making, too.

Our results showed that individuals' need to make sense is highly influenced by (information coming from) their social environment. It was shown that the (online) social environment is important in sense-making. Social norm was the strongest predictor of information sharing, while interest within the social environment largely predicted individual's information need. This means that individuals value and use the opinions of others to make sense of new information.

\section{Conclusions regarding the sense-making theory}

This dissertation contributed to the sense-making theory. Sense-making is usually approached as either an individual cognitive activity to process and understand newly encountered information, or as a collaborative process resulting in individual sense. Sense-making is often perceived as the process following a specific information gap. As far as we know, individual sense-making has not been linked to social influences processes. We show that individuals do not only make sense in collaboration with others, they are also influenced by information provided by others, and even more important how this individual is perceived. Next to the approach within the collaborative sense- 
making perspective that views individual sense as the outcome of a social process, we propose an approach, collective sense-making, in which group processes lead to group outcomes. Sense-making in an online context is influenced by the three central aspects of communication: the communicator, the message and the receiver (Pearson, Nelson, Titsworth, \& Harter, 2011).

\section{Practical recommendations}

One of the tasks of the Netherlands Food and Consumer Product Safety Authority and equivalents in other countries, is to ensure that the products that are sold in grocery stores, kitchens in restaurants or institutions, and specialty stores are safe to eat. They also inform individuals about food incidents. The results of this dissertation indicate that it might be useful for risk communicators, such as the Netherlands Food and Consumer Product Safety Authority, to communicate with consumers by using social media to facilitate well-informed decision making both during food incidents as well as to inform them about chronic risks of particular food products. One option involves allowing consumers to react to information posted on one's website. In this way, risk communicators can monitor how consumers make sense of their information and what aspects are possibly unclear to them. Another option might be to be active on social media one self, for example Twitter or Facebook, and respond to consumer comments (Veil et al, 2011). I would also advise risk communicators to incorporate an online chat tool on their website. In this way, they stimulate consumers to start a conversation with them. Such a strategy might prove to be productive as well as efficient as it corresponds to the current way consumers are interacting with each other. It would also be useful to create the opportunity for a group chat. In this way, consumers can talk with each other about food issues guided by a professional. For risk communicators, this is also useful as they can target specific consumer groups e.g. via facilitating online interaction among peers, and focus on information supply to groups of individuals rather than the individual him/herself. Next to enhancing informed decision making by using social media to communicate with individuals, using social media may possibly help authorities to build trust and a reputation of being a reliable organisation. 
Key recommendation for risk communication:

1. Recognize the importance of communicating with individuals by using social media.

2. Allow consumers to react to information posted on your website, be active on social media, and incorporate an online chat tool on your website. When using social media to communicate with individuals keep in mind that:

- information needs to be presented in a clear and useful way

- coming across as both a peer and expert is important

- $\quad$ engaging with consumers via social media might increase trust

\section{Conclusions}

Departing from a user-centred approach of sense-making, this dissertation shows how consumers make sense of food risk information concerning organic products and how this process is impacted by the online social environment. It adds to the sensemaking theory by looking at organic food as a topic and focusing on the new dynamic online information environment. Sense-making is an important topic of scientific research as it precedes decision making. Based on the evidence from this dissertation, researchers should take the online social environment into account when looking at the process of informed decision making as this environment can both influence risk perception as well as stimulate sense-making processes. 
REFERENCES 
Abraham, A., Petre, M., \& Sharp, H. (2008). Information seeking: Sensemaking and interactions. Paper presented at the CHI 2008 Workshop on Sensemaking.

Aertsens, J., Verbeke, W., Mondelaers, K., \& Van Huylenbroeck, G. (2009). Personal determinants of organic food consumption: a review. British Food Journal, 111(10), 1140-1167. doi: 10.1108/00070700910992961

Ahmad, S. N. B. B. (2010). Organic food: A study on demographic characteristics and factors influencing purchase intentions among consumers in Klang Valley, Malaysia. International journal of business and management, 5(2), 105-118.

Ajzen, I. (1991). The theory of planned behavior. Organizational behavior and human decision processes, $50(2), 179-211$.

Amblee, N., \& Bui, T. (2011). Harnessing the influence of social proof in online shopping: The effect of electronic word of mouth on sales of digital microproducts. International Journal of Electronic Commerce, 16(2), 91-114.

Andsager, J. L., Bemker, V., Choi, H.-L., \& Torwel, V. (2006). Perceived similarity of exemplar traits and behavior effects on message evaluation. Communication Research, 33(1), 3-18.

Asghar, H. M. (2015). Measuring Information Seeking through Facebook: Scale development and initial evidence of Information Seeking in Facebook Scale (ISFS). Computers in Human Behavior, 52(1), 259-270.

Attfield, S., \& Blandford, A. (2011). Making Sense of Digital Footprints in Team-Based Legal Investigations: The Acquisition of Focus. Human-Computer Interaction, 26(1-2), 38-71. doi: 10.1080/07370024.2011.556548

Avitia Rodríguez, J., Gil Roig, J. M., \& Costa Font, M. (2011). Structural equation modelling of consumer acceptance of organic food in Spain. Paper presented at the VIII Congreso de Economía Agraria.

Ayeh, J. K. (2015). Travellers' acceptance of consumer-generated media: An integrated model of technology acceptance and source credibility theories. Computers in Human Behavior, 48, 173-180. doi: http://dx.doi.org/10.1016/j.chb.2014.12.049

Baker, J. R., \& Moore, S. M. (2008). Distress, coping, and blogging: Comparing new myspace users by their intention to blog. CyberPsychology \& Behavior, 11(1), 81-85.

Bandura, A. (1997). The anatomy of stages of change. American Journal of Health Promotion, 12(1), 8-10.

Barnett, J., McConnon, A., Kennedy, J., Raats, M., Shepherd, R., Verbeke, W., . . W Wills, J. (2011). Development of strategies for effective communication of food risks and benefits across Europe: Design and conceptual framework of the FoodRisC project. BMC public health, 11(1), 308.

Basilisco, R., \& Cha, K. J. (2015). Uses and gratification motivation for using Facebook and the impact of Facebook usage on social capital and life satisfaction among Filipino users. International Journal of Software Engineering and its Applications, 9(4), 181-194.

Berger, J. (2014). Word of mouth and interpersonal communication: A review and directions for future research. Journal of Consumer Psychology, 24(4), 586-607.

Betsch, C., Ulshöfer, C., Renkewitz, F., \& Betsch, T. (2011). The influence of narrative v. statistical information on perceiving vaccination risks. Medical Decision Making, 31(5), 742-753.

Bock, G. W., \& Kim, Y.-G. (2002). Breaking the myths of rewards: An exploratory study of attitudes about knowledge sharing. Information Resources Management Journal (IRMJ), 15(2), 14 21.

Bollen, K. A. (1989). Structural equations with latent variables. Series in probability and mathematical statistics: New York, Wiley.

Bordia, P. (1997). Face-to-face versus computer-mediated communication: A synthesis of the experimental literature. Journal of Business Communication,34(1), 99-118. 
Bourn, D., \& Prescott, J. (2002). A comparison of the nutritional value, sensory qualities, and food safety of organically and conventionally produced foods. Critical reviews in food science and nutrition, 42(1), 1-34.

Bredahl, L. (1999). Consumers' Cognitions With Regard to Genetically Modified Foods. Results of a Qualitative Study in Four Countries. Appetite, 33(3), 343-360.

Bruhn, M., \& McKenzie, D. (2009). In pursuit of balance: Randomization in practice in development field experiments. American economic journal: applied economics, 1(4), 200-232.

Butcher, K. R., \& Sumner, T. (2011). Self-Directed Learning and the Sensemaking Paradox. HumanComputer Interaction, 26(1-2), 123-159. doi: 10.1080/07370024.2011.556552

Caughron, J.J., Antes, A. L., Stenmark, C. K., Thiel, C.E.,Wang,X., \& Mumford, M.D. (2013).Competition and sensemaking in ethical situations. Journal of applied social psychology, 43(7), 14911507.

CBS (2013). Bevolkingstrend 2013: Gebruik en gebruikers van sociale media. Den Haag/Heerlen: v.d. Bighelaar, S. \& Akkermans, M. G.

Cheung, C. M. K., Chiu, P.-Y., \& Lee, M. K. O. (2011). Online social networks: Why do students use facebook?" Computers in Human Behavior, 27(4), 1337-1343.

Chin, W. W. (1998). The partial least squares approach to structural equation modeling. Modern methods for business research, 295(2), 295-336.

Chiu, C. M., Hsu, M. H., \& Wang, E. T. G. (2006). Understanding knowledge sharing in virtual communities: An integration of social capital and social cognitive theories. Decision Support Systems, 42(3), 1872-1888. doi: 10.1016/j.dss.2006.04.001

Chong, D., \& Druckman, J. N. (2007). Framing theory. Annu. Rev. Polit. Sci., 10, 103-126.

Cialdini, R. B. (2001). Influence: Science and practice. Boston: Allyn \& Bacon.

Cialdini, R. B., \& Goldstein, N. J. (2004). Social influence: Compliance and conformity. Annual Review of Psychology, 55, 591-621. doi: 10.1146/annurev.psych.55.090902.142015

Clarke, C. E., \& McComas, K. (2012). Seeking and processing influenza vaccine information: A study of health care workers at a large urban hospital. Health Communication, 27(3), 244256.

Clemons, E. K., Gao, G. G., \& Hitt, L. M. (2006). When online reviews meet hyperdifferentiation: A study of the craft beer industry. Journal of Management Information Systems, 23(2), 149171.

Colville, I., Brown, A. D., \& Pye, A. (2012). Simplexity: Sensemaking, organizing and storytelling for our time. Human Relations, 65(1), 5-15. doi: 10.1177/0018726711425617

Cruwys, T., Bevelander, K. E., \& Hermans, R. C. (2015). Social modeling of eating: A review of when and why social influence affects food intake and choice. Appetite, 86, 3-18.

Dake, K. (1992). Myths of nature: Culture and the social construction of risk.Journal of Social issues, 48(4), 21-37.

Davis, A., \& Khazanchi, D. (2008). An Empirical Study of Online Word of Mouth as a Predictor for Multi-product Category e-Commerce Sales. Electronic Markets, 18(2), 130-141. doi: $10.1080 / 10196780802044776$

Dellarocas, C. (2003). The digitization of word of mouth: Promise and challenges of online feedback mechanisms. Management science, 49(10), 1407-1424.

Dervin, B. (1983). An Overview of sense-making research: Concepts, methods, and results to date. Paper presented at the International Communication Association Meeting, Dallas, TX.

Dervin, B. (1992). From the mind's eye of the user: The sense-making qualitative-quantitative methodology. Qualitative research in information management, 9, 61-84. 
Dervin, B. (1998). Sense-making theory and practice: An overview of user interests in knowledge seeking and use. Journal of Knowledge Management, 2(2), 36-46. doi: $10.1108 / 13673279810249369$

Dervin, B. (1999). On studying information seeking methodologically: the implications of connecting metatheory to method. Information Processing \& Management, 35(6), 727-750.

De Vries, R., Ashton, M., \& Lee, K. (2009). De zes belangrijkste persoonlijkheidsdimensies en de HEXACO persoonlijkheidsvragenlijst. Gedrag en Organisatie, 22(3).

Dickson-Spillmann, M., Siegrist, M., \& Keller, C. (2011). Attitudes toward chemicals are associated with preference for natural food. Food Quality and Preference, 22(1), 149-156. doi: 10.1016/j.foodqual.2010.09.001

Diehl, M. (1990). The minimal group paradigm: Theoretical explanations and empirical findings. European review of social psychology, 1(1), 263-292.

Dominguez, C., Long, W. G., Miller, T. E., \& Wiggins, S. L. (2006, June). Design directions for support of submarine commanding officer decision making. In Proceedings of 2006 Undersea HSI Symposium: Research, Acquisition and the Warrior (pp. 6-8).

Eagly, A. H., \& Chaiken, S. (1993). The psychology of attitudes. Harcourt Brace Jovanovich College Publishers.

EC Regulation Council (2007). No 834/2007 of 28 June 2007 on organic production and labelling of organic products and repealing Regulation (EEC) No 2092/91. Official Journal of the European Union, L 189, 1-23.

Eden, S. (2009). Food labels as boundary objects: How consumers make sense of organic and functional foods. Public understanding of science, 20(2), 179-194.

Eiser, J. R., Stafford, T., Henneberry, J., \& Catney, P. (2009). “Trust me, I'm a Scientist (Not a Developer)": Perceived Expertise and Motives as Predictors of Trust in Assessment of Risk from Contaminated Land. Risk Analysis, 29(2), 288-297.

Faraji-Rad, A., Samuelsen, B. M., \& Warlop, L. (2015). On the Persuasiveness of Similar Others: The Role of Mentalizing and the Feeling of Certainty. Journal of Consumer Research, 42(3), 458-471. doi: 10.1093/jcr/ucv032

Fehr, B. (1988). Prototype analysis of the concepts of love and commitment. Journal of Personality and Social Psychology, 55(4), 557-579.

Festinger, L. (1954). A theory of social comparison processes. Human Relations, 7, 114-140

Festinger, L. (1957). A theory of cognitive dissonance. Evanston, Il: Row, Peterson.

Fiedler, K. (2007). Construal level theory as an integrative framework for behavioral decisionmaking research and consumer psychology. Journal of Consumer Psychology, 17(2), 101106.

Fiske, S. T. (2009). Social beings: Core motives in social psychology: John Wiley \& Sons.

FoodHolland (2016). Dossier Biologische Voeding. Retrieved from http://www.foodholland.nl/ dossiers/biofood/home.html

Fornell, C., \& Larcker, D. F. (1981). Evaluating structural equation models with unobservable variables and measurement error. Journal of marketing research, 39-50.

Frewer, L. J., Gupta, N., George, S., Fischer, A. R. H., Giles, E. L., \& Coles, D. (2014). Consumer attitudes towards nanotechnologies applied to food production. Trends in Food Science \& Technology, 40(2), 211-225. doi: http://dx.doi.org/10.1016/j.tifs.2014.06.005

Frewer, L. J., Howard, C., \& Shepherd, R. (1998). The influence of initial attitudes on responses to communication about genetic engineering in food production. Agriculture and Human Values, 15, 15-30. 
Frewer, L. J., Howard, C., Hedderley, D., \& Shepherd, R. (1999). Reactions to information about genetic engineering: Impact of source characteristics, perceived personal relevance, and persuasiveness. Public understanding of science, 8(1), 35-50.

Galvez-Rodriguez, M. D. M., Caba-Perez, C., López-Godoy, M. (2016). Drivers of Twitter as a strategic communication tool for non-profit organisations. Internet Research, 26(5), 1052 1071.

Garretson, J. A., \& Niedrich, R. W. (2004). Spokes-characters: Creating character trust and positive brand attitudes. Journal of advertising, 33(2), 25-36.

Gaspar, R., Luís, S., Seibt, B., Lima, M. L., Marcu, A., Rutsaert, P., ... \& Barnett, J. (2016). Consumers' avoidance of information on red meat risks: information exposure effects on attitudes and perceived knowledge. Journal of Risk Research, 19(4), 533-549. doi: $10.1080 / 13669877.2014 .1003318$

Genuis, S. K. (2012). Constructing "sense" from evolving health information: A qualitative investigation of information seeking and sense making across sources. Journal of the American Society for Information Science and Technology, 63(8), 1553-1566. doi: 10.1002/ asi.22691

Giraud, G. (2002). Organic and origin-labeled food products in Europe: Labels for consumers or from producers. Ecolabels and the Greening of the Food Market. Tufts University, Boston, 41-49.

Giustini, D. (2006). How Web 2.0 is changing medicine. British Medical Journal, 333, 1283-1284.

Glass, G. V., Peckham, P. D., \& Sanders, J. R. (1972). Consequences of failure to meet assumptions underlying the fixed effects analyses of variance and covariance. Review of educational research, 42(3), 237-288.

Göritz, A. S. (2007). Using online panels in psychological research. The Oxford handbook of Internet psychology, 473-485.

Grankvist, G., \& Biel, A. (2001). The importance of beliefs and purchase criteria in the choice of eco-labeled food products. Journal of Environmental Psychology, 21(4), 405-410.

Grankvist, G., \& Biel, A. (2007). Predictors of purchase of eco-labelled food products: A panel study. Food quality and preference, 18(4), 701-708.

Grewal, R., Cote, J. A., \& Baumgartner, H. (2004). Multicollinearity and measurement error in structural equation models: Implications for theory testing. Marketing Science, 23(4), 519-529.

Griffin, R. J., Dunwoody, S., \& Neuwirth, K. (1999). Proposed model of the relationship of risk information seeking and processing to the development of preventive behaviors. Environmental Research, 80(2), S230-S245. doi: 10.1006/enrs.1998.3940

Griffin, R. J., Neuwirth, K., Giese, J., \& Dunwoody, S. (2002). Linking the heuristic-systematic model and depth of processing. Communication Research,29(6), 705-732.

Griffin, R. J., Neuwirth, K., Dunwoody, S., \& Giese, J. (2004). Information sufficiency and risk communication. Media Psychology, 6(1), 23-61.

Griffin, R. J., Yang, Z., Ter Huurne, E., Boerner, F., Ortiz, S., \& Dunwoody, S. (2008). After the flood: Anger, attribution, and the seeking of information. Science Communication, 29(3), 285315. doi: $10.1177 / 1075547007312309$

Griskevicius, V., Cialdini, R. B., \& Goldstein, N. J. (2008). Applying (and resisting) peer influence. Mit Sloan Management Review, 49(2), 84-88.

Hair, J. F., Black, W. C., Babin, B. J., Anderson, R. E., \& Tatham, R. L. (2006). Multivariate data analysis (Vol. 6): Pearson Prentice Hall Upper Saddle River, NJ.

Hammitt, J. K. (1990). Risk Perceptions and Food Choice: An Exploratory Analysis of OrganicVersus Conventional-Produce Buyers. Risk analysis, 10(3), 367-374. 
Harper, G. C., \& Makatouni, A. (2002). Consumer perception of organic food production and farm animal welfare. British Food Journal, 104(3), 287-299.

Hay, J. (1989). The consumer's perspective on organic foods. Canadian Institute of Food Science and Technology Journal, 22(2), 95-99.

He, W., \& Wei, K.-K. (2009). What drives continued knowledge sharing? An investigation of knowledge-contribution and -seeking beliefs. Decision Support Systems, 46(4), 826-838. doi: http://dx.doi.org/10.1016/j.dss.2008.11.007

Hedrick, T. E., Bickman, L., \& Rog, D. J. (1993), Applied research design: A practical guide, Sage Publications.

Helm, S. (2000). Viral marketing-establishing customer relationships by 'word-ofmouse'. Electronic markets, 10(3), 158-161.

Higgins, E. T. (1997). Beyond pleasure and pain. American psychologist, 52(12), 1280-1300.

Hilverda, F., Jurgens, M., \& Kuttschreuter, M. (2016). Consumers' associations with organic produce: A prototype analysis. British Food Journal.

Hilverda, F., Kuttschreuter, M., \& Giebels, E. (2017). Online social proof and organic food: The effect of comments and likes on facebook. Food Quality and Preference, 56, 107-118.

Horst, M., Kuttschreuter, M., Gutteling, J.M. (2007). Perceived usefulness, personal experiences, risk perception and trust as determinants of adoption of e-government services in The Netherlands. Computers in Human Behavior, 23(4), 1838-1852.

Hsu, C.-L., \& Lin, J. C.-C. (2008). Acceptance of blog usage: The roles of technology acceptance, social influence and knowledge sharing motivation. Information \& Management, 45(1), 65-74.

Hsu, M. H., Ju, T. L., Yen, C. H., \& Chang, C. M. (2007). Knowledge sharing behavior in virtual communities: The relationship between trust, self-efficacy, and outcome expectations. International Journal of Human-Computer Studies, 65(2), 153-169. doi: 10.1016/j. ijhcs.2006.09.003

Hughner, R. S., McDonagh, P., Prothero, A., Shultz, C. J., \& Stanton, J. (2007). Who are organic food consumers? A compilation and review of why people purchase organic food. Journal of consumer behaviour, 6(2-3), 94-110.

Humphreys, M., Ucbasaran, D., \& Lockett, A. (2012). Sensemaking and sensegiving stories of jazz leadership. Human Relations, 65(1), 41-62. doi: 10.1177/0018726711424320

Huurne, E., \& Gutteling, J. (2008). Information needs and risk perception as predictors of risk information seeking. Journal of Risk Research, 11(7), 847-862. doi: $10.1080 / 13669870701875750$

Huurne, E. F. J. (2008). Information Seeking in a risky world: the theoretical and empirical development of FRIS: A framework of risk information seeking. University of Twente.

Jacob, C., Mathiasen, L., \& Powell, D. (2010). Designing effective messages for microbial food safety hazards. Food Control, 21(1), 1-6.

Jin, S. V., Phua, J., \& Lee, K. M. (2015). Telling stories about breastfeeding through Facebook: The impact of user-generated content (UGC) on pro-breastfeeding attitudes. Computers in Human Behavior, 46, 6-17.

Kahl, J., Baars, T., Bugel, S., Busscher, N., Huber, M., Kusche, D., .. Z Zalecka, A. (2012). Organic food quality: A framework for concept, definition and evaluation from the european perspective. Journal of the Science of Food and Agriculture, 92(14), 2760-2765. doi: 10.1002/jsfa.5640

Kahlor, L. (2010). Prism: A planned risk information seeking model. Health Communication, 25(4), 345-356. doi: 10.1080/10410231003775172

Kahlor, L., Dunwoody, S., Griffin, R. J., \& Neuwirth, K. (2006). Seeking and processing information about impersonal risk. Science Communication, 28(2), 163-194. 
Karmarkar, U. R., \& Tormala, Z. L. (2010). Believe me, I have no idea what I'm talking about: The effects of source certainty on consumer involvement and persuasion. Journal of Consumer Research, 36(6), 1033-1049.

Kankanhalli, A., Tan, B. C., \& Wei, K.-K. (2005). Contributing knowledge to electronic knowledge repositories: An empirical investigation. Mis Quarterly, 113-143.

Kause, A., Moussaid, M. Gaissmaier, W., \& Galesic, M. (2014). When peers overrule probabilities: The interplay between narratives, uncertainty and risk perception in online environments. Paper presented at the 23rd SRA-E Conference, Istanbul, Turkey.

Kim, A. J., \& Johnson, K. K. (2016). Power of consumers using social media: Examining the influences of brand-related user-generated content on Facebook. Computers in Human Behavior, 58, 98-108.

Kirschner, P. A. (2015). Facebook as learning platform: Argumentation superhighway or dead-end street?". Computers in Human Behavior, 53, 621-625.

Klein, G., Moon, B., \& Hoffman, R. R. (2006a). Making Sense of Sensemaking 1: Alternative Perspectives. Intelligent Systems, IEEE, 21(4), 70-73. doi: 10.1109/mis.2006.75

Klein, G., Moon, B., \& Hoffman, R. R. (2006b). Making sense of sensemaking 2: A macrocognitive model. Intelligent Systems, IEEE, 21(5), 88-92.

Kline, R. B. (2005). Principles and practice of structural equation modeling. New York, NY: Guilford.

Köhler, A. R., \& Som, C. (2008). Environmental and Health Implications of Nanotechnology: Have Innovators Learned the Lessons from Past Experiences? Human and Ecological Risk Assessment: An International Journal, 14(3), 512-531. doi: 10.1080/10807030802071812

Kornelis, M., De Jonge, J., Frewer, L., \& Dagevos, H. (2007). Consumer selection of foodsafety information sources. Risk Analysis, 27(2), 327-335. doi: 10.1111/j.15396924.2007.00885.x

Kuhn, K. M. (2000). Message format and audience values: Interactive effects of uncertainty information and environmental attitudes on perceived risk. Journal of Environmental Psychology, 20(1), 41-51.

Kuttschreuter, M. (2006). Psychological determinants of reactions to food risk messages. Risk Analysis, 26(4), 1045-1057. doi: 10.1111/j.1539-6924.2006.00799.x

Kuttschreuter, M., Rutsaert, P., Hilverda, F., Regan, Á., Barnett, J., \& Verbeke, W. (2014). Seeking information about food-related risks: The contribution of social media. Food Quality and Preference, 37, 10-18.

Laurent, M. R., \& Vickers, T. J. (2009). Seeking health information online: does Wikipedia matter?. Journal of the American Medical Informatics Association,16(4), 471-479.

Lea, E., \& Worsley, T. (2005). Australians' organic food beliefs, demographics and values. British Food Journal, 107(11), 855-869.

Lee, E. J., \& Shin, S. Y. (2014). When do consumers buy online product reviews? Effects of review quality, product type, and reviewer's photo. Computers in Human Behavior, 31, 356-366.

Lee, H.-J., \& Yun, Z.-S. (2015). Consumers' perceptions of organic food attributes and cognitive and affective attitudes as determinants of their purchase intentions toward organic food. Food Quality and Preference, 39, 259-267. doi: http://dx.doi.org/10.1016/j. foodqual.2014.06.002

Lee, J., Park, D. H., \& Han, I. (2008). The effect of negative online consumer reviews on product attitude: An information processing view. Electronic commerce research and applications, 7(3), 341-352.

Lin, X., Featherman, M., \& Sarker, S. (2013). Information sharing in the context of social media: An application of the theory of reasoned action and social capital theory. Paper presented at the Proceedings of the 2013 AIS SIGHCI Workshop on HCI Research in MIS. 
Liou, D.-K., Chih, W.-H., Hsu, L.-C., \& Huang, C.-Y. (2015). Investigating information sharing behavior: The mediating roles of the desire to share information in virtual communities. Information Systems and e-Business Management, 1-30. doi: 10.1007/s10257-015-0279-2

Loewenthal, K. M. (2004). An introduction to psychological tests and scales (2nd ed.). Hove, UK: Psychology Press.

Lord, J. T. (2002). Integration is key in consumer-centric approaches. Managed care interface, 15(10), 34-37.

Maclean, M., Harvey, C., \& Chia, R. (2012). Sensemaking, storytelling and the legitimization of elite business careers. Human Relations, 65(1), 17-40. doi: 10.1177/0018726711425616

Magkos, F., Arvaniti, F., \& Zampelas, A. (2006). Organic food: Buying more safety or just peace of mind? A critical review of the literature. Critical reviews in food science and nutrition, 46(1), 23-56.

Magnusson, M. K., Arvola, A., Koivisto Hursti, U.-K., Åberg, L., \& Sjödén, P.-O. (2001). Attitudes towards organic foods among Swedish consumers. British Food Journal, 103(3), 209-227.

Makatouni, A. (2002). What Motivates Consumers to Buy Organic Food in the Uk? British Food Journal, 104(3), 345-352.

Mangold, W. G., \& Faulds, D. J. (2009). Social media: The new hybrid element of the promotion mix. Business Horizons, 52(4), 357-365. doi: http://dx.doi.org/10.1016/j.bushor.2009.03.002

Marcoulides, G. A., \& Schumacker, R. E. (2013). Advanced structural equation modeling: Issues and techniques: Psychology Press.

Mesmer-Magnus, J. R., \& DeChurch, L. A. (2009). Information sharing and team performance: a meta-analysis. Journal of Applied Psychology, 94(2), 535.

Miranda, S. M., \& Saunders, C. S. (2003). The social construction of meaning: An alternative perspective on information sharing. Information Systems Research, 14(1), 87-106. doi: 10.1287/isre.14.1.87.14765

Murdoch, J., \& Miele, M. (1999). 'Back to nature': changing 'worlds of production' in the food sector. Sociologia ruralis, 39(4), 465-483.

Narayan, B., Case, D. O., \& Edwards, S. L. (2011). The role of information avoidance in everyday-life information behaviors. Proceedings of the American Society for Information Science and Technology, 48(1), 1-9.

Näslund, L., \& Pemer, F. (2012). The appropriated language: Dominant stories as a source of organizational inertia. Human Relations, 65(1), 89-110. doi: 10.1177/0018726711424322

Newell, B. R., \& Shanks, D. R. (2014). Unconscious influences on decision making: A critical review. Behavioral and Brain Sciences, 37(01), 1-19.

Oh, S., \& Syn, S. Y. (2015). Motivations for sharing information and social support in social media: A comparative analysis of facebook, twitter, delicious, youtube, and flickr. Journal of the Association for Information Science and Technology, 66(10), 2045-2060.

Okdie, B. M., Guadagno, R. E., Petrova, P. K., \& Shreves, W. B. (2013). Social Influence Online: A Tale of Gender Differences in the Effectiveness of Authority Cues. International Journal of Interactive Communication Systems and Technologies, 3, 20-31.

Oppenheimer, D. M., Meyvis, T., \& Davidenko, N. (2009). Instructional manipulation checks: Detecting satisficing to increase statistical power. Journal of Experimental Social Psychology, 45(4), 867-872.

Osgood, C. E., P. H. Tannenbaum, and G. J. Suci. (1957). The Measurement of Meaning. Urbana, University of Illinois Press.

Padel, S., \& Foster, C. (2005). Exploring the gap between attitudes and behaviour: Understanding why consumers buy or do not buy organic food. British Food Journal, 107(8), 606-625. doi: $10.1108 / 00070700510611002$ 
Paek, H.-J., Hove, T., Jeong, H. J., \& Kim, M. (2011). Peer or expert? The persuasive impact of YouTube public service announcement producers. International Journal of Advertising, 30(1), 161-188.

Papadopoulos, T., Stamati, T., \& Nopparuch, P. (2013). Exploring the determinants of knowledge sharing via employee weblogs. International Journal of Information Management, 33(1), 133-146. doi: http://dx.doi.org/10.1016/j.ijinfomgt.2012.08.002

Paul, S. A., \& Morris, M. R. (2011). Sensemaking in Collaborative Web Search. Human-Computer Interaction, 26(1-2), 72-122. doi: 10.1080/07370024.2011.559410

Pearson, D., Henryks, J., \& Jones, H. (2011). Organic food: What we know (and do not know) about consumers. Renewable Agriculture and Food Systems, 26(2), 171-177. doi: 10.1017/ s1742170510000499

Peter, C., Rossmann, C., \& Keyling, T. (2014). Exemplification 2.0. Journal of Media Psychology, 26, 19-28.

Petrescu, D.-C., Orolan, I. G., Proorocu, M., Mihăiescu, T., Paulette, L., \& Vârban, D. (2013). Organic products: Consumption habits and perceptions. Advances in Environmental Sciences, 5(1), 1-9.

Petty, R. E., Cacioppo, J. T., \& Schumann, D. (1983). Central and peripheral routes to advertising effectiveness: The moderating role of involvement.Journal of consumer research, 10(2), 135-146.

Pfitzner, R., Garas, A., \& Schweitzer, F. (2012). Emotional Divergence Influences Information Spreading in Twitter. ICWSM, 12, 2-5.

Pirolli, P., \& Card, S. (1999). Information foraging. Psychological review, 106(4), 643.

Pirolli, P., \& Card, S. (2005). The sensemaking process and leverage points for analyst technology as identified through cognitive task analysis. Paper presented at the Proceedings of International Conference on Intelligence Analysis.

Pirolli, P., \& Russell, D. M. (2011). Introduction to this Special Issue on Sensemaking. HumanComputer Interaction, 26(1-2), 1-8. doi: 10.1080/07370024.2011.556557

Platow, M. J., Haslam, S. A., Both, A., Chew, I., Cuddon, M., Goharpey, N., . . Grace, D. M. (2005). "It's not funny if they're laughing": Self-categorization, social influence, and responses to canned laughter. Journal of Experimental Social Psychology, 41(5), 542-550. doi: 10.1016/j. jesp.2004.09.005

Pornpitakp, C. (2004). The Persuasiveness of Source Credibility: A Critical Review of Five Decades' Evidence. Journal of Applied Social Psychology, 2, 243-281.

Qu, Y., \& Furnas, G. W. (2005). Sources of structure in sensemaking. Paper presented at the CHI'05 extended abstracts on Human factors in computing systems.

Rains, S. A. (2007). The impact of anonymity on perceptions of source credibility and influence in computer-mediated group communication: A test of two competing hypotheses. Communication Research, 34(1), 100-125.

Rains, S. A., \& Scott, C. R. (2007). To Identify or Not to Identify: A Theoretical Model of Receiver Responses to Anonymous Communication. Communication Theory, 17(1), 61-91. doi: 10.1111/j.1468-2885.2007.00288.x

Raykov, T., Tomer, A., \& Nesselroade, J. R. (1991). Reporting structural equation modeling results in psychology and aging: Some proposed guidelines. Psychology and Aging, 6(4), 499-503. doi: 10.1037/0882-7974.6.4.499

Redmond, E. C., \& Griffith, C. J. (2006). Assessment of consumer food safety education provided by local authorities in the UK. British Food Journal, 108(9), 732-752.

Regan, Á., McConnon, Á., Kuttschreuter, M., Rutsaert, P., Shan, L., Pieniak, Z., . . Wall, P. (2014). The impact of communicating conflicting risk and benefit messages: An experimental study on red meat information. Food Quality and Preference, 38, 107-114. 
Roberts, J. A., Hann, I.-H., \& Slaughter, S. A. (2006). Understanding the motivations, participation, and performance of open source software developers: A longitudinal study of the apache projects. Management science, 52(7), 984-999.

Roitner-Schobesberger, B., Darnhofer, I., Somsook, S., \& Vogl, C. R. (2008). Consumer perceptions of organic foods in Bangkok, Thailand. Food Policy, 33(2), 112-121.

Rozin, P., Spranca, M., Krieger, Z., Neuhaus, R., Surillo, D., Swerdlin, A., \&Wood, K. (2004). Natural preference: Instrumental and ideational/moral motivations, and the contrast between foods and medicines. Appetite, 43, 147-154.

Russell, D. M., Stefik, M. J., Pirolli, P., \& Card, S. K. (1993). The cost structure of sensemaking. In Proceedings of the INTERACT'93 and CHI'93 conference on Human factors in computing systems (pp. 269-276). ACM.

Rutsaert, P., Barnett, J., Gaspar, R., Marcu, A., Pieniak, Z., Seibt, B., ... Verbeke, W. (2015). Beyond information seeking: Consumers' online deliberation about the risks and benefits of red meat. Food Quality and Preference, 39, 191-201. doi: http://dx.doi.org/10.1016/j. foodqual.2014.07.011

Rutsaert, P., Regan, Á., Pieniak, Z., McConnon, Á., Moss, A., Wall, P., \& Verbeke, W. (2013a). The use of social media in food risk and benefit communication. Trends in Food Science \& Technology, 30(1), 84-91.

Rutsaert, P., Pieniak, Z., Regan, Á., McConnon, Á., \& Verbeke, W. (2013b). Consumer interest in receiving information through social media about the risks of pesticide residues. Food Control, 34(2), 386-392.

Rutsaert, P., Pieniak, Z., Regan, Á., McConnon, Á., Kuttschreuter, M., Lores, M., . . . Verbeke, W. (2014). Social media as a useful tool in food risk and benefit communication? A strategic orientation approach. Food Policy, 46, 84-93.

Saba, A., \& Messina, F. (2003). Attitudes towards organic foods and risk/benefit perception associated with pesticides. Food Quality and Preference, 14(8), 637-645. doi: 10.1016/ s0950-3293(02)00188-x

Salancik, G. R., \& Pfeffer, J. (1978). Social information processing approach to job attitudes and task design. Administrative Science Quarterly, 23(2), 224-253. doi: 10.2307/2392563

Savolainen, R. (1993). The sense-making theory: Reviewing the interests of a user-centered approach to information seeking and use. Information Processing \& Management, 29(1), 13-28. doi: http://dx.doi.org/10.1016/0306-4573(93)90020-E

Savolainen, R. (2006). Information use as gap-bridging: The viewpoint of sense-making methodology. Journal of the American Society for Information Science and Technology, 57(8), 1116-1125. doi: 10.1002/asi.20400

Schifferstein, H. N. J., \& Ophuis, P. (1998). Health-related determinants of organic food consumption in the Netherlands. Food Quality and Preference, 9(3), 119-133.

Schmider, E., Ziegler, M., Danay, E., Beyer, L., \& Bühner, M. (2010). Is it really robust?. Methodology, 6(4), 147-151.

Schmidt, C.W. (1999). Safe food: An all-consuming issue. Environmental health perspectives, 107(3), 144-149.

Schwartz, S. H. (2012). An Overview of the Schwartz Theory of Basic Values. Online Readings in Psychology and Culture, 2(1), 1-20 (article 11). http://dx.doi.org/10.9707/23070919.1116

Seo, S., Almanza, B., Miao, L., \& Behnke, C. (2015). The Effect of Social Media Comments on Consumers' Responses to Food Safety Information. Journal of Foodservice Business Research, 18(2), 111-131.

Shafie, F. A., \& Rennie, D. (2012). Consumer Perceptions towards Organic Food. In M. Y. Abbas \& A. F. I. Bajunid (Eds.), Proceedings of the 1st National Conference on Environment-Behaviour Studies (Vol. 49, pp. 360-367). Amsterdam: Elsevier Science Bv. 
Shao, G. (2009). Understanding the appeal of user-generated media: a uses and gratification perspective. Internet Research, 19, 7-25.

Shi, R., Messaris, P., \& Cappella, J. N. (2014). Effects of online comments on smokers' perception of antismoking public service announcements.Journal of Computer-Mediated Communication, 19(4), 975-990.

Siegrist, M. (2000). The influence of trust and perceptions of risks and benefits on the acceptance of gene technology. Risk Analysis, 20(2),195-203. doi: 10.1111/0272-4332.202020.

Siegrist, M., \& Cvetkovich, G. (2000). Perception of Hazards: The Role of Social Trust and Knowledge. Risk Analysis, 20(5), 713-20. doi: 10.1111/0272-4332.205064.

Siegrist, M., Cousin, M.-E., Kastenholz, H., \& Wiek, A. (2007). Public acceptance of nanotechnology foods and food packaging: The influence of affect and trust. Appetite, 49(2), 459-466. doi: http://dx.doi.org/10.1016/j.appet.2007.03.002

Siegrist, M., Stampfli, N., Kastenholz, H., \& Keller, C. (2008). Perceived risks and perceived benefits of different nanotechnology foods and nanotechnology food packaging. Appetite, 51(2), 283-290. doi: 10.1016/j.appet.2008.02.020

Sjöberg, L. (2000). Perceived risk and tampering with nature. Journal of risk research, 3(4), 353367.

Sjöberg, L. (2004). Principles of risk perception applied to gene technology. EMBO reports, 5, S47-S51.

Slater, M. D., \& Rouner, D. (1996). How message evaluation and source attributes may influence credibility assessment and belief change. Journalism \& Mass Communication Quarterly, 73(4), 974-991.

Slovic, P. E. (2000). The perception of risk. Earthscan publications.

Slovic, P., Finucane, M. L., Peters, E., \& MacGregor, D. G. (2004). Risk as Analysis and Risk as Feelings: Some Thoughts about Affect, Reason, Risk, and Rationality. Risk Analysis, 24, 311-322. doi:10.1111/j.0272-4332.2004.00433.x

Soler, F., Gil, J. M., \& Sanchez, M. (2002). Consumers' acceptability of organic food in Spain: Results from an experimental auction market. British Food Journal, 104(8), 670-687.

Stephenson, J. (1997). Public health experts take aim at a moving target: foodborne infections. Journal of the American Medical Association, 277(2), 97-98.

Straus, S. G. (1996). Getting a clue: The effects of communication media and information distribution on participation and performance in computer-mediated and face-to-face groups. Small Group Research, 27 (1), 115-142.

Tabachnick, B. G., \& Fidell, L. S. (2001). Using multivariate statistics.

Tajfel, H., \& Turner, J. C. (1979). An integrative theory of intergroup conflict. The social psychology of intergroup relations, 33, 47.

Tian, Y., \& Robinson, J. D. (2008). Media use and health information seeking: An empirical test of complementarity theory. Health Communication, 23(2), 184-190.

Tidwell, M. (2009). The low-carbon diet. Audubon Magazine.

Trope, Y., \& Liberman, N. (2010). Construal-level theory of psychological distance. Psychological review, 117(2), 440-463.

Trope, Y., Liberman, N., \& Wakslak, C. (2007). Construal levels and psychological distance: Effects on representation, prediction, evaluation, and behaviour. Journal of consumer psychology, 17(2), 83-95.

Tversky, A., \& Kahneman, D. (1974). Judgement under uncertainty - Heuristics and biases. Science, 185(4157), 1124-1131. doi: 10.1126/science.185.4157.1124 
van Dijk, H., Fischer, A. R., \& Frewer, L. J. (2011). Consumer Responses to Integrated Risk-Benefit Information Associated with the Consumption of Food. Risk Analysis, 31(3), 429-439. doi: 10.1111/j.1539-6924.2010.01505.x

van Dijk, H., Fischer, A. R., de Jonge, J., Rowe, G., \& Frewer, L. J. (2012). The Impact of Balanced Risk-Benefit Information and Initial Attitudes on Post-Information Attitudes. Journal of Applied Social Psychology, 42(8), 1958-1983.

Veil, S. R., Buehner, T., \& Palenchar, M. J. (2011). A work-in-process literature review: Incorporating social media in risk and crisis communication. Journal of contingencies and crisis management, 19(2), 110-122.

Veinot, T. C. (2009). Interactive acquisition and sharing: Understanding the dynamics of hiv/ aids information networks. Journal of the American Society for Information Science and Technology, 60(11), 2313-2332.

Verbeke, W. (2005). Agriculture and the food industry in the information age. European Review of Agricultural Economics, 32(3), 347-368.

Vermeulen, I. E., \& Seegers, D. (2009). Tried and tested: The impact of online hotel reviews on consumer consideration. Tourism management, 30(1), 123-127.

Verroen, S., Gutteling, J. M., \& Vries, P. W. (2013). Enhancing Self-Protective Behavior: Efficacy Beliefs and Peer Feedback in Risk Communication. Risk analysis, 33(7), 1252-1264.

Viklund, M. J. (2003). Trust and risk perception in Western Europe: A cross-national study. Risk Analysis, 23(4):727-38. doi: 10.1111/1539-6924.00351.

Wahlström, M., Salovaara, A., Salo, L., \& Oulasvirta, A. (2011). Resolving Safety-Critical Incidents in a Rally Control Center. Human-Computer Interaction, 26(1-2), 9-37. doi: 10.1080/07370024.2011.556541

Walther, J. B., DeAndrea, D., Kim, J., \& Anthony, J. C. (2010). The influence of online comments on perceptions of antimarijuana public service announcements on YouTube. Human Communication Research, 36(4), 469-492.

Weick, K. E. (1995). Sensemaking in organizations (Vol. 3): Sage.

Weick, K. E., Sutcliffe, K. M., \& Obstfeld, D. (2005). Organizing and the process of sensemaking. Organization Science, 16(4), 409-421. doi: 10.1287/orsc.1050.013

Whittle, A., \& Mueller, F. (2012). Bankers in the dock: Moral storytelling in action. Human Relations, 65(1), 111-139. doi: 10.1177/0018726711423442

Williams, P. R. D., \& Hammitt, J. K. (2001). Perceived risks of conventional and organic produce: Pesticides, pathogens, and natural toxins. Risk Analysis, 21(2), 319-330. doi: 10.1111/02724332.212114

Wilson, E. J., \& Sherrell, D. L. (1993). Source effects in communication and persuasion research: A meta-analysis of effect size. Journal of the Academy of Marketing Science, 21(2), 101-112.

Wilson, M. J., \& Wilson, M. L. (2013). A comparison of techniques for measuring sensemaking and learning within participant-generated summaries. Journal of the American Society for Information Science and Technology, 64(2), 291-306. doi: 10.1002/asi.22758

Winter, S., Brückner, C., \& Krämer, N. C. (2015). They Came, They Liked, They Commented: Social Influence on Facebook News Channels. Cyberpsychology, Behavior, and Social Networking, 18(8), 431-436.

Winterbottom, A., Bekker, H. L., Conner, M., \& Mooney, A. (2008). Does narrative information bias individual's decision making? A systematic review.Social science \& medicine, 67(12), 2079-2088.

Yan, C. (2015). Persuading People to Eat Less Junk Food: A Cognitive Resource Match Between Attitudinal Ambivalence and Health Message Framing. Health Communication, 30(3), 251260. 
Yang, Z. J., Aloe, A. M., \& Feeley, T. H. (2014). Risk information seeking and processing model: A meta-analysis. Journal of Communication, 64(1), 20-41. doi: 10.1111/jcom.12071

Yang, Z. J., Kahlor, L. A., \& Griffin, D. J. (2013). I Share, Therefore I Am: A U.S.-China. Comparison of College Students' Motivations to Share Information About Climate Change. Human Communication Research, n/a-n/a. doi: 10.1111/hcre.12018

Zanoli, R., \& Naspetti, S. (2002). Consumer motivations in the purchase of organic food: a meansend approach. British Food Journal, 104(8), 643-653.

Zhu, H., \& Huberman, B. A. (2014). To switch or not to switch understanding social influence in online choices. American Behavioral Scientist, 0002764214527089.

Zillmann, D. (2002). Exemplification theory of media influence. Media effects: Advances in theory and research, 2, 19-41. 
SUMMARY 
This dissertation examines how individuals make sense of food risk information, focussing on organic food products as topic. As a result of the development of the Internet and social media, information about food issues is in abundance available. This abundance requires individuals to make sense of the information and to, subsequently, decide on a course of action.

Sense-making is the process by which individuals give meaning to the world around them. Itinvolves recognizing a problem, seeking, finding and integrating new information in a way that there is no tension between the newly encountered information and one's own vision and beliefs (Weick, 1995; Weick, Sutcliffe \& Obstfeld, 2005). Fundamental elements of the sense-making process are thus information seeking, finding, processing, using, creating, and sharing (Dervin, 1992; Pirolli and Russell, 2011; Savolainen, 1993). The collaborative sense-making perspective (Weick, 1995) focuses on group dynamics and the role of the social environment, and stresses how individuals structure unknown events to be able to act, while the representation construction model of sense-making perspective (Russell et al., 1993) and the sense-making as cognitive activity perspective (Dervin, 1983; Klein et al, 2006a, 2006b) mainly focus on individual cognitive activities needed to create meaning. How the process of sense-making is influenced by the online social environment, and social media interaction in particular, is as yet largely unknown. The main research question of this dissertation is thus: How do individuals make sense of (online) risk information about (organic) food issues?

Special focus is placed on the influence of the social environment and on online information exchange. Based on the new opportunities that social media offer to (risk) communication, a distinction in three types of online information exchange is made: information exchange via social networking sites (Facebook), direct online interaction via a chat calling for a higher level of involvement, and actively sharing encountered information with others via online media such as (micro)blogs. These types of online information exchange vary in the involvement of the individual.

Organic food is chosen as the topic of this dissertation because these products are becoming more popular and available (Giraud, 2002; Hughner, McDonagh, Prothero, Shultz, \& Stanton, 2007), while at the same time individuals are likely to underestimate the risks of these products. The goal of this dissertation is to gain in-depth understanding of the sense-making process of individuals with regard to organic food in an online context. A total of six empirical studies (reported in chapter 2 till 5) are performed to accomplish this goal.

In chapter 2 we report two empirical studies that are conducted to provide insight in the initial attitude that individuals have towards organic food. We examine what word associations individuals have with organic food and which are most central, how associations differ between food type and consumer group, and what 
characteristics (psychological distance, values and socio-demographics) differentiate consumer groups (based on purchasing behaviour) using Construal Level Theory as a theoretical approach (Trope \& Liberman, 2010). This research extends the scope of current consumer research about organic food and provides new insight in the word associations consumers have with eating organic.

In the first study $(\mathrm{n}=154)$ we examine the word associations that respondents write down when thinking of organic food, organic meat or organic vegetables, making a distinction between frequent, occasional, and hardly ever organic food buyers. In addition, psychological distance, values, age, gender are compared across consumer groups. In the second study it is examined how central these associations are perceived to be by an independent respondent sample $(n=52)$. Both samples are convenience samples. Results show that consumers associate organic food mostly with animal welfare, price, health, pesticides, and naturalness. Associations related to environment, health, honesty, pesticides, sustainability, quality, natural, additives, origin, certification, and taste are central across all food types. When thinking of organic meat, consumers express a concern for animal welfare, while thinking of vegetables results in a concern for human health. Consumers who frequently buy organic food seem to be more positive about organic food products compared to occasional buyers and individuals who hardly ever buy organic. Consumer groups also differ with respect to psychological distance to organic food, values, gender and age. With consumers' word associations as a starting point, we closer examine the influence of the online social environment on the sensemaking process.

In chapter 3 we focus on the influence of the social environment on sense-making. As social media offer new possibilities regarding information communication, it is decided to firstly focus on responses to information on social networking sites. To get an idea of how individuals are influenced by risk and benefit information that they encounter on social networking sites, specifically Facebook, two studies are performed. In the first study, using a representative sample $(\mathrm{n}=124)$, comment valence (positive vs negative) and likes (high vs low) are manipulated. In addition, the evaluation of the comments in terms of clearness and usefulness is included as a moderator. Results indicate that explicit online social proof, such as the valence of Facebook comments, was effective in stimulating sense-making when comments are evaluated as clear. In the second study (student sample; $\mathrm{n}=88$ ), in which only the number of like are manipulated on a more realistic Facebook page, it is also shown that implicit cues of social proof, such as the number of likes, are important when individuals watch a full Facebook page with mixed valence comments. However, when valence is the same across all comments, likes are less important. 
In chapter 4 my co-authors and I want to go one step beyond social networking sites and aim to tap into online sense-making and risk perception by letting participants evaluate the risks associated with organic food products in collaboration with virtual others (peer versus experts versus anonymous author). In this study, online information exchange is operationalized as an online chat simulation. We examine whether 1) the message frame, 2) the conversation partner; his/her perceived similarity and expertise, and 3) the individual's pre-experimental attitudes affect how individuals deal with food risk and benefit information in an online communication setting.

The simulated chat study (representative sample; $\mathrm{n}=310$ ), in which we manipulate the conversation partner (expert vs peer vs anonymous) and the message frame (gains vs losses vs uncertain), shows the importance of pre-experimental attitudes on risk perception of organic food and sense-making. The more positive the initial attitude, the higher the need to make sense. Talking with an expert leads to higher levels of risk perception compared to chatting with an anonymous source of peer. It is also important how the conversation partner is perceived. Partners who are perceived to be an expert are associated with lower levels of risk perception, partners who are perceived to be similar are associated with higher levels of information need, intention to take notice, and search for and share information. The message frame does not have an effect on risk perception and sense-making.

In chapter 5 the third new opportunity of social media, information sharing, is examined more closely. An online survey among a representative sample of 535 respondents is conducted to examine the determinants information sharing behaviour, and their relationships. Structural equation modelling is applied to test both the measurement model and the structural model.

The core of our model predicting online information sharing is tested including the following determinants: interest within the social environment, social norm, sociability, outcome expectancies, information insufficiency, information seeking, risk perception and anxiety. Testing our model, which explains $41 \%$ of variance in information sharing, shows that social norm and outcome expectancies are most important in predicting online sharing behaviour. Social norm has the strongest direct effect on information sharing, followed by outcome expectancies and information seeking. The path from information insufficiency to information sharing is insignificant, meaning that the effect of information insufficiency is indirect, through information seeking. The path from risk perception (and anxiety) to information sharing is much weaker compared to the other paths.

Results also show that there are substantive residual correlations between social norm on the one hand, and outcome expectancies and interest within the social environment on the other, implying that the social pressure of the environment is 
closely related to the expected outcomes that individuals perceive and is closely related to the interest within the social environment.

In the final chapter, chapter $\mathbf{6}$, the results from previous chapters are discussed, recommendation for future research and theoretical and practical implications are provided. We can conclude that the social environment pays an important role in sensemaking about organic food risks in an online context. Next to the approach within the collaborative sense-making perspective that views individual sense as the outcome of a social process, we propose an approach, collective sense-making, in which group processes lead to group outcomes.

Our results suggest that social media can be an effective tool for risk communicators to affect risk perception and increase sense-making. An option for risk communicators would be to allow individuals to react to information posted on one's website. Another option would be to be active on social media one self, respond to consumer comments, and even try to start conversations themselves, e.g. when one would like to increase consumer awareness of risks attached to particular foods. Such a strategy might prove to be productive as well as efficient as it corresponds to the current way individuals are interacting with each other. Future research should focus on the possibility of developing an online chat tool to inform individuals about food risks, both chronic risks as well as incidents. 
SAMENVATTING 
In dit proefschrift wordt gekeken op welke manier individuen betekenis verlenen (sense-making) aan risico-informatie over voeding, met als onderwerp biologische producten. Als gevolg van de ontwikkeling van het Internet en sociale media is er een grote hoeveelheid informatie over voedingskwesties beschikbaar. Om in staat te zijn een beslissing te nemen over voedselaankopen, is het belangrijk dat individuen deze informatie verwerken en hier betekenis aan verlenen.

Sense-making is het proces waarin individuen betekenis verlenen aan de wereld om hen heen. Dit proces omvat het herkennen van een probleem, en het zoeken, vinden en integreren van nieuwe informatie op een manier waardoor deze informatie goed past bij de visie en ideeën van een persoon (Weick, 1995; Weick, Sutcliffe \& Obstfeld, 2005). Fundamentele elementen van sense-making zijn informatie zoeken, vinden, verwerken, gebruiken, creëren en delen (Dervin, 1992; Pirolli and Russell, 2011; Savolainen, 1993). Er zijn drie sense-making perspectieven te onderscheiden. Het collaborative sensemaking perspectief (Weick, 1995) richt zich op groepsdynamiek, de rol van de sociale omgeving en de wijze waarop individuen onbekende gebeurtenissen structureren voordat ze handelen. Het representation construction model of sense-making perspectief (Russell et al., 1993) en het sense-making as cognitive activity perspectief (Dervin, 1983; Klein et al, 2006a, 2006b) richten zich allebei vooral op individuele cognitieve processen die nodig zijn voor betekenisverlening. Hoe het proces van betekenisverlening beïnvloed wordt door de online sociale omgeving en interactie via sociale media in het bijzonder, is nog grotendeels onbekend. De hoofdvraag van dit proefschrift luidt dan ook: Hoe verlenen individuen betekenis aan (online) risico-informatie over (biologische) voeding?

De nadruk wordt gelegd op de invloed van de sociale omgeving en op online informatie-uitwisseling. Op basis van de nieuwe mogelijkheden van sociale media voor risico-communicatie, maken we onderscheid in drie vormen van informatieuitwisseling. Als eerste kijken we naar informatie uitwisselen via sociale netwerksites zoals Facebook. De tweede vorm die we onderzoeken is directe online interactie door middel van een chat waarbij er meer betrokkenheid vereist is. Als derde kijken we naar het actief online delen van informatie die men tegenkomt via sociale media, zoals microblogs. Deze drie vormen van informatie-uitwisseling verschillen in de benodigde betrokkenheid van het individu.

Biologische producten zijn gekozen als onderwerp van dit proefschrift, omdat deze producten steeds bekender worden en ruim beschikbaar zijn (Giraud, 2002; Hughner, McDonagh, Prothero, Shultz, \& Stanton, 2007), terwijl het tegelijkertijd waarschijnlijk is dat individuen de risico's van deze producten onderschatten. Het doel van dit proefschrift is om meer inzicht te krijgen in het proces van betekenisverlening met betrekking tot biologische producten in een online context. Hiertoe zijn zes empirische studies (beschreven in hoofdstuk $2 \mathrm{t} / \mathrm{m}$ 5) uitgevoerd. 
In hoofdstuk 2 rapporteren we twee empirische studies die inzicht geven in de initiële attitude die individuen hebben ten opzichte van biologische producten. We onderzoeken met welke woorden consumenten "biologisch" associëren, welke associaties het meest centraal zijn, en hoe de associaties verschillen tussen consumentengroepen (gebaseerd op hun koopgedrag). Ook kijken we in hoeverre consumentengroepen verschillen qua psychologische afstand, waarden en socio-demografische gegevens. We gebruiken hierbij de Construal Level Theory als theoretische invalshoek (Trope \& Liberman, 2010). Dit onderzoek verbreedt de scope van consumentenonderzoek betreffende biologische producten en geeft inzicht in met welke woorden consumenten "biologisch" associëren.

In de eerste studie $(n=154)$ kijken we naar de woorden die respondenten opschrijven als ze aan biologische voeding, biologisch vlees of biologische groenten denken. Hierbij maken we onderscheid tussen consumenten die vaak, soms, of bijna nooit biologische producten kopen. Verder worden psychologische afstand, waarden, leeftijd en geslacht bekeken voor de verschillende consumentengroepen.

In de tweede studie wordt onderzocht hoe centraal de associaties uit de eerste studie door een onafhankelijk steekproef $(n=52)$ worden beschouwd. Beide steekproeven zijn gelegenheidssteekproeven. De resultaten laten zien dat consumenten biologische voeding voornamelijk associëren met dierenwelzijn, prijs, gezondheid, natuurlijk en pesticides. Woorden die te maken hebben met het milieu, gezondheid, eerlijkheid, pesticides, duurzaamheid, kwaliteit, natuurlijk, toevoegingen, oorsprong, keurmerken en smaak zijn centraal voor alle voedingstypen.

De woorden die respondenten opschrijven bij biologisch vlees laten zien dat consumenten dierenwelzijn belangrijk vinden, terwijl de associaties bij biologische groenten het belang van gezondheid laten zien. Consumenten die vaak biologische producten kopen lijken positiever over biologische voeding dan consumenten die soms of bijna nooit dergelijke producten kopen. De consumentengroepen verschillen ook op psychologische afstand, waarden, geslacht en leeftijd. Deze associaties gebruiken we als beginpunt bij het onderzoek naar de invloed van de online sociale omgeving op het proces van betekenisverlening.

In hoofdstuk 3 ligt de focus op de invloed van de sociale omgeving op betekenisverlening. Omdat sociale media nieuwe mogelijkheden bieden met betrekking tot het communiceren van informatie, is besloten om eerst te focussen op de vraag op welke wijze mensen reageren op informatie op sociale netwerksites. Om een idee te krijgen van de manier waarop individuen reageren op risico-informatie en informatie over de voordelen van biologische producten die ze op sociale netwerksites tegenkomen, met Facebook in het bijzonder, worden twee studies uitgevoerd.

In de eerste studie, waarbij gebruik wordt gemaakt van een representatieve steekproef $(\mathrm{n}=124)$, worden de valence van opmerkingen op Facebook (positief vs 
negatief) en het aantal likes (veel vs weinig) gemanipuleerd. Verder wordt de evaluatie van de opmerkingen in termen van duidelijkheid en bruikbaarheid toegevoegd als moderator in de analyses. De resultaten laten zien dat expliciete vormen van social proof, zoals de valence van Facebookopmerkingen, betekenisverlening stimuleert wanneer de opmerkingen als duidelijk worden ervaren. In de tweede studie (studentensteekproef; $\mathrm{n}=88$ ), waarin alleen het aantal likes op een meer realistische Facebookpagina wordt gemanipuleerd, laten we zien dat impliciete vormen van social proof, zoals het aantal likes, belangrijk zijn wanneer individuen een complete Facebookpagina zien met zowel negatieve als positieve opmerkingen. Wanneer valence van alle opmerkingen hetzelfde is, echter, zijn likes minder belangrijk.

In hoofdstuk 4 gaan mijn co-auteurs en ik een stap verder dan sociale netwerksites en proberen we om online betekenisverlening te bestuderen door proefpersonen de risico's van biologische producten in samenwerking met een virtuele ander (gelijke vs expert vs anonieme bron) te laten beoordelen. In deze studie wordt online informatieuitwisseling geoperationaliseerd met behulp van een online chatsimulatie. We onderzoeken of 1) de framing van de boodschap, 2) de gesprekspartner, zijn/haar waargenomen gelijkwaardigheid en expertise, en 3) de initiële attitude van het individu effect hebben op de manier waarop individuen omgaan met voedingsrisico-informatie en informatie over de voordelen van biologische producten in een online setting.

De gesimuleerde chatstudie (representatieve steekproef; $n=310$ ), waarin we de gesprekspartner (gelijke vs expert vs anonieme bron) en de framing van de boodschap (positief vs negatief vs onzeker) manipuleren, laat zien dat de initiële attitude belangrijk is. Hoe positiever deze attitude, des te lager risicoperceptie en des te hoger de behoefte aan betekenisverlening. Praten met een expert leidt tot een hoger niveau van risicoperceptie vergeleken met praten met een anonieme bron of een gelijke ander. Het is ook belangrijk hoe de gesprekspartner wordt waargenomen. Hoe meer de partners worden waargenomen als experts, des te lager de risicoperceptie. Verder laten de resultaten zien dat hoe meer de partners worden waargenomen als gelijke ander, des te hoger de mate van informatiebehoefte, intentie om te letten op informatie, informatie te zoeken en informatie te delen. Het frame van de boodschap heeft geen effect op risicoperceptie en betekenisverlening.

In hoofdstuk 5 wordt informatie delen onderzocht. Wij zien dit als de derde vorm van informatie-uitwisseling via sociale media, waarbij het individu informatie actief deelt met anderen. Een online survey met een representatieve steekproef van 535 respondenten is uitgevoerd om te onderzoeken wat de determinanten van online informatie delen zijn en welke onderlinge relaties tussen deze determinanten aanwezig zijn. Structural equation modelling wordt gebruikt om zowel het meetmodel als het structurele model te toetsen. 
De kern van het model, dat online informatie delen voorspelt, wordt getoetst met de volgende determinanten: interesse binnen de sociale omgeving, sociale norm, sociabiliteit, de uitkomsten die men als gevolg van het delen van informatie met anderen worden verwacht, informatiebehoefte (information sufficiency), informatie zoeken, risicoperceptie en angst. Deze determinanten verklaren samen $41 \%$ van de variantie van informatie delen. De resultaten laten verder zien dat sociale norm en de verwachte uitkomsten de belangrijkste voorspellers zijn van online informatie delen. Sociale norm heeft het sterkste directe effect op informatie delen, gevolgd door de verwachte uitkomsten en informatie zoeken. Het pad van informatiebehoefte naar informatie delen is niet significant. Dit betekent dat het effect van informatiebehoefte indirect is en via informatie zoeken loopt. Het pad van risicoperceptie (en angst) naar informatie delen is veel zwakker dan de andere paden.

De resultaten laten ook zien dat er aanzienlijke residuele correlaties zijn tussen sociale norm aan de ene kant en de verwachte uitkomsten en de interesse in de sociale omgeving aan de andere kant. Dit impliceert dat de sociale druk van de omgeving sterk gerelateerd is aan de verwachte uitkomsten van informatie delen en sterk samenhangt met de interesse van de sociale omgeving.

In het laatste hoofdstuk, hoofdstuk 6, worden de resultaten van de voorgaande hoofdstukken besproken, worden aanbevelingen voor vervolgonderzoek gedaan en worden theoretische en praktische implicaties gegeven. We kunnen concluderen dat de sociale omgeving een belangrijke rol speelt in het betekenis verlenen aan informatie over de risico's van biologische producten in een online context. Naast een benadering binnen het collaborative sense-making perspectief waarin individuele betekenis wordt gezien als de uitkomst van een sociaal proces, stellen wij een collective sense-making benadering voor, waarin groepsprocessen tot groepsuitkomsten leiden.

Onze resultaten suggereren dat sociale media een effectieve tool kunnen zijn voor organisaties als de NVWA die communiceren over voedingsrisico's, om risicoperceptie te beïnvloeden en betekenisverlening te stimuleren. Een optie zou zijn om individuen de mogelijkheid te geven om te reageren op informatie op hun website. Een andere optie zou kunnen zijn om zelf actief te zijn op sociale media en te reageren op reacties van consumenten, en zelf te proberen met consumenten in gesprek te komen, om zo het bewustzijn van de risico's van bepaalde voedingsmiddelen te vergroten. Zo'n strategie kan vruchtbaar en efficiënt zijn, omdat deze aansluit bij de manier waarop individuen hedendaags met elkaar communiceren. Vervolgonderzoek zou zich moeten richten op de mogelijkheid een online chat tool te ontwikkelen om individuen te informeren over voedingsrisico's, zowel chronische risico's als incidenten. 


\section{DANKWOORD}


Promoveren was voor mij net als sense-making een proces dat naast een individuele activiteit ook vele sociale aspecten kende en beïnvloed werd door mijn sociale omgeving. Veel mensen uit mijn sociale omgeving hebben, direct of indirect, een bijdrage geleverd aan dit proefschrift. Ik wil dan ook iedereen die heeft bijgedragen bedanken. Graag wil ik een aantal mensen in het bijzonder noemen.

Ten eerste wil ik graag mijn dagelijkse begeleider, Margot, bedanken. Jouw kritische feedback en onze gesprekken hielpen me het proefschrift naar een hoger niveau te brengen. Daarnaast wil ik mijn promotor, Ellen, bedanken. Jouw helikopter-view hielp me om het grote geheel te zien. Ook gaat mijn dank uit naar de leden van de promotiecommissie die hun tijd en energie hebben gestoken in het doornemen en beoordelen van mijn proefschrift.

Ik wil graag een aantal (oud-)studenten bedanken die me hebben ondersteund bij het uitvoeren van mijn onderzoeken. Manon Jurgens, Daniela Guddorp, Lilian Hofland, Sarah Reinartz en Christina Reker, bedankt voor jullie hulp bij het ontwikkelen van de materialen voor mijn onderzoeken en/of het helpen met dataverzameling.

Tijdens mijn promotie heb ik een erg prettige tijd gehad met mijn collega's bij PCRV. Hoewel ik met alle collega's en vooral mijn mede-promovendi leuke en leerzame momenten heb meegemaakt, wil ik graag een paar collega's in het bijzonder noemen. Saskia en Anja, mijn tijd bij de vakgroep ben ik met jullie samen begonnen. Jullie hebben me wegwijs gemaakt bij PCRV en in Enschede. Ik ben dankbaar voor de waardevolle vriendschap die we hebben opgebouwd. Marian en Miriam de Graaff, wij hebben een groot deel van onze promoties samen doorgebracht. Ik wil jullie bedanken voor jullie input en support tijdens deze periode! Marije en Wendy, lieve paranimfen, kantoorgenoten, vriendinnen, sportmaatjes, ik ben ontzettend blij met jullie! Naast onze inhoudelijke discussies over onderzoek en onderwijs, heb ik ook veel steun aan jullie door de sociale activiteiten die we samen ondernemen, zoals het sporten, lekker eten bij het Foodtruck festival en La Cubanita.

Naast begeleiders, commissie en collega's wil ik graag ook iedereen bedanken die me heeft geïnspireerd tijdens congresbijeenkomsten van het SRA-E en cursussen van het KLI.

Ook wil ik hier graag Inga bedanken voor het ontwerpen van mijn cover. Verder wil ik haar en Sabine bedanken voor de gezellige "pasta en wijn"- avonden die we samen hebben gehad tijdens onze promotietrajecten. 
Daarnaast wil ik Muirne bedanken voor onze inspirerende gesprekken over onderzoek en ons intensieve contact op de UT en daarbuiten. We hebben tijdens mijn promotie een hechte vriendschap opgebouwd. Ook wil ik hier Maarten bedanken. Je hebt mij tijdens onze telefoongesprekken geholpen om alles te relativeren en positief te blijven denken.

Verder wil ik graag een aantal belangrijke personen in mijn leven bedanken. Guusje, Hadewych en Renée, jullie zijn al jaren mijn goede vriendinnen. Jullie hebben mij geholpen te ontspannen en zo nu en dan mijn proefschrift te 'vergeten', zodat ik later weer met een frisse blik verder kon. Ook wil ik mijn grote broers, Dirk en Jannes, en mijn "lievelingszusje", Lotte, bedanken. Jullie zijn er altijd voor me en ondanks dat we alle vier heel verschillende "kinderen" zijn, ben ik blij dat we elkaar helpen en steunen als dat nodig is. Lieve Tobias, ik ben ontzettend blij dat wij elkaar hebben leren kennen en dat je mij in alles wat ik doe steunt. Jouw goede zorg hielp me door de laatste drukke maanden heen. Als laatste, Lieve papa en mama, jullie zijn er altijd voor me! Dank voor jullie onvoorwaardelijke steun en liefde. 
KLI DISSERTATION SERIES 
The "Kurt Lewin Institute Dissertation Series" started in 1997. Since 2014 the following dissertations have been published in this series:

2014-01: $\quad$ Marijn Stok: Eating by the Norm: The Influence of Social Norms on Young People's Eating Behavior

2014-02: $\quad$ Michèlle Bal: Making Sense of Injustice: Benign and Derogatory Reactions to Innocent Victims

2014-03: Nicoletta Dimitrova: Rethinking errors: How error-handling strategy affects our thoughts and others' thoughts about us

2014-04: $\quad$ Namkje Koudenburg: Conversational Flow: The Emergence and Regulation of Solidarity through social interaction

2014-05: Thomas Sitser: Predicting sales performance: Strengthening the personality - job performance linkage

2014-06: Goda Perlaviciute: Goal-driven evaluations of sustainable products

2014-07: Said Shafa: In the eyes of others: The role of honor concerns in explaining and preventing insult-elicited aggression

2014-08: $\quad$ Felice van Nunspeet: Neural correlates of the motivation to be moral

2014-09: Anne Fetsje Sluis: Towards a virtuous society: Virtues as potential instruments to enhance

2014-10: Gerdien de Vries: Pitfalls in the Communication about CO2 Capture and Storage

2014-11: Thecla Brakel: The effects of social comparison information on cancer survivors' quality of life: A field-experimental intervention approach

2014-12: Hans Marien: Understanding and Motivating Human Control: Outcome and Reward Information in Action

2014-13: $\quad$ Daniel Alink: Public Trust: Expectancies, Beliefs, and Behavior

2014-14: $\quad$ Linda Daphne Muusses: How Internet use may affect our relationships: Characteristics of Internet use and personal and relational wellbeing

2014-15: Hillie Aaldering: Parochial and universal cooperation in intergroup conflicts

2014-16: $\quad$ Martijn Keizer: Do norms matter? The role of normative considerations as predictors of pro-environmental behavior

2015-01: $\quad$ Maartje Elshout: Vengeance

2015-02: $\quad$ Seval Gündemir: The Minority Glass Ceiling Hypothesis: Exploring Reasons and Remedies for the Underrepresentation of Racial-ethnic Minorities in Leadership Positions

2015-03: Dagmar Beudeker: On regulatory focus and performance in organizational environments 
2015-04: Charlotte Koot: Making up your mind about a complex technology: An investigation into factors that help or hinder the achievement of cognitive closure about CCS

2015-05: $\quad$ Marco van Bommel: The Reputable Bystander: The Role of Reputation in Activating or Deactivating Bystanders

2015-06: $\quad$ Kira 0. McCabe: The Role of Personality in the Pursuit of Context-Specific Goals

2015-07: Wiebren Jansen: Social inclusion in diverse work settings

2015-08: Xiaoqian Li: As time goes by: Studies on the subjective perception of the speed by which time passes

2015-09: Aukje Verhoeven: Facilitating food-related planning. Applying metacognition, cue-monitoring, and implementation intentions

2015-10: Jasper de Groot: Chemosignaling Emotions: What a Smell can Tell

2015-11: Hedy Greijdanus: Intragroup Communication in Intergroup Conflict: Influences on Social Perception and Cognition

2015-12: Bart de Vos: Communicating Anger and Contempt in Intergroup Conflict: Exploring their Relational Functions

2015-13: Gerdientje Danner: Psychological Availability. How work experiences spill over into daily family interactions

2015-14: Hannah Nohlen: Solving ambivalence in context. The experience and resolution of attitudinal ambivalence

2015-15: $\quad$ Stacey Sanders: Unearthing the Moral Emotive Compass: Exploring the Paths to (Un)Ethical Leadership

2015-16: Marc Heerdink: Regulating deviance with emotions: Emotional expressions as signals of acceptance and rejection

2015-17: Danny Taufik: "Can you feel it" The role of feelings in explaining proenvironmental behavior

2015-18: Sarah Elbert: Auditory information and its parameters in health persuasion. The development of a tailored smartphone application to support behavior change

2016-01: Anna van 't Veer: Effortless morality - cognitive and affective processes in deception and its detection

2016-02: Thijs Bouman: Threat by association: How distant events can affect local intergroup relations

2016-03: Tim Theeboom: Workplace coaching: Processes and effects

2016-04: Sabine Strofer: Deceptive intent: Physiological reactions in different interpersonal contexts 
2016-05: Caspar van Lissa: Exercising Empathy: The Role of Adolescents' Developing Empathy in Conflicts with Parents

2016-06: Marlon Mooijman: On the determinants and consequences of punishment goals: The role of power, distrust, and rule compliance

2016-07: Niels van Doesum: Social mindfulness

2016-08: Leonie Venhoeven: A look on the bright side of an environmentally-friendly life: Whether and why acting environmentally-friendly can contribute to well-being

2016-09: $\quad$ Florien Cramwinckel: The social dynamics of morality

2016-10: Junhui Wu: Understanding Human Cooperation: The Psychology of Gossip, Reputation, and Life History

2016-11: $\quad$ Elise C. Seip: Desire for vengeance. An emotion-based approach to revenge

2016-12: Welmer E. Molenmaker: The (un)willingness to reward cooperation and punish non-cooperation

2016-13: Liesbeth Mann: On Feeling Humiliated. The Experience of Humiliation in Interpersonal, Intragroup, and Intergroup Contexts

2016-14: $\quad$ Angela M. Ruepert: Working on the environment

2016-15: Femke Hilverda: Making sense of food risk information: The case of organic food 


\section{Making Sense of Food Risk Information The Case of Organic Food}

When individuals encounter new information about food issues, such as organic food risks, they have to make sense of this information. Sense-making is the process by which individuals give meaning to the world around them. How the process of sense-making is influenced by the online social environment, and social media interaction in particular, is as yet largely unknown. This dissertation therefore examines the research question: How do individuals make sense of (online) risk information about (organic) food issues? Special focus is placed on the influence of the social environment and on online information exchange. Based on the new opportunities that social media offer to (risk) communication, a distinction in three types of online information exchange is made: information exchange via social networking sites (Facebook), direct online interaction via a chat, and actively sharing encountered information with others via online media such as (micro)blogs. A total of six empirical studies are performed to provide insight in sense-making regarding organic food risks in an online context.

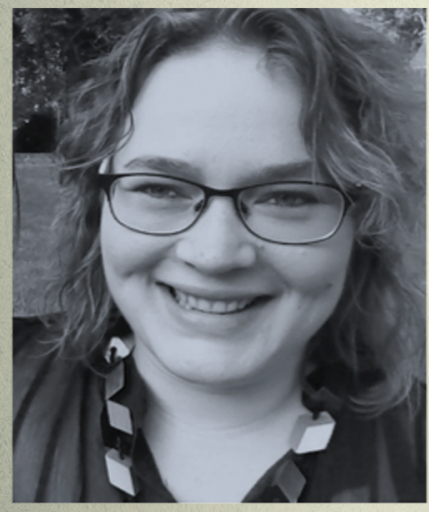

Femke Hilverda is currently a lecturer at the Work and Organisational Psychology department at Radboud University Nijmegen. She also works as a lecturer at the Faculty of Behavioural, Management and Social Sciences at the University of Twente. Her PhD research, completed at the Department of Psychology of Conflict, Risk and Safety at the University of Twente, combines social psychology and communication research.

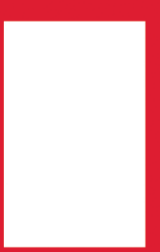

k u r t I e Dissertation Series

Kurt Lewin Institute

w i n i n s

2016-15 